Florida International University FIU Digital Commons

$12-7-1996$

\title{
Parasexuality and heterokaryosis in fusarium oxysporum forma specialis cubense
}

Blanca R. Cortes

Florida International University

DOI: $10.25148 /$ etd.FI14061524

Follow this and additional works at: https://digitalcommons.fiu.edu/etd

Part of the Biology Commons

\section{Recommended Citation}

Cortes, Blanca R., "Parasexuality and heterokaryosis in fusarium oxysporum forma specialis cubense" (1996). FIU Electronic Theses and Dissertations. 2717.

https://digitalcommons.fiu.edu/etd/2717 
FLORIDA INTERNATIONAL UNIVERSITY

Miami, Florida

PARASEXUALITY AND HETEROKARYOSIS IN FUSARIUM OXYSPORUM FORMA SPECIALIS CUBENSE

A thesis submitted in partial satisfaction of the requirements for the degree of MASTER OF SCIENCE

IN

BIOLOGY

by

Blanca R. Cortes

1996 
To: Dean Arthur W. Herriott

College of Arts and Sciences

This thesis, written by Blanca $R$, Cortes, and entitled

Parasexuality and Heterokaryosis in Fusarium oxysporum forma specialis cubense, having been approved in respect to style and intellectual content, is referred to you for judgement.

We have read this thesis and recommend that it be approved.

Jeff Elhai

John Makemson

David N. Kuhn, Major Professor

Date of Defense: December 7, 1995

The thesis of Blanca $R$. Cortes is approved.

Dean Arthur W. Herriott

College of Arts and Sciences

Dr. Richard L. Campbell

Dean of Graduate studies

Florida International University, 1996 
I dedicate this thesis to my parents and family for their patience and support. A special dedication goes to grandma... 


\section{ACKNOWLEDGMENTS}

I wish to thank the members of my committee for their helpful comments and encouragement to exploit the resources available to me. I also want to thank the members of the lab for their assistance, Theresa Pinto-Sherer, Wilber Quintanilla, Giovanni Recalde, Hugo Fonseca and Suzy Hector. Special thanks to Chris sinigalliano for his useful tips in computer operations and advise, and to stacey west and her staff for the wonderful slides and graphic tools used on my presentations. I would also like to thank the Minority Biomedical Research Support Program for their support.

Last, but not least, I would like to thank my major professor, Dr. Kuhn, for his support, patience and guidance. His foresight to work always with a plan B has helped me not only toward the completion of this project but in my personal life as well. He was always there for me through the ups and downs of the research and encouraged me to continue trying when things didn't go my way. I am very fortunate to count with his guidance, wisdom and friendship. 
ABSTRACT OF THE THESIS

PARASEXUALITY AND HETEROKARYOSIS IN FUSARIUM OXYSPORUM

EORMA SPECIALIS CUBENSE

by

Blanca R. Cortes

Florida International University, 1995

Miami, Florida

Professor David N. Kuhn, Major Professor

Intra and Inter Vegetative Compatibility Group (VCG) heterokaryon formation was observed in Fusarium oxysporum f.sp. Cubense (FOC). Using the double-pick method to force heterokaryon formation via hyphal anastomosis, $10^{4}$ pairwise combinations were done between 100 auxotrophic mutants of FOC representing races 1,2 and 4 in five VCGs. Inter formae speciales heterokaryon formation was also observed on pairings between benomyl resistant wild Type Testers (WTT) and wild type isolates in 15 VCGs and four different formae speciales. Microconidia analysis of heterokaryons identified both parental phenotypes as well as diploid phenotypes. Colonies with altered and hybrid genotypes were recovered from analysis of sectors from prototrophic hybrids as well as sectors with parental phenotype providing 
evidence of all stages of the parasexual cycle in this asexual, soilborne, phytopathogen. 


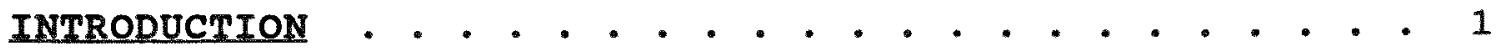

The Parasexual cycle. . . . . . . . . . . . . 4

Heterokaryon Formation . . . . . . . . . . 5

Karyogamy . . . . . . . . . . . . . . . 7

Mitotic Recombination ..... . . . . . . 7

Haploidization/Aneuploidy . . . . . . . . . 8

objectives

MATERIALS AND METHODS . . . . . . . . . . . . . . . . . . 11

strains . . . . . . . . . . . . . . . . . . 11

Culture Media . . . . . . . . . . . . . . . . 11

Protocols . . . . . . . . . . . . . . . . 11

THE EXPERIMENTAL DESIGN . . . . . . . . . . . . . . . . 13

Generation of Ausotrophic Mutants . . . . . . . . . 13

Heterokaryon Formation . . . . . . . . . . . . . . 16

Diploid Formation ................. . 18

Haploidization . . . . . . . . . . . . . . 19

EXPECTATIONS . . . . . . . . . . . . . . . 20

RESULTS . . . . . . . . . . . . . . . . . . . . 21

Generation of mutants in FOC . . . . . . . . . 21

Heterokaryon Formation in FOC . . . . . . . . . . 28

Hybrid Formation in FOC . . . . . . . . . . . . .78

Altered Parental Phenotypes . . . . . . . . . . 84

Haploidization... . . . . . . . . . . . . 86

CONCLUSION . . . . . . . . . . . . . . . . . 89

LIST OF REFERENCES . . . . . . . . . . . . . . . 93

APPENDIXES . . . . . . . . . . . . . . . . . 101

Appendix A: strains of FOC ........... 102

Appendix B: Media Preparation.......... 103

Appendix C: Protocols.............. 105

Appendix D: Auxotrophic Mutants of FOC . . . . 120

Appendix E: Pairings Between Auxotrophic Mutants 126 
TABLE 1 Mutagenized wild Type strains of Foc . . . . . . 22

TABLE 2 Intra-strain Heterokaryon Formation in VCG 120 . 31 TABLE 3 Intra-strain Heterokaryon Formation in VCG 126 - 31 TABLE 4 Inter-strain/Inter-race Het Formation in VCG 12031 TABLE 5 VCG 120 and VCG 126 Heterokaryon Formation. . 32 TABLE 6 Analysis of Microconidia from Transferred Plugs 36 Table 7 Diagnostic Test: Random Intra-VCG Pairings . . 37 Table 8 Diagnostic Test: Random Inter-VCG Pairings . . 38 Table 9 Intra VCG Heterokaryon Formation . . . . . . . 42 Table 10 Breakdown Intra VCG Heterokaryon Formation . . 43 Table 11 Inter-VCG Heterokaryon Formation . . . . . . . 44 Table 12 Intra-VCG vs. Inter-VCG Heterokaryon Formation - 45 Table 13 VCG 120: Intra-strain Isolates on MMA + Met . . 67 Table 14 VCG 124: Intra-strain Isolates on MMA + LYs . • 67 Table 15 VCG 126: Intra strain Isolates on MMA + LYs • . 67 Table 16 Hyphal Tip Analysis Intra-VCG Het Formation . 72 Table 17 Hyphal Tip Analysis Inter-VCG Het Formation . . 72 Table 18 Heterokaryon Formation using wild Type Testers . 75 Table 19 Intra VCG Hybrid Formation . . . . . . . . . . 80 Table 20 Inter VCG Hybrid Formation. . . . . . . . . . 81 Table 21 Hybrid Enhancement . . . . . . . . . . . . 82 Table 22 Diagnostic Test Enhanced Microconidia . . . . . 83 TABLE 23 Hyphal Tip Analysis of Hybrids....... . . . 83 Table 24 Analysis of sectors of Hybrids......... . 88 
Figure 1 Distribution auxotrophs VCG 0120 . . . . . 25

Figure 2 Distribution auxotrophs VCG 0124 . . . . . . . 26

Figure 3 Distribution auxotrophs VCG 0126 . . . . . . 27

Figure 4 Het Formation 22425 . . . . . . . . . . . . 46

Figure 5 Intra-VCG Incomptibility vs Inter-VCG Compatibility 22425 . . . . . . . . . . . 47

Figure 6 Het Formation SA8 . . . . . . . . . . . 48

Figure 7 Intra-vCG Incomptibility vs Inter-vCG Compatibility SAB .............. . 49

Figure 8 Het Formation STGM1 ............. 50

Figure 9 Intra-VCG Incomptibility vs Inter-vCG compatibility STGM1 . . . . . . . . . . 51

Figure 10 Het Formation STH1 . . . . . . . . . . . 52

Figure 11 Intra-VCG Incomptibility vs Inter-VCG Compatibility STH1 . . . . . . . . . . 53

Figure 12 Het Formation STC2 . . . . . . . . . . . 54

Figure 13 Intra-vCG Incomptibility vs Inter-vCG Compatibility STC2 ............. 55

Figure 14 Het Formation JCB1 . . . . . . . . . . . 56

Figure 15 Intra-VCG Incomptibility vs Inter-VCG Compatibility JCB1 ............. . 57

Figure 16 Het Formation GMB . . . . . . . . . . . . 58

Figure 17 Intra-VCG Incomptibility vs Inter-vCG Compatibility GMB ... . . . . . . . . . . 59

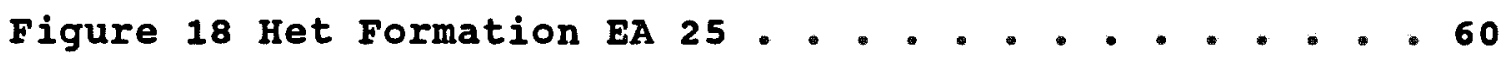

Figure 19 Intra-vCG Incomptibility vs Inter-VCG Compatibility EA 25 . . . . . . . . . . . 61

Figure 20 Het Formation STM3 .............. 62 
Figure 21 Intra-vCG Incomptibility vs Inter-VCG

compatibility STM3 ............. 63

Figure 22 Het Formation STB2 . . . . . . . . . . . . . . 64

Figure 23 Intra-VCG Incomptibility vs Inter-VCG compatibility STB2 ... . . . . . . . . 65

Figure 24 Analysis of 10,000 pairings in FOC . . . . . . 69

Figure 25 Distribution of auxotrophic mutants by vcG - 125 


\section{INTRODUCTION}

Fungi are important organisms for industry, medicine and agriculture. In the last decade, the advent of biotechnology has sparked a renewed interest in these organisms. However, most of the interest devoted to fungi stems from the variety of economically important plant pathogens. Many diverse and important plant pathogens are found in the genus Fusarium (Booth 1971). Species of these fungi, particularly Fusarium oxysporum, are responsible for vascular wilt of important agricultural crops such as tomato, carnation, melon, pea, corn and banana, to name but a few (Nelson 1981).

Species of Fusarium are divided into formae speciales based on the general host they infect and subdivided into pathogenic races based on the virulence of a pathogen to a specific host. Within a forma specialis, isolates are grouped into Vegetative Compatibility Groups (VCGs) (Ploetz 1990, Correll 1991). Members of the same VCG are genetically identical and are capable forming heterokaryons via hyphal anastomosis. However, vegetative incompatibility prevents heterokaryosis via hyphal anastomosis between members of different VCGs (Leslie 1993).

Incompatibility refers to the restriction that prevents two genotypes from coexisting in the same cytoplasm and it is a common occurrence between genetically different mycelia 
in higher fungi (Lane 1981). Incompatibility can prevent hyphal fusion and somatic nuclear exchange in filamentous fungi. Vegetative incompatibility is found in both sexual and asexual fungi, though, in general it does not affect sexual reproduction. Incompatible reactions are evidenced by barrage zones between neighboring vegetative hypha. Two forms of incompatibility are currently known, allelic and non-allelic. Allelic incompatibility occurs between strains that contain differences in the alleles at a single locus. Non-allelic incompatibility involves interactions between genes from a distinct locus.

Although vegetative incompatibility is regulated by incompatibility genes (vic genes, het genes) incompatibility reactions are expressed differently in different fungi. Podospora, shows non-allelic incompatibility where a single amino acid difference is sufficient to elicit an incompatible response (Deleu 1993) Neurospora on the other hand, shows allelic incompatibility and a single change in ideomorph of the het loci is responsible for vegetative incompatibility (Arganoza 1994, Glass and Lee 1992, Glass and Kuldau 1992).

Cell death has been reported to occur in non-self hyphal interactions (Aylmore 1986). Aspergillus will allow heterokaryon formation if a single allelic difference is present in the controlling allelic het gene but will elicit 
cell death if multiple differences are expressed (Anwar 1993, Dales 1990).

Partial-het genes have been identified in Aspergillus nidulans which confer partial heterokaryon incompatibility. That is, heterokaryon formation is observed but heterokaryotic growth is not as vigorous as heterokaryons formed between vegetatively compatible strains. Horizontal transfer, though, is not impaired by this partial incompatibility (Coenen 1994).

Despite vegetative incompatibility, heterokaryon formation is a common occurrence among fungal species (Arganoza 1994, Crawford 1986, Hocart 1979). In ascomycetes and basidiomycetes, heterokaryon formation occurs in the form of dikaryons. In ascomycetes, the dikaryotic stage is very unstable, with meiosis occurring almost immediately (Puhalla and Mayfield 1974). Basidiomycetes, on the other hand, can survive long periods of time as stable dikaryons before entering the sexual cycle (Raper 1966, Volk and Lenard 1989, Petersen and Ridley 1996).

Sexual cycles confer fungi great genetic diversity via segregation and allelic recombination. However, there are species of fungi with no known sexual cycle. Although asexual organisms are clonal, genetic diversity has been reported to occur in asexual fungi (Gordon 1992, Kafer 1960, Anderson 1995, Amoah 1995, Appel and Gordon 1995). One such 
case is documented for Fusarium oxysporum f.sp. cubense (FOC) (Leslie 1990, Ploetz 1990, Boehm 1994), an asexual, soilborne, phytopathogen responsible for the vascular wilt of banana (Stover 1990).

How do asexual organisms generate genetic diversity? How can new pathogenic races emerge if only the asexual state of the fungi is known?

Several mechanisms have been proposed to explain how asexual fungi generate genetic diversity, including spontaneous mutation, human intervention and parasexuality. Spontaneous mutation is a rare event and it would take a long period of time for the mutation to be established as a trait that can be recognized as characteristic of the strain. Human intervention refers to the actual transfer of an isolate from one infected site to another, either while harvesting or while marketing the crop. Parasexuality is defined as the horizontal transfer of genetic information without meiosis or the presence of sexual structures (Pontecorvo 1953).

\section{The Parasexual cycle}

There are four stages to the parasexual cycle: heterokaryon formation via hyphal anastomosis, karyogamy (fusion of the distinct nuclei), mitotic recombination and haploidization/segregation. 


\section{Heterokaryon Formation}

Heterokaryons are mycelia with more than one genetically distinct nucleus found in the same cell, which are not in the process of undergoing nuclear fusion. Heterokaryosis can be expressed in many forms (Burnett 1975). Transient heterokaryons are formed from the fusion of two different hyphae, a process known as hyphal anastomosis (Carling 1987, Cubeta 1993, Newhouse 1991). Although little is known about this process, it is believed that hyphal proximity elicits a response between hyphae (Ainsworth and Rayner 1986, 1989). If a repulsion reaction is triggered, cell death may occur and further fusion may be inhibited. However, if the hyphae recognize each other, contact is established, a pore opens between the hyphae and cytoplasmic and/or nuclear exchange may occur. The degree of acceptance between the nuclear and the cytoplasmic components of the forming heterokaryon determine its stability. The phenotype of a heterokaryotic cell is determined by the kind and the ratio of the different nuclei coexisting in the cell. Analysis of the progeny of a heterokaryon may be impaired when the hypha is multinucleate or coenocytic, thus, the advantage of using a septate, monokaryotic fungi such as Foc.

Several methods have been used to force heterokaryon formation between fungal isolates of Fusarium oxysporum, 
including protoplast fusion (Hocart 1994, Molnar 1990, Daboussi 1992, Gokhal 1992) and pairings using nitrate nonutilizing (nit) mutants (Correll 1987, Puhalla 1985, Ploetz 1990, Joaquim and Rowe 1990) and auxotrophic mutants (Buxton 1962, Dales 1983, Coenen 1994).

Protoplast fusion prevents vegetative incompatibility by removing the barriers of non-self recognition at the cell wall level, thus allowing heterokaryosis to occur. However, it is unlikely that isolates in nature would fuse by striping the markers from their cell walls.

The best evidence for compatibility comes from forcing heterokaryons between two complementary mutants. Nit mutants are normally used for this purpose (Cove 1976). Complementary nit mutants are allowed to grow toward each other on MMA plates. Nits grow sparsely on MMA but robust growth is observed on the area where the two isolates interact.

Auxotrophic mutants are also used to force heterokaryon formation. Auxotrophs are biochemical mutants that have lost the ability to synthesize necessary nutritional requirements.

Pairing color mutants can also be advantageous in determining if a heterokaryon was formed through analysis of microconidia from heterokaryotic colonies. 


\section{Kaxyogamy}

Karyogamy, the second stage of the parasexual cycle, is the fusion of two distinct nuclei and it is estimated to occur at a rate of one in $10^{6}$ spores from a heterokaryotic colony. Slow growing, prototrophic colonies emerging on MMA at low density from screened microconidia of heterokaryons are suggestive of diploid formation (Daboussi 1992, personal communication, our own results). It is during karyogamy that mitotic (somatic) recombination is thought to occur.

\section{Mitotic Recombination}

Until 1936 cross over was thought to be restricted to meiosis. The first ones to recognize mitotic recombination in fungi were Pontecorvo and Roper (1952) using Aspergillus nidulans as a model system. Mitotic crossover is thought to occur after chromosome replication and involves reciprocal exchange between two non sister chromatids (Roper and Pritchard 1955, Hastie 1968). The frequency of crossing over during mitosis is low (about 1 in 500 mitoses) (Burnett 1975). It is through somatic recombination that asexual fungi may generate genetic diversity. Unfortunately, the number of scorable recombinant phenotypes is not equivalent to the number of possible recombined genotypes and many recombination events go undetected. As fungi are normally haploid, segregation of chromosomes in diploid cells formed 
from the fusion of haploid nuclei is evidenced by fastgrowing sectors coming out of the diploid colony.

\section{Haploidization/Aneuploidy}

The final stage of the parasexual cycle is haploidization. In asexual organisms, haploidization is the non meiotic reduction of the diploid nuclei to its haploid components. It has been proposed that haploidization may occur through sequential chromosome loss by mitotic nondisjunction. Occasionally, an error in mitosis, during anaphase, causes two sister chromatids to move to the same pole, creating a non-disjunction event leading to aneuploidy (having chromosomes in a number different to the haploid set).

Aneuploids $(2 n+1$ or $2 n-1)$, are common products of first mitotic non-disjunction events and the condition is corrected by discarding the additional chromosomes. This segregation is evidenced by sectors coming out of the diploid colony.

Haploidization and/or aneuploidy can also be induced by several methods like UV mutagenesis (Wood 1969), benomyl (Anwar 1993) and p-fluorophenylalanine (Lhoas 1961, Gutz 1966). In some fungi, aneuploidy is more common than haploidization. In Foc, electrophoretic karyotype revealed a different number of chromosomes each time the isolates were 
tested, heavily suggesting an aneuploid state for isolates of FoC. (Boehm 1994).

\section{Current views}

Parasexuality is considered a rare event by the scientific community and many believe that the parasexual cycle, though present, might not be an important means of generating genetic diversity (Caten and Jinks 1966, Clutterbuck 1995).

However, the parasexual cycle has been identified in many fungi, (Pontecorvo 1953, Gil 1988, Durand 1992, Chacko 1994, Hocart 1994, L'OGarro 1992) including Fusarium species (Buxton 1956, 1962, Molnar 1990, Daboussi 1992). Moreover, evidence has been provided indicating that parasexual processes have been involved in recombination events (L'OGarro and Clarkson 1992, Molnar 1990, Daboussi 1992). Although Buxton (1962) reported recombination between single auxotrophs in Foc, many believed the evidence was not strong enough since the possibility of reversion could not be ruled out.

\section{objectives}

We propose that the parasexual cycle is a means by which asexual fungi may increase genetic variability. My goal, is therefore, defined as follows:

- To determine if all stages of the parasexual cycle are present in FOC. 
We chose Fusarium oxysporum f.sp. cubense (FOC) as a model to study parasexual processes for the following reasons: Pathogenic races of Foc infected banana cultivars during the 1950's forcing the introduction of new monoclonal banana cultivars resistant to this phytopathogen. However, within only 10 years of the introduction of this cultivar, Race 4, a new pathogenic race emerged, capable of infecting the new banana crops. Since no known sexual cycle has been identified for Foc, this genetic diversity is suggestive of a parasexual cycle in this phytopathogen.

In a practical aspect, Foc is a septate, uninucleate organism, with a global distribution. Fifteen Vegetative Compatibility Groups have been recognized worldwide. There are many strains within each VCG and three out of the four pathogenic races are represented. This variation allows for a wide range of interactions to be studied. In addition, Foc produces haploid, monokaryotic microconidia, which facilitates both, the analysis of progeny in heterokaryotic colonies and the screening of diploid formation.

Using 100 auxotrophic mutants of FOC (Appendix D) and the double-pick method (Appendix C) 10,000 pairwise combinations were made. Analysis of these pairings provided evidence of all stages of a parasexual cycle in Foc. 


\section{MATERIALS AND METHODS}

\section{strains}

The strains of Fusarium oxysporum f.sp. cubense used in this study are listed in Appendix $A$ and were obtained from the worldwide collection of Dr. Randy Ploetz, University of Florida, Institute of Food and Agricultural Sciences (Homestead, Florida). Formae speciales strains and species strains were provided by Dr. Corby Kistler, University of Florida, Institute of Food and Agricultural sciences (Gainsville, Florida). These strains are also listed in Appendix A.

\section{Culture Media}

The protocols used in the preparation of the media are described in Appendix B. From now on I will refer to the media used as follows:

I. Modified Czapeks Dox Complete Media (CMA)

II. Minimal media (MMA)

III. Potato Dextrose Broth (PDB)

IV. Benomyl - stock solution (CMB/MMB)

\section{Protocols}

The following protocols were used throughout this study. Each method is discussed in detail in Appendix $C$.

I. UV Mutagenesis (Finchman 1979).

II. Auxanography (Holliday 1956) 
III. Complementation Tests (Finchman 1979, Burnett 1975)

IV. Heterokaryon formation (Puhalla 1983, Cortes and Kuhn 1994)

V. Diagnostic Tests (Finchman 1979).

VI. Hybrid Enhancement (Cortes and Kuhn 1995)

VII. Hyphal tipping (Puhalla 1985) 


\section{THE EXPERIMENTAL DESIGN}

The first step in a parasexual analysis is to examine heterokaryon formation. Of the methods already described to force heterokaryon formation via hyphal fusion, we decided auxotrophic mutants would provide the most stringent screening approach to detect heterokaryons as neither "parent" can grow in unaugmented media yet prototrophic growth is observed if complementation has occurred. Since our ultimate goal was to screen diploid formation determine if parasexuality occurred in Foc if was important to achieve this stringency. It was, therefore, necessary to generate auxotrophic mutants in Foc to force heterokaryon formation.

\section{Generation of Auxotrophic Mutants}

Auxotrophic mutants can be generated with chemical mutagens or with radiation. We chose UV light as a mutagen to induce the mutations (Asthana and Tuveson 1992, Sasaki 1992, Graeme-Cook and Faull 1991, Rizwana and Powell 1995). UV mutagenesis damages DNA by forming dimers in adjacent thymine bases in the same DNA strand. Several repair mechanisms have evolved in eukaryotes to correct the damage: Photoreactivation, excision repair and the sos repair mechanism. The first two restore the DNA strand to its original shape and no mutations are usually detected. In 
sos repair, DNA synthesis by DNA polymerase bypasses the damaged area by an inducible replication system. The end result is a mutation in the DNA strand.

Occasionally, mutants go back to their prototrophic state, a process known as reversion.

To determine the intensity of UV radiation needed to yield a 50 survival rate on the mutagenized spores, kill curves were performed, using different doses of UV radiation on wild type strains. The high percent kill reduces the reversion rate of the induced mutations in the putative auxotrophs.

Strategies were designed to avoid losing putative auxotrophs to photoreactivation and to test for reversion. Mutagenesis (Appendix C) was done in the dark and the mutagenized spores were kept in the dark for a period of 24 hours to prevent light-activated enzymes from repair pathways to initiate repair of the damage DNA. After 24 hours the spores were filtered in the dark to remove mycelial growth of prototrophic colonies and/or germinated spores and returned to darkness for another 24 hours. Viability tests of the mutagenized spores were done on a daily basis for three days to determine how long it would take the spores to die. The test consisted of counting the number of spores with a hemacytometer and plating serial 
dilutions of the spore suspension on CMA plates. The number of colony forming units (cfus) was then compared against the counted number of spores to determine percent viability. In addition, an aliquot of the mutagenized spore suspension was plated daily on CMA plates for further diagnostic analysis. Using replica picking, the emerging cfus were screened for auxotrophs by picking on MMA and CMA plates (Appendix C). Putative auxotrophs grew on CMA plates but not on MMA plates. These colonies were tested and purified for three days through a series of single sporings. Auxanography of the nutritional deficiency of the mutants was determined with the Holliday Test (Holliday 1956) (Appendix C) .

To rule out reversion when screening prototrophic growth for putative heterokaryons or diploids, double auxotrophs were generated from single mutants. Reversion tests were conducted on the mutants by plating spores at high density (between $10^{6}$ and $10^{9}$ ) onto MMA plates. New kill curves were generated to determine if the number of mutations affected the sensitivity of these isolates to UV light and the UV dosage was adjusted accordingly. Only the isolates showing the lowest reversion rates were used for the pairings. 
Since we wanted to test the largest number of interactions among isolates of Foc, we chose to mutagenize VCGs with the largest number of strains and the highest race variability. VCG 0120 had more than 10 strains representing races 1 and 4 . VCG 0124 had more than five strains representing races 1 and 2 and VCG 0126 had four strains all from race 1.

To study heterokaryon formation in wild type populations, benomyl resistant auxotrophic mutants (Higgins 1987, Molnar 1990) (Appendix C) were generated to pair against available wild type strains of Foc and four different formae speciales.

\section{Heterokaryon Formation}

Once a pool of single and double auxotrophs and wild type testers were developed, the next step was to find a technique to pair these mutants. Several methods were used to force heterokaryon formation including coinoculation in Liquid Minimum Media, block pairings (Puhalla 1983) and a modified Buxton method (Appendix C). Although heterokaryon formation was observed in all three tested methods, the number of heterokaryons available for screening was limited. In order to increase the number of heterokaryons, a new method to force heterokaryon formation was developed, which I called the double-pick method (Appendix C). In the 
double-pick method a colony representing one parent is stabbed with a sterile toothpick at the edge and the inoculum is transferred to MMA plates. With a different sterile toothpick, the second parental colony is stabbed in the same fashion and co-inoculated in the same hole in which colony one was stabbed. Heterokaryon formation was seen within 72 hours and fifty to 100 individual pairwise combinations could be made in a single $9 \mathrm{~cm}$ petri dish. As a control, each parental strain was double-picked against themselves. Growth from the stabbed holes can be analyzed for heterokaryon formation using diagnostic tests (Appendix C).

Auxotrophs might be able to complement each other on minimal medium by cross feeding rather than by hyphal anastomosis (Correll 1986) so, to rule out cross feeding, double-picked pairings were conducted under conditions that would allow cross feeding but prevented hyphal anastomosis. Cellophane membranes were used for this test. Cellophane allows nutrients to diffuse but prevent physical contact between the hyphae, thus preventing anastomosis. Auxotrophic growth from microconidia separated by cellophane is an indication of cross-feeding. No growth indicates that hyphal anastomosis is required for complementation to occur. 
A strip of sterile cellophane was held vertically in a sterile petri dish by MMA. Each parental strain was picked on opposite sides of the cellophane strip. As a control, we double-picked the parents in the same hole without a cellophane barrier. To determine the amount of inoculum in each pick, the edge of the colony was stabbed and the toothpick was washed in $1 \mathrm{ml}$ sterile distilled water.

In haploid, uninucleate organisms like Foc, microconidia of heterokaryons should contain spores representing either parental phenotype that can be identified by screening on diagnostic plates (Appendix C) . Recovery of both parental phenotypes in screened colonies provides evidence of heterokaryon formation.

The ease of the double-pick method enabled me to force heterokaryon formation in large numbers of strain pairs.

\section{Diploid Formation}

Prototrophic growth of microconidia from heterokaryons on MMA plates at low density can be indicative of putative diploid formation. Spore analysis of putative diploids was made following the guidelines for screening the progeny of putative heterokaryons (Appendix C). The microconidia from putative diploids was further screened by hyphal tip analysis (Appendix C). 


\section{Haploidization}

Several techniques were used to induce haploidization in hybrid cells. Adenosine auxotrophs have a block in the biosynthetic pathways that induces the accumulation of a red pigment which confers on the colonies a red to pink color. These color auxotrophs were particularly useful in the screening of the haploidization stage, which could be detected as pink sectors coming out of white colonies. In addition, haploidizing agents were used to analyze putative hybrids including UV irradiation, p-fluorophenylalanine, benomyl treatments and the removal of selective pressure to induce breakdown. 


\section{EXPECTATIONS}

What did I expect to achieve with this experimental design?

- To generate a pool of mutants with many complementary auxotrophies in the selected VCGs.

- To force enough heterokaryotic events to screen patterns of heterokaryon formation and to be able to address these questions:

a) Is there any difference in the methods used to force heterokaryon formation? How stable are the heterokaryons formed?

b) Since mutants within the same strain would be generated from the same wild type isolate, do I see uniform patterns of intra-strain heterokaryon formation?

c) Since members within a VCG are genetically identical at the het loci, would I find an equal frequency of intra and inter-strain heterokaryon formation among them?

d) Would vegetative incompatibilities be observed in inter-VCG and inter-forma pairings?

- To screen enough progeny of heterokaryons to screen for hybrid formation

a) What's the frequency of hybrid formation? 
b) Are hybrids stable?

- To screen breakdown products of prototrophic colonies by forcing haploidization.

- To gather enough evidence to assess whether all stages of the parasexual cycle occur in FOC 
RESULTS

Generation of mutants in Foc

We mutagenized the following strains:

TABLE 1 Mutagenized Wild Type Strains of FoC

\begin{tabular}{||l|l|l||}
\hline VCG & STRAIN & RACE \\
\hline 120 & STGM1 & 1 \\
\hline & STH1 & 1 \\
\hline & STC2 & 1 \\
\hline & 22425 & 4 \\
\hline 124 & SA8 & 4 \\
\hline & STD1 & 1 \\
\hline & MACA & 1 \\
\hline & JCB1 & 2 \\
\hline & GMB & 1 \\
\hline & BLUG & 2 \\
\hline $124 / 5$ & STN1 & 1 \\
\hline 126 & EA & 1 \\
\hline & STB2 & 1 \\
\hline
\end{tabular}

Slight variations were observed in the sensitivity to UV light among the different strains. However, 5\% survival was achieved between $20 \mathrm{mj} / \mathrm{cm}^{2}$ and $25 \mathrm{mj} / \mathrm{cm}^{2}$. One hundred and sixty single, double and triple auxotrophic mutants were 
generated representing races 1,2 and 4 in all the tested VCGs. Comparing the kill curves of wild type strains, single auxotrophs and double auxotrophs we observed that double auxotrophs were more sensitive to UV light than single auxotrophs which were more sensitive to UV light than wild type strains.

Although we were expecting a wide array of induced mutations in these isolates, to our surprise we observed a high incidence of arginine, methionine and lysine auxotrophies. Of even greater interest was the fact that the frequencies which were obtained seemed to be strain related. For instance, 20 mutants out of 24 of the strain STGMI from VCG 0120 (Fig. 21) were deficient in arginine production, while the strain SA8 seemed to yield a greater number of methionine-deficient mutants. A higher incidence of methionine mutants was observed in strains from VCG 0124 (Fig. 22), while strains in VCG 0126 (Fig. 23) seemed to yield higher numbers of lysine mutants. One possible explanation for these differences could be that these strains are really aneuploid, therefore, able to mask mutations not observed.

Complementation tests were done on auxotrophies with the greater number of isolates (Appendix C). Damage to genes controlling different steps of the metabolic pathway 
is associated with enzymatic deficiencies which prevent the conversion of a substrate into an utilizable product for the next enzyme. However, when supplemented with the appropriate substrate, the pathway is restored until the final product is obtained.

Although we generated auxotrophs with damage on different steps of the tested biosynthetic pathways, once again we saw an increase in the incidence of isolates sharing the same damage point. For instance, STGM1 showed a high incidence of Arg2 mutants while SA8 showed a higher number of Met 4 mutants in the first mutation. However, the second mutation showed greater variability (Appendix D). 


\section{AUXANOGRAPHY ANALYSIS VCG 120}



Figure 1 


\section{AUXANOGRAPHY ANALYSIS \\ VCG 124}

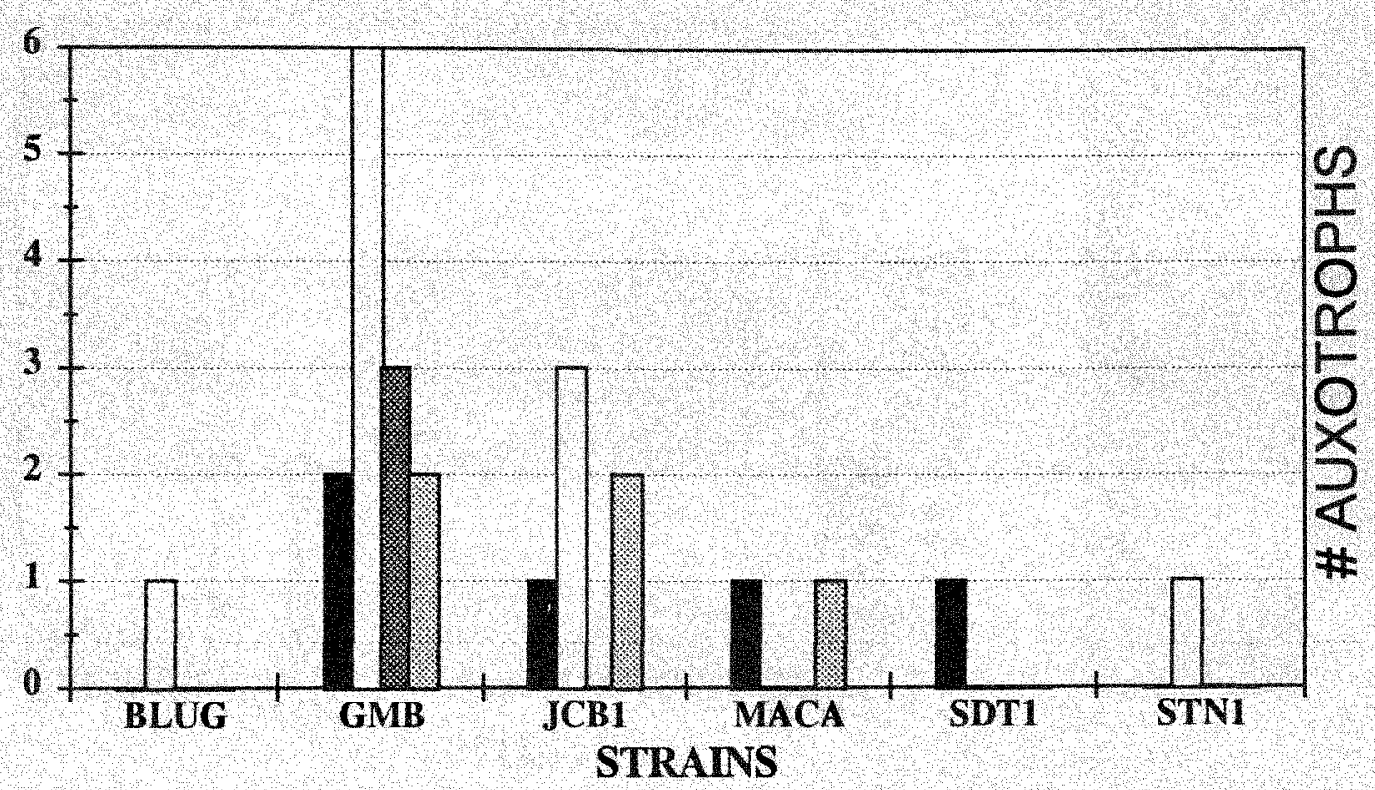

ARG

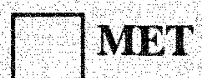

LYS

Figure 2 


\section{AUXANOGRAPHY ANALYSIS \\ VCG 126}

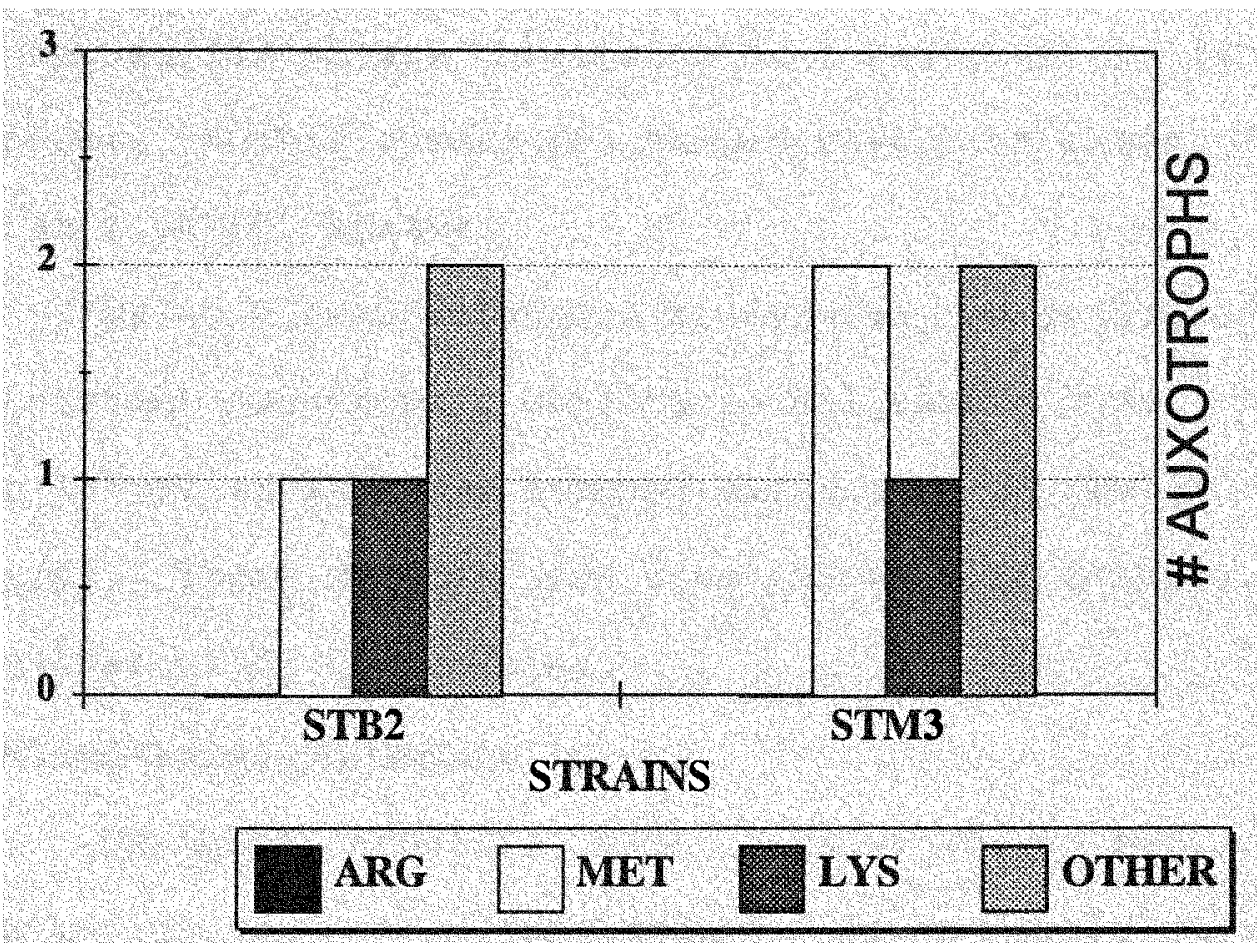

Figure 3 
Reversion tests were done on random strains with single and double auxotrophies. In general, reversion rates were observed for single auxotrophs at 1 revertant in $10^{6}$ to $10^{7}$ spores. Double auxotrophs showed reversion rates of fewer than 1 in $10^{9}$ spores.

Using direct selection procedures, we generated benomyl resistant auxotrophic mutants in all tested VCGs. About 1 in $10^{6}$ spores acquired benomyl resistance at levels of 100 $\mu \mathrm{g} / \mathrm{ml}$. These mutants were used in pairwise combinations against wild type strains.

Heterokaryon Formation in Foc

Using the generated auxotrophs we forced heterokaryon formation via hyphal anastomosis. Since double auxotrophs had the lowest reversion rates, they provided higher screening stringency. Using the methods described on the experimental design, we paired the auxotrophic mutants. Heterokaryon formation was observed in all tested methods. Cellophane tests (Appendix C) were performed and the possibility of cross feeding was discarded as no growth was observed in the areas separated by the cellophane membrane. Heterokaryon formation was observed in the control areas where both parents were allowed to interact without cellophane preventing anastomosis. 
Several important points came from the comparison of methods. First, there is no apparent difference in the frequency of heterokaryon formation due to the method used. Titrations done to force heterokaryons while using the Liquid MM method indicated that a minimum of $10^{5}$ spores was needed to generate heterokaryons. Daboussi (1992) in searching for hybrids, reported streaking spores of Nectria haematococca at a density lower than $10^{4}$ spores per plate to avoid heterokaryon formation. This information enabled me to screen for hybrid formation by plating the microconidia of heterokaryons on MMA plates at concentrations of $10^{3}$ to $10^{4}$ spores/plate.

The method that generated the higher number of available heterokaryons was the double pick method. Since the microconidia of heterokaryons are uninucleate, recovery of both parental phenotypes on screening the microconidia of the heterokaryons in all the tested methods provided evidence of two different nuclei present in the pool of spores (Tables 2-4). Both, intra-strain and inter-strain heterokaryon formation were observed. Pairwise combinations included intra-race/intra-strain and inter-race/inter-strain interactions.

Tables 2-4 represent analysis of microconidia of different pairwise combinations using the four methods 
described in The Experimental Design section. Recovery of both parental genotypes on the screened spores confirmed the heterokaryotic event. Of particular interest was the intrastrain pairing between STC2 Leu1 Lys2 and STC2 Pyr1

Adel(Table 2) in which colonies with altered parental phenotypes (putative recombinants) were recovered. 
TABLE 2 Intra-strain Heterokaryon Formation in VCG 120

\begin{tabular}{||l|l|l|l|l|l|l|}
\hline Method & Hets Formed & $\begin{array}{l}\text { Colonies on } \\
\text { MMA }\end{array}$ & $\begin{array}{l}\text { Colonies on } \\
\text { P1 only }\end{array}$ & $\begin{array}{l}\text { Colonies on } \\
\text { P2 only }\end{array}$ & $\begin{array}{l}\text { Colonies on } \\
\text { CMA }\end{array}$ & $\begin{array}{l}\text { Colonies on } \\
\text { P1 and P2 }\end{array}$ \\
\hline Double Pick & $87 / 100$ & $0 / 74$ & $6 / 74$ & $62 / 74$ & 74 & $6 / 74$ \\
\hline Block & $10 / 10$ & $0 / 73$ & $13 / 73$ & $47 / 73$ & 73 & $13 / 73$ \\
\hline Buxton & $3 / 3$ & $0 / 96$ & $7 / 96$ & $88 / 96$ & 96 & $1 / 96$ \\
\hline $\begin{array}{l}\text { Liquid MM } \\
\left(10^{6}\right)\end{array}$ & $1 / 1$ & $0 / 50$ & $11 / 50$ & $39 / 50$ & 50 & $0 / 50$ \\
\hline
\end{tabular}

P1 = STC2 Leu1 Lys2 P2= STC2 Pyr1 Ade1

TABLE 3 Intra-strain Heterokaryon Formation in VCG 126

\begin{tabular}{|l|l|l|l|l|l|}
\hline Method & Hets Formed & $\begin{array}{l}\text { Colonies on } \\
\text { MMA }\end{array}$ & $\begin{array}{l}\text { Colonies on } \\
\text { P1 only }\end{array}$ & $\begin{array}{l}\text { Colonies on } \\
\text { P2 only }\end{array}$ & Other \\
\hline Double Pick & $87 / 100$ & $0 / 49$ & $40 / 49$ & $9 / 49$ & $0 / 49$ \\
\hline Block & $8 / 10$ & $0 / 100$ & $55 / 100$ & $45 / 100$ & $0 / 100$ \\
\hline Buxton & $3 / 3$ & $0 / 49$ & $31 / 49$ & $18 / 49$ & $0 / 49$ \\
\hline $\begin{array}{l}\text { Liquid MM } \\
\left(10^{6}\right)\end{array}$ & $42 / 50$ & $0 / 98$ & $76 / 98$ & $22 / 98$ & $0 / 98$ \\
\hline
\end{tabular}

P1 $=$ STM3 Met1 Arg1 P2= STM3 Leu1 LYS2

TABLE 4 Inter-strain/Inter-race Het Formation in VCG 120

\begin{tabular}{|l|l|l|l|l|l|}
\hline \hline Method & Hets Formed & $\begin{array}{l}\text { Colonies on } \\
\text { MMA }\end{array}$ & $\begin{array}{l}\text { Colonies on } \\
\text { P1 only }\end{array}$ & $\begin{array}{l}\text { Colonies on } \\
\text { P2 only }\end{array}$ & Other \\
\hline Double Pick & $2 / 4$ & $0 / 270$ & $244 / 270$ & $26 / 270$ & $0 / 270$ \\
\hline Block & $4 / 5$ & $0 / 701$ & $658 / 701$ & $43 / 701$ & $0 / 701$ \\
\hline Buxton & $2 / 3$ & $0 / 416$ & $414 / 416$ & $2 / 416$ & $0 / 416$ \\
\hline $\begin{array}{l}\text { Liquid MM } \\
\left(10^{\wedge} 5\right)\end{array}$ & $4 / 5$ & $0 / 1108$ & $1096 / 1108$ & $12 / 1108$ & $0 / 1108$ \\
\hline $\begin{array}{l}\text { Liquid MM } \\
\left(10^{\wedge} 4\right)\end{array}$ & $2 / 2$ & $0 / 678$ & $666 / 678$ & $12 / 678$ & $0 / 678$ \\
\hline
\end{tabular}

P1 = SA8 Met4 Tyr1 P2= STGM1 Arg2 Ade1 
Of greater significance was the growth observed in inter-vegetative compatibility group interactions. If we follow the concept of Vegetative Compatibility Group, only those isolates within a VCG should be capable of heterokaryon formation via hyphal anastomosis and no interVCG heterokaryons should be detected. However, heterokaryon formation was detected on all four methods used (Table 5). Cross feeding was ruled out using the cellophane test (Correll 1986).

TABLE 5 Inter-VCG Het Formation between VCG 120 and VCG 126

\begin{tabular}{|l|l|l|l|l|l|l||}
\hline Method & $\begin{array}{l}\text { Hets } \\
\text { Formed }\end{array}$ & $\begin{array}{l}\text { Colonies } \\
\text { on MMA }\end{array}$ & $\begin{array}{l}\text { Colonies } \\
\text { on P1 only }\end{array}$ & $\begin{array}{l}\text { Colonies } \\
\text { on P2 only }\end{array}$ & $\begin{array}{l}\text { Colonies } \\
\text { on CMA }\end{array}$ & Other \\
\hline $\begin{array}{l}\text { Double } \\
\text { Pick }\end{array}$ & $50 / 50$ & $2 / 50$ & $15 / 50$ & $33 / 50$ & 50 & $0 / 50$ \\
\hline Block & $3 / 3$ & $0 / 100$ & $47 / 100$ & $53 / 100$ & 100 & $0 / 100$ \\
\hline Buxton & $3 / 3$ & $0 / 100$ & $49 / 100$ & $51 / 100$ & 100 & $0 / 100$ \\
\hline $\begin{array}{l}\text { Liquid } \\
\text { MM }\left(10^{6}\right)\end{array}$ & $15 / 15$ & 8 & 18 & 193 & 219 & 0 \\
\hline
\end{tabular}

P1 = STB2 Lys1 His1 Ade1 bent P2 = SA8 Met4 Pro1 bent

These unexpected results prompted us to postulate the following questions:

1. Is this inter-VCG heterokaryon an isolated event?

2. How stringent are the barriers imposed by the concept of vegetative incompatibility? 
3. How genetically distant may the isolates be to give rise to heterokaryon formation via hyphal fusion?

These questions required further analysis and we had the tools at hand to design an experiment that could provide possible explanations to our observations: a pool of auxotrophic mutants, and a method that allowed screening of a large number of heterokaryons at a time.

Using the double pick method 100 auxotrophic mutants of Foc were paired in all possible pairwise combinations and the pairings are represented in a grid (Appendix E). The auxotrophs used represented strains in VCG 0120, 0123, 0124, $0124 / 5$ and 0126 and races 1,2 and 4 . To facilitate analysis of the pairings on the grid, auxotrophic mutants were grouped by VCG. Within each VCG, mutants were grouped by auxotrophies, keeping together isolates within a strain sharing a nutritional deficiency. This arrangement allowed detection of complementation among similar as well as different deficiencies. Intra and inter strain combinations within a VCG could be screened. This arrangement also allowed at-a-glance detection of inter-VCG heterokaryon formation. Both, complementary and non-complementary interactions, were established.

Twenty-five different pairings were double picked in 9 cm MMA plates, for a total of 10,000 pairings. Both the 
isolates and the plates were identified with numbers to prevent biased scoring of the observed growth. Both parental strains were picked in the same plate as control. No growth was observed in the control area. Growth on the stabbed sites was scored in 48-72 hours after picking.

Sample plugs from random pairings representing intraand inter-race, intra- and inter-strain and intra- and inter-VCG were transferred to fresh MMA plates and LMM tubes and observed for continued growth. Serial dilutions of the spores from the LMM tubes were plated on MMA to assess continued growth (Table 6) and CMA as control. The first column in Table 6 itemize the pairings, a "+" in the second column indicates that the plug transferred to LMM continued to grow. Columns 3 and 4 represent serial dilutions of the microconidia of the putative heterokaryons. Platings at high density on MMA screens for heterokaryon formation while platings at low density on MMA screens for putative hybrid formation. The last column represents the control.

In general, the screened microconidia represented putative heterokaryon formation. However, putative hybrid formation was observed on some pairings such as STGM1 Arg2 X STGM1 Met4, STGM1 Arg2 X 22425 Leu2 Met1 Bent and STH1 Arg3 Ade2 Bent $X$ GMB Lys1 to name but a few. 
From the plugs transferred to MMA, spores were washed and screened on diagnostic plates (Appendix C) to determine the phenotype of the spores (Tables 7 and 8 ). Recovery of both parental phenotypes confirmed the heterokaryotic event. Prototrophic growth on MMA plated at $10^{3}$ spores was suggestive of putative hybrid formation. Putative recombinants included spores that could only grow on CMA, and spores that could grow on P1 and P2 plates but not on MMA. Further analysis of the colonies showing putative hybrid and recombinant phenotypes are described in the next chapters. 
TABLE 6 Analysis of Microconidia from Transferred Plugs

\begin{tabular}{|c|c|c|c|c|}
\hline PAIRING & LMM & $\operatorname{MMA}\left(10^{6}\right)$ & $\begin{array}{l}\text { MMA } \\
\left(10^{3}\right)\end{array}$ & CMA $\left(10^{3}\right)$ \\
\hline $22425 \operatorname{Arg} 3 \mathrm{X}$ GMB Lys & + & L & $\mathrm{L}$ & $\mathbf{L}$ \\
\hline 22425 Leu 2 X GMB Lys & + & $\mathrm{L}$ & 0 & 25 \\
\hline $\begin{array}{l}\text { SA8 URACIL X } \\
22425 \text { Leu2 Met1 B+ }\end{array}$ & + & $\mathrm{L}$ & 0 & 1 \\
\hline STGM1 Arg2 X STGM1 Met4 & + & $\mathrm{L}$ & 33 & 128 \\
\hline STC2 Arg1 X 22425 Arg 3 & + & 0 & 0 & 11 \\
\hline GMB Lys1 B $+X 22425$ Leu2 & + & $\mathrm{L}$ & 0 & 216 \\
\hline GMB Lys1 X 22425 Arg3 B + & + & $\mathrm{L}$ & $\mathbf{L}$ & $\mathbf{L}$ \\
\hline JCB1 Thi X SA8 Uracil & - & 0 & 0 & 0 \\
\hline $\begin{array}{l}\text { STGM1 Arg2 X } \\
22425 \text { Leu2 Met1 B + }\end{array}$ & + & L & 80 & 179 \\
\hline $\begin{array}{l}\text { STH1 Arg3 Ade2 B + X } \\
\text { GMB Lys1 }\end{array}$ & + & L & 43 & $\mathrm{C}$ \\
\hline $\begin{array}{l}\text { GMB Lys } 1 \mathrm{X} \\
\text { STC2 Pyr1 Ade1 }\end{array}$ & + & $\mathrm{L}$ & 0 & L \\
\hline $\begin{array}{l}\text { GMB Lys1 X } \\
\text { STGM1 Arg2 Ade1 }\end{array}$ & + & L & 0 & $\mathbf{L}$ \\
\hline STGM1 Arg2 X GMB Met4 & + & 67 & 4 & 17 \\
\hline STGM1 Arg2 X STC2 Pyr1 & + & $\mathrm{L}$ & 0 & $\mathbf{L}$ \\
\hline STH1 Arg $3 \times$ GMB Met4 & + & $\mathrm{L}$ & 30 & 128 \\
\hline $\begin{array}{l}\text { STH1 Arg3 Ade2 B + X } \\
22425 \text { Leu } 2 \text { Met1 B+ }\end{array}$ & + & $\mathrm{L}$ & 0 & $\mathbf{L}$ \\
\hline $\begin{array}{l}\text { STC2 Pyr1 Ade1 X } \\
22425 \text { Leu2 Met1 B + }\end{array}$ & + & L & 0 & $\mathbf{L}$ \\
\hline GMB Lys1 B + X STH1 Arg3 & + & $\mathrm{L}$ & 14 & 20 \\
\hline GMB Lys1 X STGM1 Arg2 & + & $\mathrm{L}$ & 0 & L \\
\hline STC2 Arg 3 X GMB Lys 1 & + & $\mathrm{L}$ & 0 & $L$ \\
\hline GMB Ade1 X STH1 Arg3 & + & 37 & 14 & 46 \\
\hline
\end{tabular}

L=Lawn; C=Contamination, unable to count; "+" = Positive growth; $"-"=$ no growth 
Table 7 Diagnostic Test: Random Intra-VCG Pairings

\begin{tabular}{|c|c|c|c|c|c|}
\hline PAIRINGS & P1 & $\mathbf{P 2}$ & MMA & CMA & A \\
\hline \multicolumn{6}{|c|}{ INTRA-STRAIN/INTRA-RACE/INTRA-VCG } \\
\hline $\begin{array}{l}\text { STGM1 Arg2 X } \\
\text { STGM1 Met1 } \\
\end{array}$ & 220 & 87 & 6 & 365 & 52 \\
\hline $\begin{array}{l}\text { STGM1 Met1 X } \\
\text { STGM1 Arg2 Adel }\end{array}$ & 54 & 24 & 0 & 78 & 0 \\
\hline $\begin{array}{l}\text { STM3 Met1 Arg3 X } \\
\text { STM3 Leu1 Lys2 }\end{array}$ & 202 & 94 & 0 & 296 & 0 \\
\hline $\begin{array}{l}\text { STC2 Leu1 Lys2 X } \\
\text { STC2 Pyr1 Ade1 } \\
\end{array}$ & 26 & 197 & 0 & 243 & 20 \\
\hline \multicolumn{6}{|c|}{ INTER-STRAIN/INTRA-RACE/INTRA-VCG } \\
\hline $\begin{array}{l}\text { SA8 Met4 X } \\
22425 \text { Arg3 } \\
\end{array}$ & 206 & 26 & 9 & 250 & 9 \\
\hline $\begin{array}{l}\text { STC2 Pyr1 Ade1 X } \\
\text { STH1 Arg3 Ade2 }\end{array}$ & 779 & 70 & 0 & 849 & 0 \\
\hline $\begin{array}{l}\text { STM3 Met1 Arg3 X } \\
\text { STB2 Lys1 Arg1 }\end{array}$ & 589 & 40 & 34 & 700 & 37 \\
\hline \multicolumn{6}{|c|}{ INTER-STRAIN/INTER-RACE/INTRA-VCG } \\
\hline $\begin{array}{l}\text { SA8 Met4 X } \\
\text { STGM1 Arg2 Ade1 }\end{array}$ & 8385 & 383 & 144 & 9027 & 115 \\
\hline $\begin{array}{l}22425 \text { Arg4 X } \\
\text { STH1 Arg3 Ade2 }\end{array}$ & 506 & 24 & 0 & 530 & 0 \\
\hline $\begin{array}{l}22425 \text { Arg3 X } \\
\text { STH1 Arg3 Ade2 }\end{array}$ & 2057 & 457 & 0 & 2514 & 0 \\
\hline $\begin{array}{l}22425 \text { Arg } 4 \text { X } \\
\text { STGM1 Met } 1 \\
\end{array}$ & 193 & 73 & 4 & 275 & 5 \\
\hline $\begin{array}{l}\text { SA8 Met4 Tyr1 X } \\
\text { STGM1 Arg2 Ade1 }\end{array}$ & 4696 & 321 & 0 & 5017 & 0 \\
\hline
\end{tabular}

$\mathrm{P} 1$ = Parent 1 genotype; $\mathrm{P} 2$ = Parent 2 genotype; $\mathrm{MMA}=$ Putative hybrid genotype; $\mathrm{CMA}=$ Control; $\mathrm{A}=\mathrm{Altered}$ Parental genotype (putative recombinant genotype) 
Table 8 Diagnostic Test: Random Inter-VCG Pairings

\begin{tabular}{|c|c|c|c|c|c|}
\hline PAIRINGS & $\mathbf{P 1}$ & $\mathbf{P} 2$ & MMA & CMA & $\mathbf{A}$ \\
\hline \multicolumn{6}{|c|}{ INTER-STRAIN/INTRA-RACE/INTER-VCG } \\
\hline $\begin{array}{l}\text { STB2 Lys1 Arg1B + X } \\
\text { STGM1 Met4 }\end{array}$ & 0 & 0 & 95 & 100 & 5 \\
\hline $\begin{array}{l}\text { STB2 Lys1 Arg1B + X } \\
\text { STH1 Arg } 3 \text { Ade2 B + }\end{array}$ & 237 & 10 & 53 & 300 & 0 \\
\hline $\begin{array}{l}\text { STC2 Pyr1 Ade1 X } \\
\text { MACA Leu2 }\end{array}$ & 86 & 14 & 0 & 100 & 0 \\
\hline $\begin{array}{l}\text { STC2 Pyr1 Ade1 X } \\
\text { STB2 Lys1 Arg1 B+ }\end{array}$ & 34 & 237 & 29 & 300 & 0 \\
\hline $\begin{array}{l}\text { STM3 Met1 Arg3 X } \\
\text { STC2 Leu1 Lys2 }\end{array}$ & 224 & 73 & 3 & 300 & 0 \\
\hline \multicolumn{6}{|c|}{ INTER-STRAIN/INTER-RACE/INTER VCG } \\
\hline $\begin{array}{l}\text { STB2 Lys1 HIS1 X } \\
\text { SA8 Met4 Arg3 }\end{array}$ & 6 & 290 & 4 & 300 & 0 \\
\hline
\end{tabular}

$\mathrm{P} 1$ = Parent 1 genotype; $\mathrm{P} 2$ = Parent 2 genotype; $\mathrm{MMA}=$ Putative hybrid genotype; $\mathrm{CMA}=$ Control; $\mathrm{A}=$ Altered Parental genotype (putative recombinant genotype). 
Several important interactions and patterns of heterokaryon formation were detected from these array of pairings. Tables 9-12 represent a summary of heterokaryon formation by strain. Table 9 looks at Intra-VCG interactions in general. Table 10 breaks down intra-VCG heterokaryons in intra-strain and inter-strain interactions, while Table 11 concentrates in inter-VCG heterokaryon formation. A comparison of intra-VCG heterokaryons vs inter-VCG heterokaryons is presented in Table 12.

Contrary to our expectations, there is great intrastrain variability when it comes to heterokaryon formation. Vegetative self-incompatibility was present in several of the tested strains, as intra-strain heterokaryon formation between complementary isolates was not detected. The frequency of heterokaryon formation differed from strain to strain. However, in general, isolates showing poor inbreeding affinity readily paired with complementary isolates in other strains. A higher incidence of inter-VCG pairings seemed to be observed in poor inbreeding strains (Figs. 4-23).

Figures $4,6,8,10,12,14,16,18,20$ and 22 represent heterokaryon formation broken down by isolate. Figures 5, 7, 9, 11, 13, 15, 17, 19, 21 and 23 represent the number of intra-VCG heterokaryons that should have been 
formed but did not compared to the number of inter-VCG heterokaryons that should not have occurred but did. The intra-strain variability can be appreciated in these figures.

For example, in VCG 0120 , all single mutants of strain SA8 came from the same wild type strain and most of the double mutants came from one single auxotroph (SA8 Met4). However, the frequency of heterokaryon formation varies from isolate to isolate (Fig. 6) SA8 is a strain that shows a low frequency of intra-strain heterokaryon formation but is very active in Inter-strain (Fig. 6) and Inter-VCG heterokaryon formation (Fig. 7).

Moreover, there are strains, like STH1 (Fig. 10) that seemed to favor intra-VCG heterokaryon formation over interVCG heterokaryon formation (Fig. 11). However, even when there are two auxotrophs in STH1, both sharing the same auxotrophies, different only in benomyl resistance, heterokaryon formation was different.

Similar patterns of heterokaryon formation were observed in VCG 0124 where GMB readily forms inter-VCG heterokaryons (Figs. 16 and 17) while JCB1, is very active in intra VCG heterokaryon formation and very poor in interVCG interactions(Figs. 14 and 15). 
The most active outcrosser was observed in the strain STB2 in VCG 0126. However, once again, variability was observed from isolate to isolate (Figs. 22 and 23) 
Table 9 Intra VCG Heterokaryon Eormation

\begin{tabular}{|l|l|r|r|r|}
\hline \hline VCG & STRAIN & $\begin{array}{l}\text { EXPECTED } \\
\text { OUTCOME }\end{array}$ & \multicolumn{1}{l|}{ OBSERVED } & $\%$ \\
\hline 0120 & 22425 & 242 & $150 / 242$ & 61.9 \\
\hline & STGM1 & 778 & $455 / 778$ & 58.5 \\
\hline & SA8 & 607 & $283 / 607$ & 46.6 \\
\hline & STH1 & 92 & $16 / 92$ & 17.4 \\
\hline & STC2 & 517 & $206 / 517$ & 39.8 \\
\hline 0123 & F9129 & 0 & 0 & 00.0 \\
\hline 0124 & MACA & 16 & $16 / 20$ & 80.0 \\
\hline & JCB1 & 96 & $83 / 96$ & 92.5 \\
\hline & GMB & 256 & $147 / 256$ & 57.4 \\
\hline & STD1 & 18 & $0 / 18$ & 00.0 \\
\hline $0124 / 5$ & EA23 & 2 & $0 / 2$ & 00.0 \\
\hline & EA25 & 4 & $3 / 4$ & 75.0 \\
\hline 0126 & STM3 & 40 & $42 / 40$ & 105.0 \\
\hline & STB2* & & $40 / 82$ & 48.8 \\
\hline \hline
\end{tabular}

"*" two "illegal" pairings were detected 
Table 10 Breakdown Intra VCG Heterokaryon Formation

\begin{tabular}{|l|l|r|r|r|r|r||}
\hline \hline VCG & STRAIN & OBSERVED & \multicolumn{1}{|l|}{$\begin{array}{l}\text { INTRA } \\
\text { STRAIN }\end{array}$} & \multicolumn{1}{l|}{$\begin{array}{l}\text { INTER } \\
\text { STRAIN }\end{array}$} & $\%$ \\
\hline 0120 & 22425 & $150 / 242$ & $4 / 16$ & 25.0 & $146 / 226$ & 64.6 \\
\hline & STGM1 & $455 / 778$ & $39 / 162$ & 24.1 & $416 / 616$ & 67.5 \\
\hline & SA8 & $283 / 607$ & $5 / 100$ & 05.0 & $278 / 507$ & 54.8 \\
\hline & STH1 & $16 / 92$ & $0 / 0$ & 0 & $16 / 92$ & 17.4 \\
\hline & STC2 & $206 / 517$ & $22 / 86$ & 25.6 & $184 / 431$ & 42.7 \\
\hline 0123 & F9129 & 0 & $0 / 0$ & 0 & $0 / 0$ & 0 \\
\hline 0124 & MACA & $16 / 20$ & $0 / 0$ & 0 & $16 / 20$ & 80.0 \\
\hline & JCB1 & $83 / 96$ & $17 / 20$ & 85 & $26 / 76$ & 34.2 \\
\hline & GMB & $147 / 256$ & $112 / 164$ & 68.3 & $35 / 92$ & 38.0 \\
\hline & STD1 & $0 / 18$ & $0 / 0$ & 0 & $0 / 18$ & 0 \\
\hline $124 / 5$ & EA23 & $0 / 2$ & $0 / 0$ & 0 & $0 / 2$ & 0 \\
\hline & EA25 & $3 / 4$ & $2 / 2$ & 100 & $1 / 2$ & 50.0 \\
\hline 0126 & STM3 & $40 / 82$ & $3 / 43$ & 6.9 & $37 / 39$ & 94.9 \\
\hline & STB2 & $42 / 40$ & $4 / 4$ & 100 & $38 / 40$ & 95.0 \\
\hline \hline
\end{tabular}


Table 11 Inter-VCG Heterokaryon Formation

\begin{tabular}{|l|l|r|r|}
\hline \hline VCG & STRAIN & \multicolumn{1}{l|}{$\begin{array}{l}\text { INTER- } \\
\text { VCG }\end{array}$} & \multicolumn{1}{c|}{$\%$} \\
\hline 0120 & 22425 & $30 / 181$ & 16.6 \\
\hline & STGM1 & $41 / 710$ & 5.7 \\
\hline & SA8 & $65 / 557$ & 11.7 \\
\hline & STH1 & $3 / 74$ & 4.1 \\
\hline 0123 & STC2B & $66 / 384$ & 17.2 \\
\hline 0124 & F9129 & $20 / 76$ & 26.3 \\
\hline & MACA & $13 / 71$ & 18.3 \\
\hline & JCB1 & $25 / 342$ & 7.3 \\
\hline & GMB & $89 / 879$ & 10.1 \\
\hline & STD1 & $3 / 52$ & 5.8 \\
\hline $124 / 5$ & EA23 & $6 / 91$ & 6.6 \\
\hline & EA25 & $27 / 171$ & 15.8 \\
\hline 0126 & STM3 & $42 / 522$ & 8.1 \\
\hline & STB2 & $115 / 565$ & 20.4 \\
\hline \hline
\end{tabular}


Table 12 Intra-VCG vs. Inter-VCG Heterokaryon Formation

\begin{tabular}{|l|l|r|r|r|r|}
\hline VCG & STRAIN & \multicolumn{1}{|l|}{ \% IAV } & \multicolumn{1}{|l|}{ \% IAS } & \% IES & \% IEV \\
\hline 0120 & 22425 & 61.9 & 25.0 & 64.6 & 16.6 \\
\hline & STGM1 & 58.5 & 24.1 & 67.5 & 5.7 \\
\hline & SA8 & 46.6 & 05.0 & 54.8 & 11.7 \\
\hline & STH1 & 17.4 & 0 & 17.4 & 4.1 \\
\hline & STC2 & 39.8 & 25.6 & 42.7 & 17.2 \\
\hline 0123 & F9129 & 00.0 & 0 & 0 & 26.3 \\
\hline 0124 & MACA & 80.0 & 0 & 80.0 & 18.3 \\
\hline & JCB1 & 92.5 & 85 & 34.2 & 7.3 \\
\hline & GMB & 57.4 & 68.3 & 38.0 & 10.1 \\
\hline & STD1 & 00.0 & 0 & 0 & 5.8 \\
\hline & EA23 & 00.0 & 0 & 0 & 6.6 \\
\hline $124 / 5$ & EA25 & 75.0 & 100 & 50.0 & 15.8 \\
\hline & STM3 & 48.8 & 6.9 & 94.9 & 8.1 \\
\hline 0126 & STB2 & 105.0 & 100 & 95.0 & 20.4 \\
\hline
\end{tabular}

Legend; iav=Intra VCG; ias=intra-strain; ies=inter-strain; iev=Inter VCG 


\section{HET FORMATION-22425}

V0120
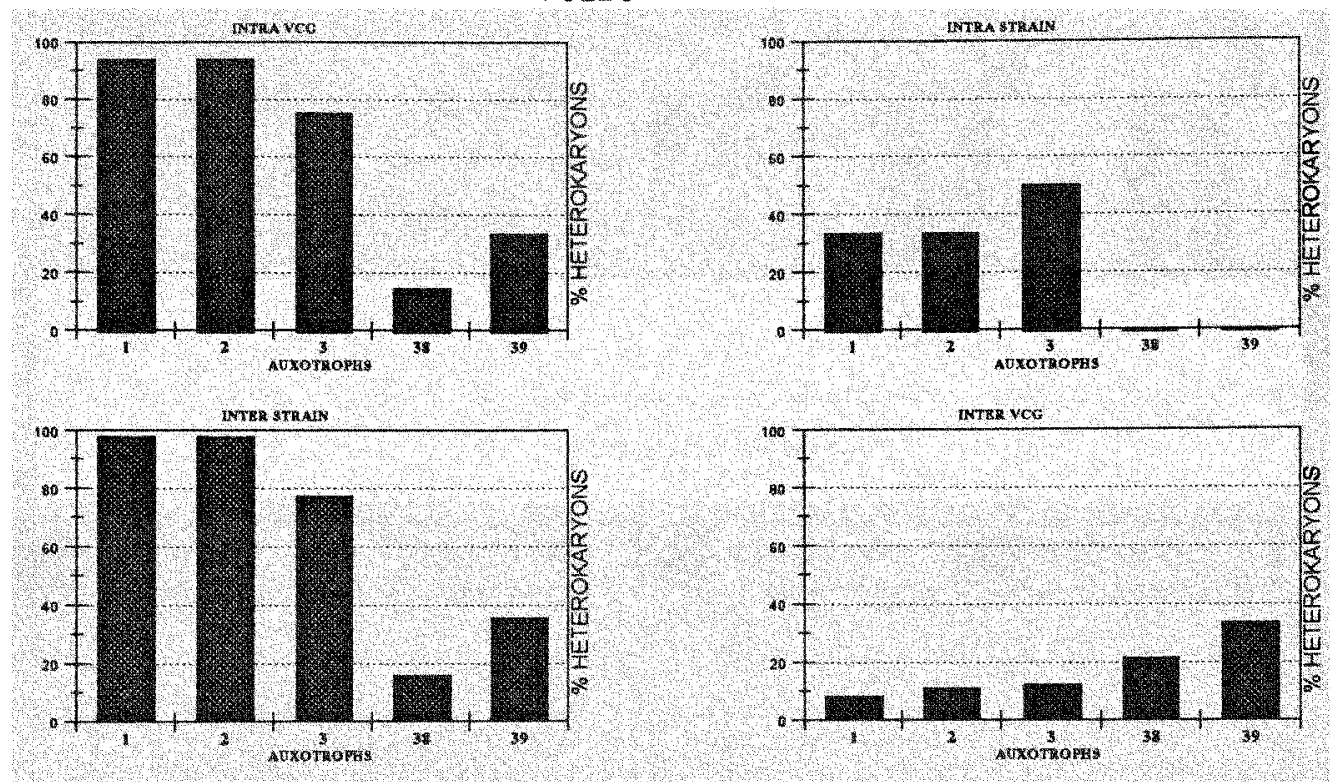

Figure 4 Patterns of heterokaryon formation in strain 22425. The isolates are as follows: \#1 Arg3 B+, \#2 Arg3, \#3 Arg4, \#38 Leu2Met1 and \#39 Leu2Met1 B+ 


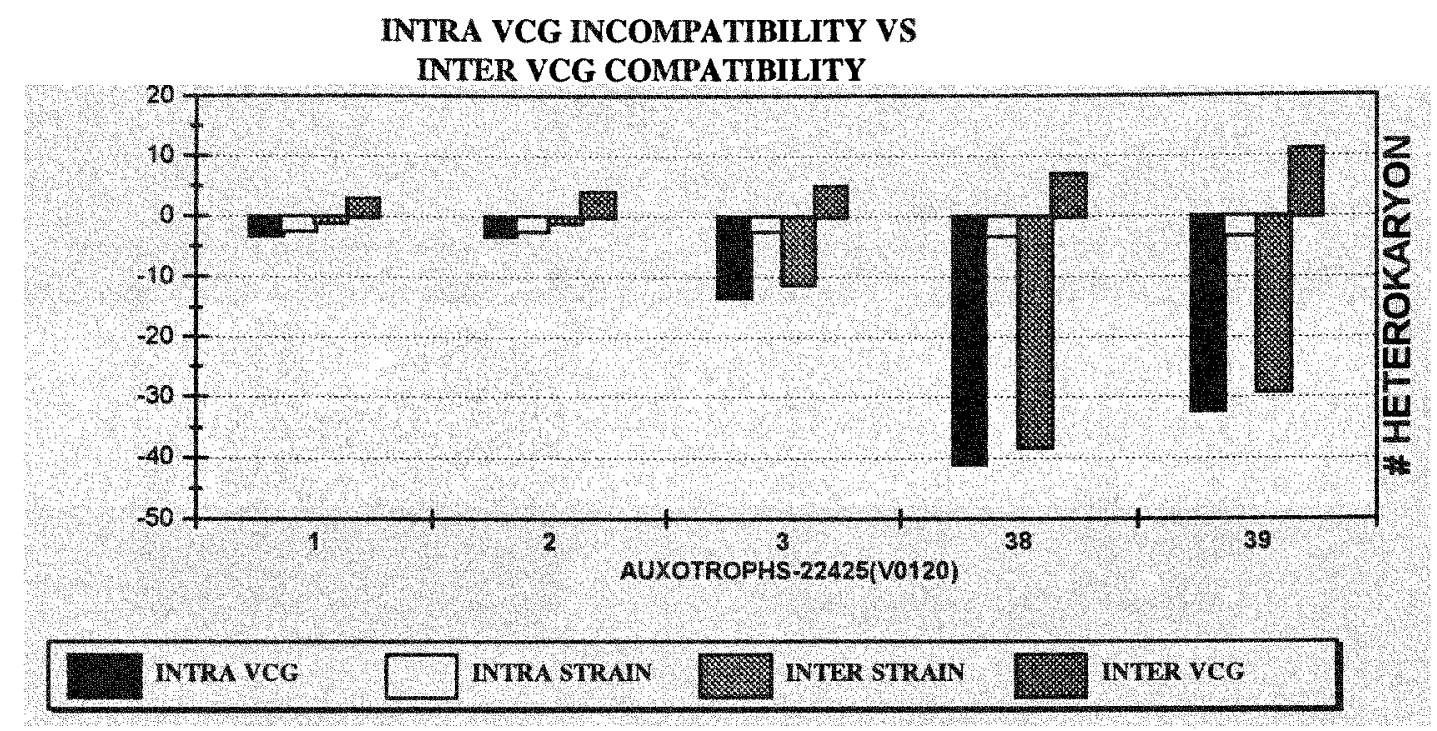

Figure 5 


\section{HET FORMATION-SA8}

V0120

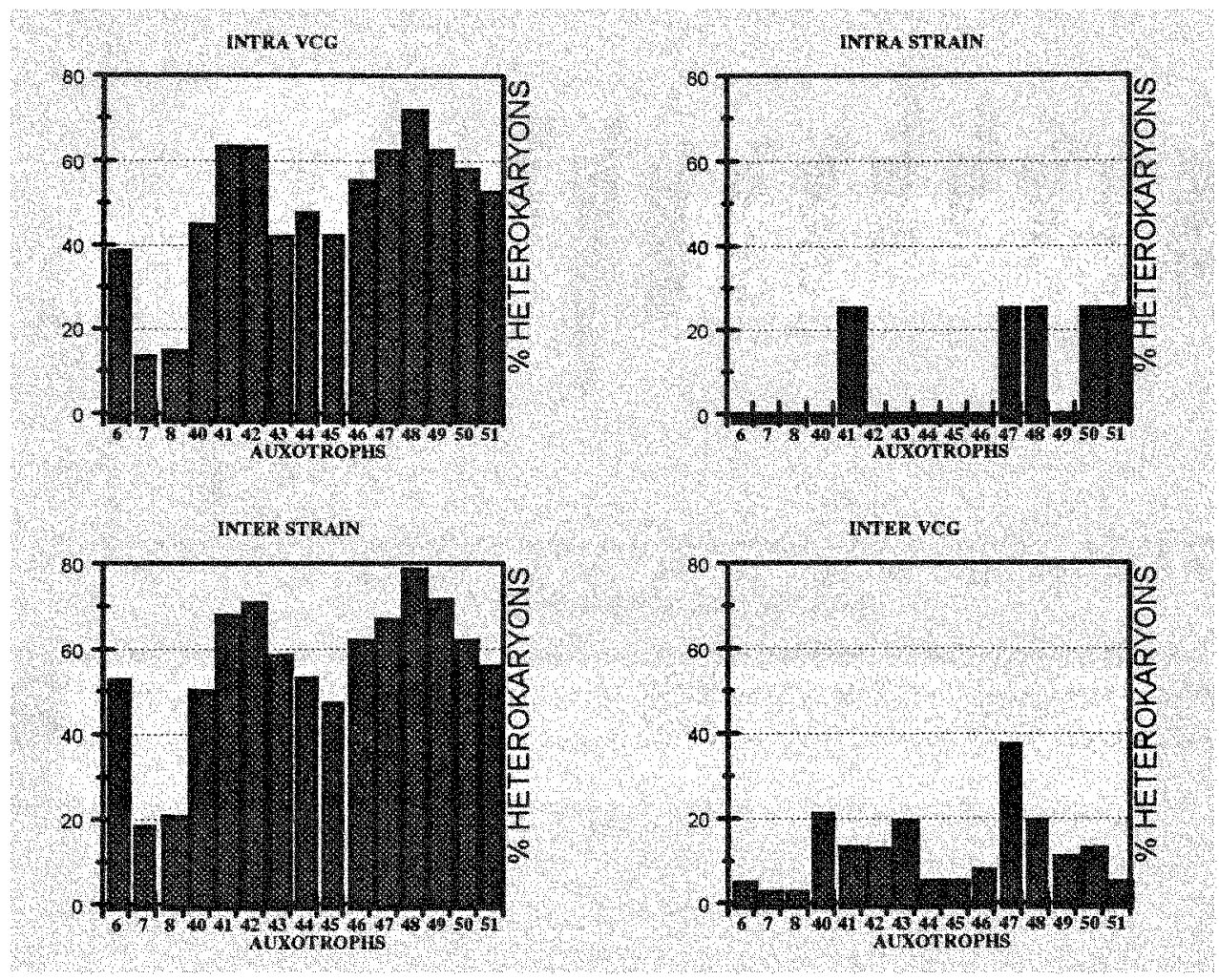

Figure 6 Patterns of heterokaryon formation in strain SA8. The isolates are: \#6 Uracil, \#7 His1, \#8 Ade1, \#40 Met4Tyr1 B+, \#41 Met4Tyr1, \#42 Met4Gly, \#43 Met2Lys1, \#44 Met4Pro1 B+, \#45 Met4Pro1, \#46 Met4Pro1, \#47Met4Arg1, \#48 Met4Arg3, \#49 Met4Arg3 B+, \#50 Met4 and \#51 Met4 


\section{INTRA VCG INCOMPATIBILITY VS INTER VCG COMPATIBILITY}

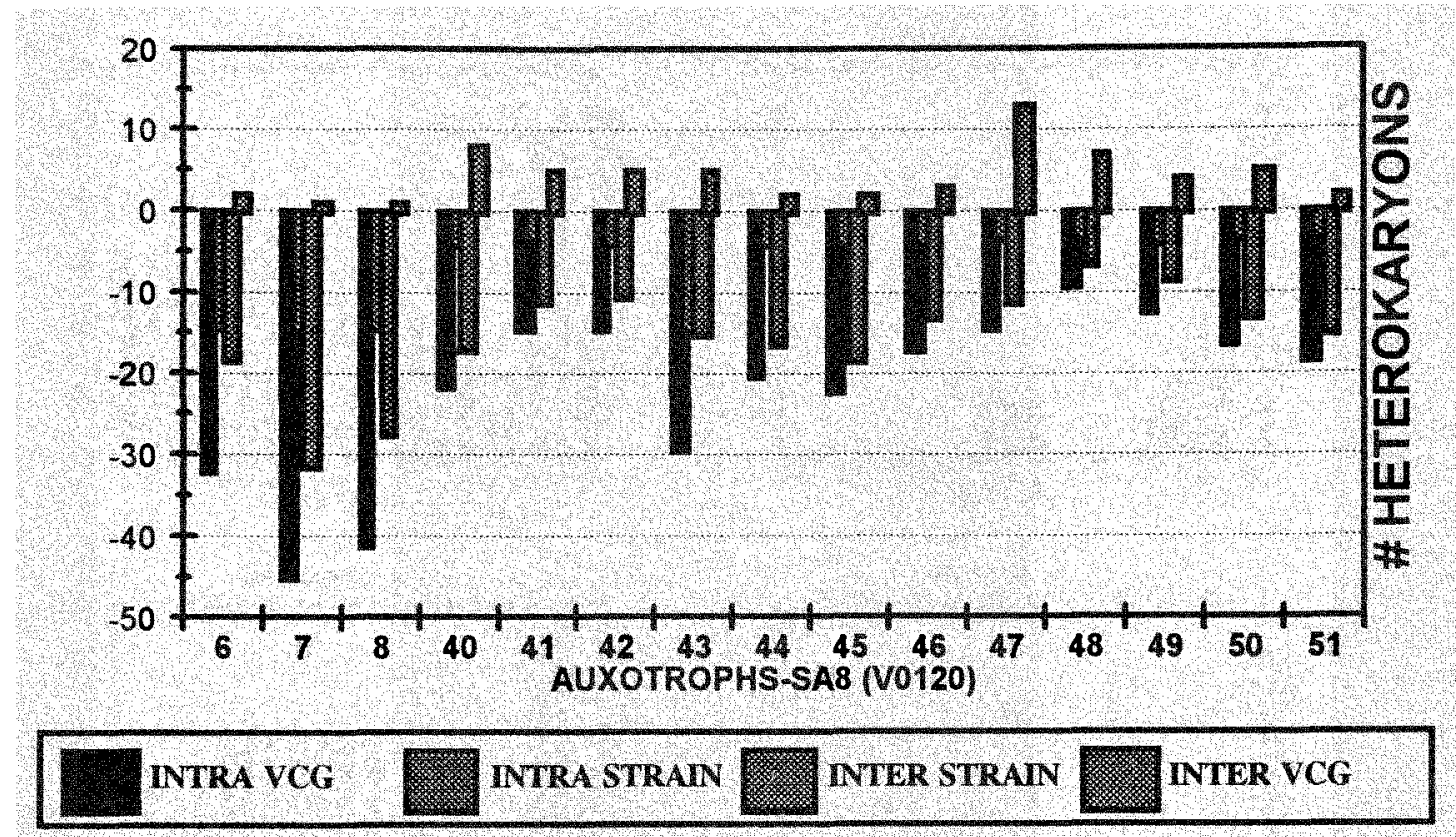

Figure 7 


\section{HET FORMATION-STGM1}

V0120
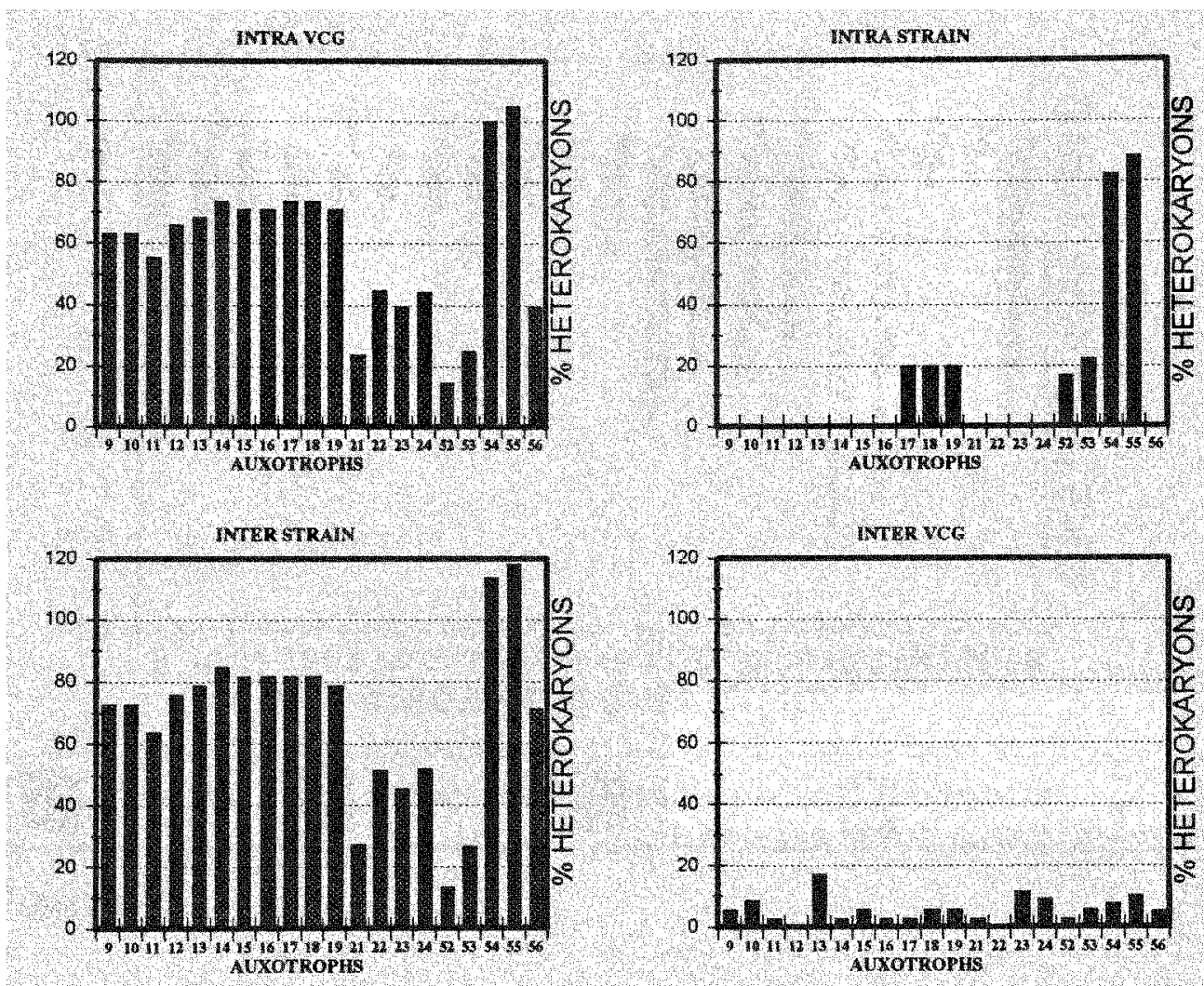

Figure 8 Patters of heterokaryon formation in strain STGM1. The isolates are: \#9 through \#23 Arg2, \#24 Arg2Ade1, \#52 Met1, \#53 Met1, \#54 Met4, \#55Met4 and \#56 Met4 


\section{INTRA VCG INCOMPATIBILITY VS INTER VCG COMPATIBILITY}

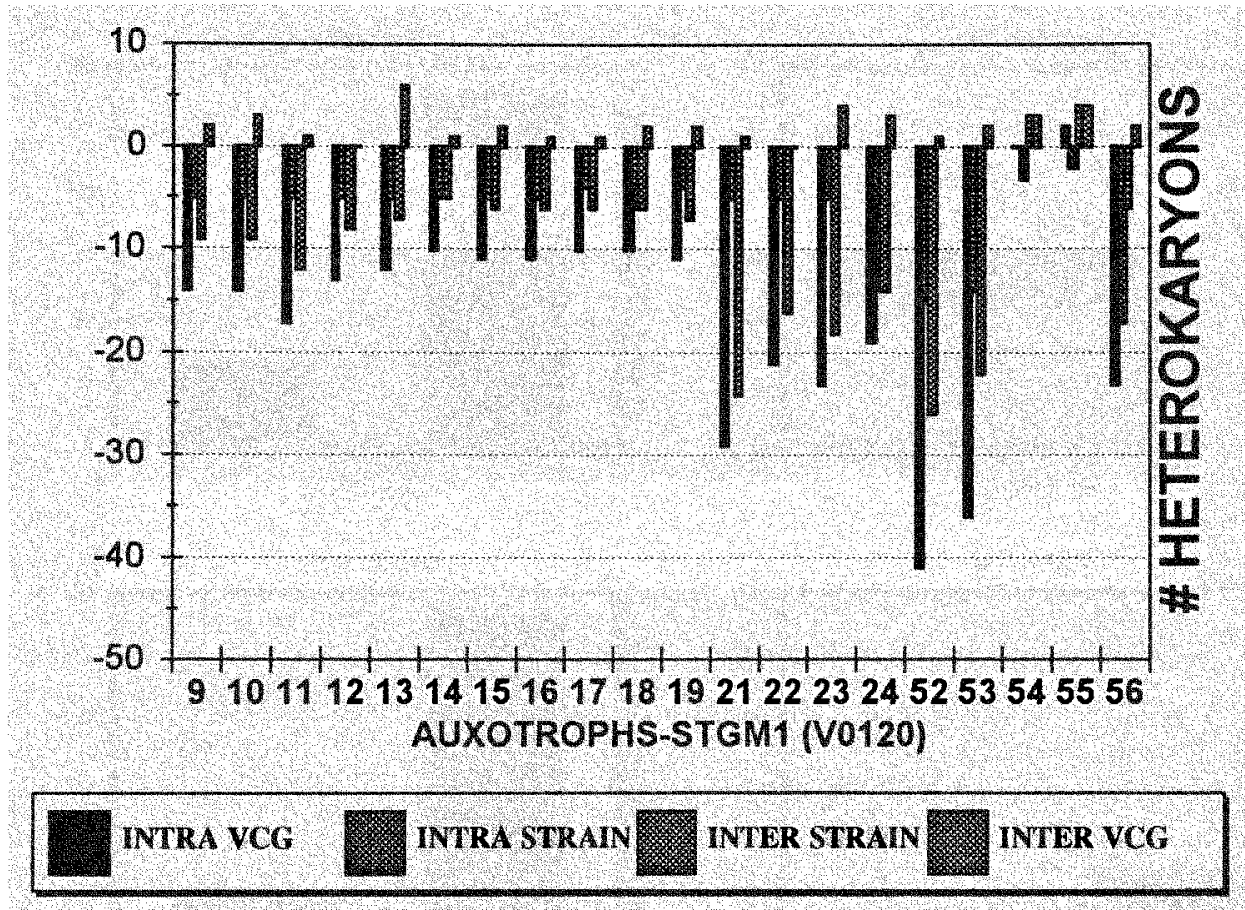

Figure 9 


\section{HET FORMATION-STH1 \\ V0120}
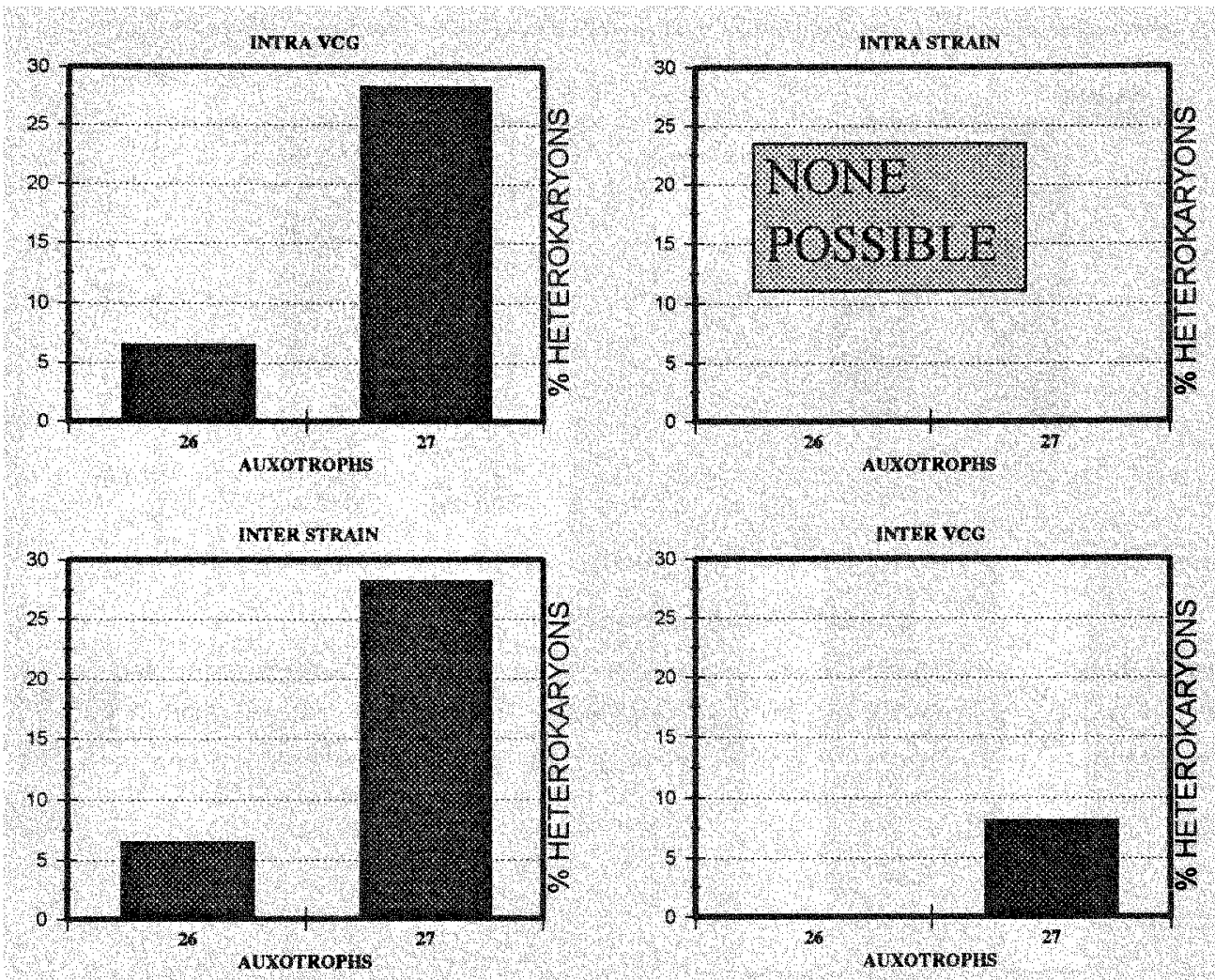

Figure 10 Patterns of heterokaryon formation in strain STH1. The isolates are: \#26 Arg3Ade2 and \#27 Arg3Ade2 B十 
INTRA VCG INCOMPATIBILITY VS

INTER VCG COMPATIBILITY

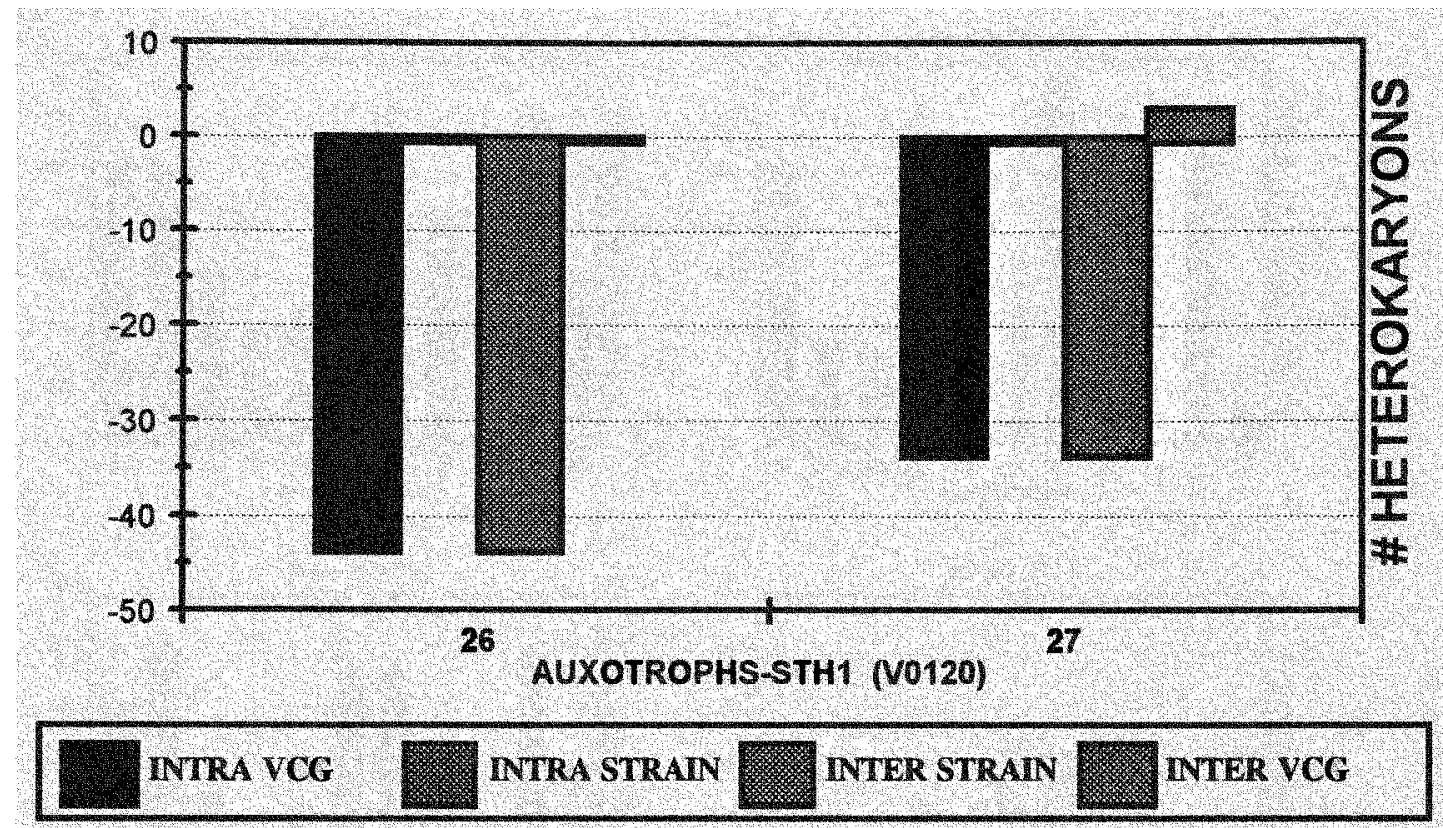

Figure 11 


\section{HET FORMATION-STC2}

\section{V0120}

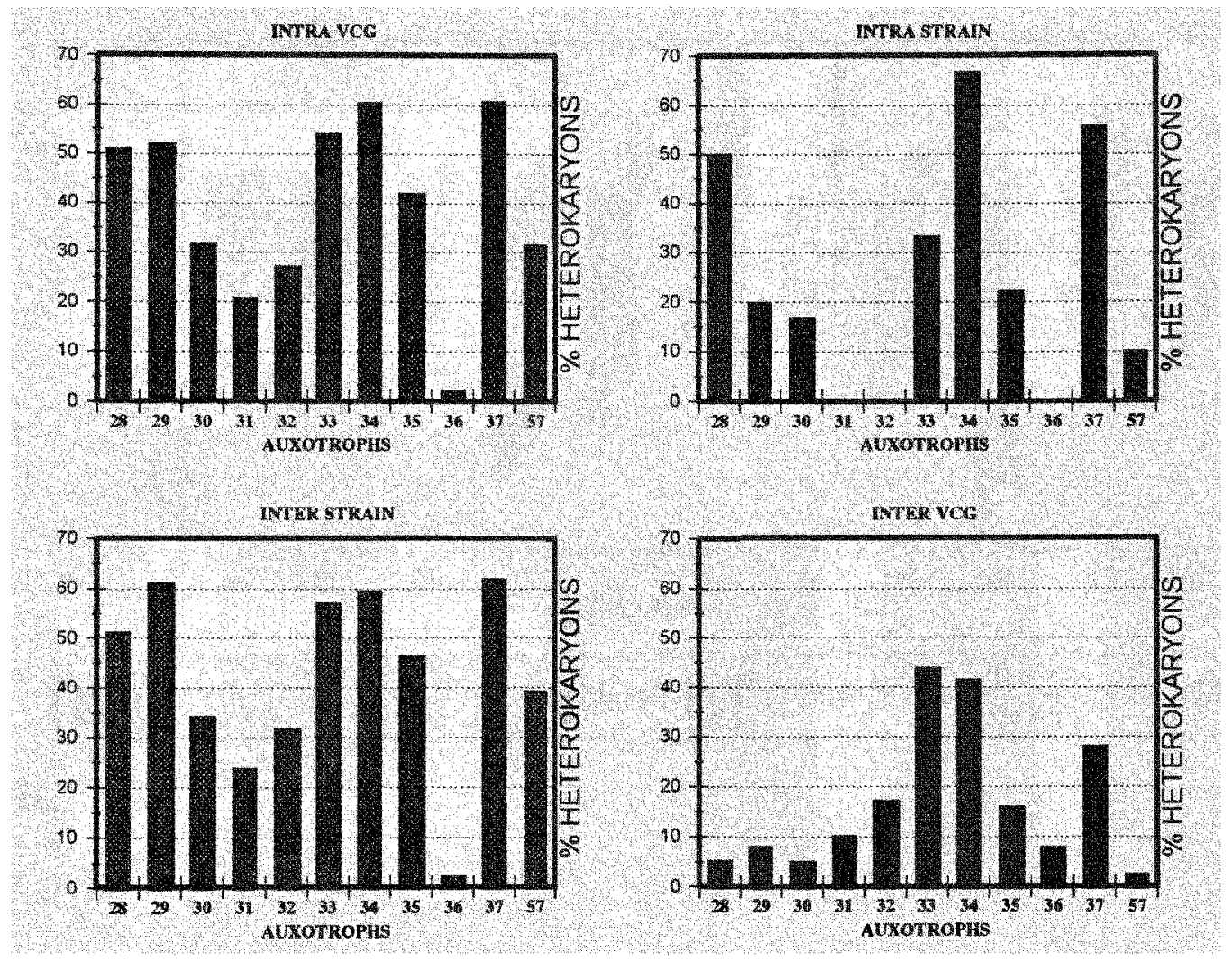

Figure 12 Patterns of heterokaryon formation in strain STC2. The isolates are: \#28 Arg1, \#29 Arg3, \#30 Pyr1Ade1, \#31 Pyr1Lys2, \#32 Pyr1Met1, \#33 Pyr1, \#34 Pyr1, \#35 Lys1, \#36 Leu1Lys1, \#37 Leu1, \#57 Met4 


\section{INTRA VCG INCOMPATIBILITY VS \\ INTER VCG COMPATIBILITY}

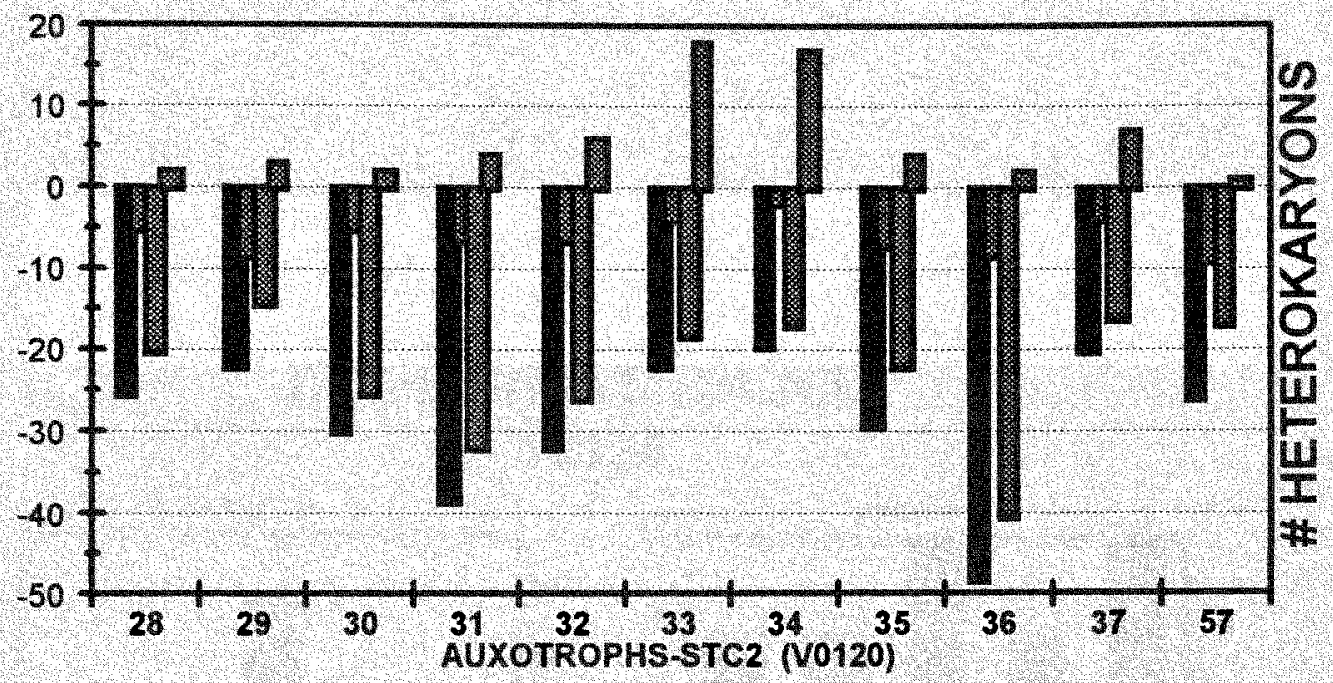

Figure 13 


\section{HET FORMATION-JCB1 \\ V0124}
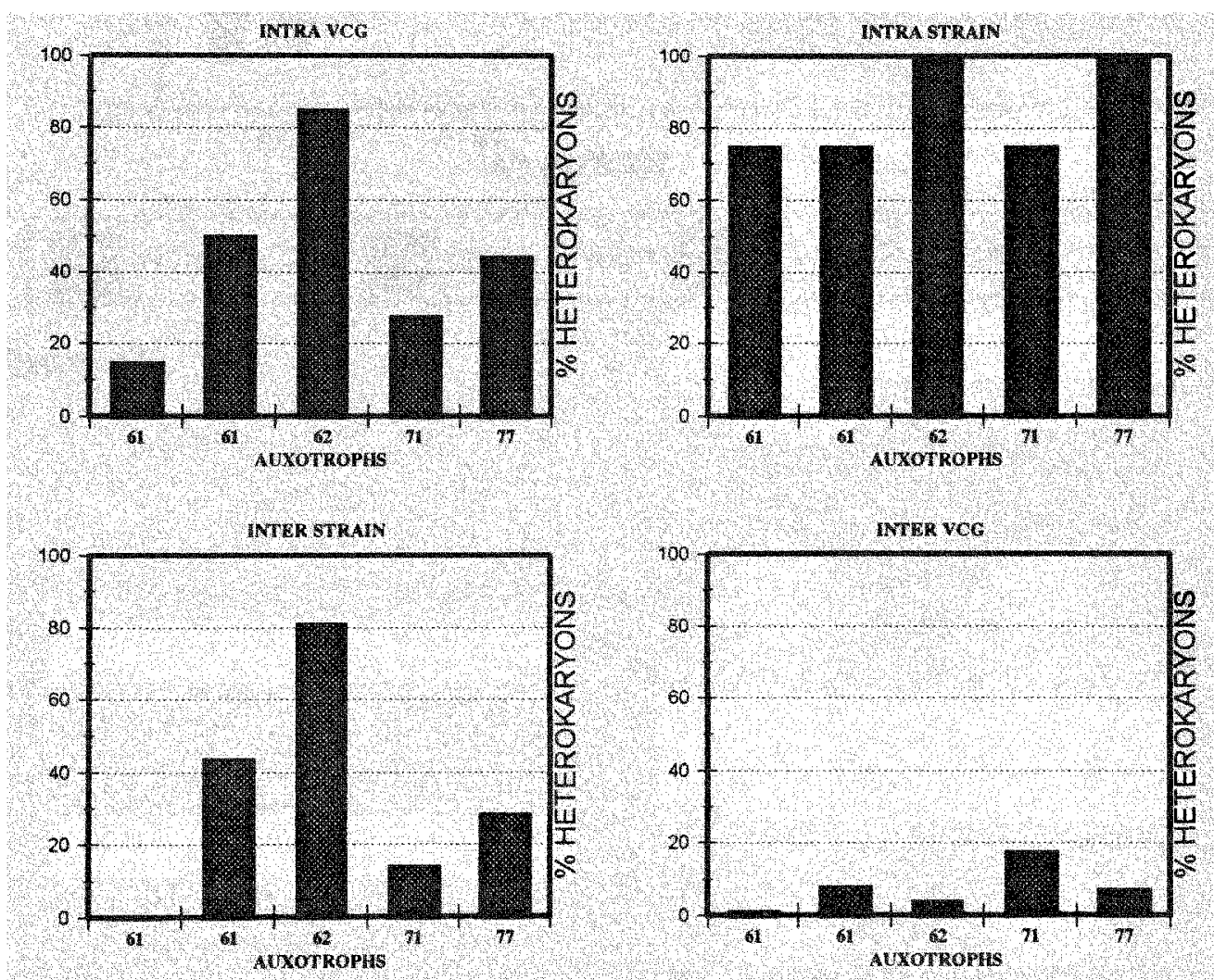

Figure 14 Patterns of heterokaryon formation in strain JCB1. The isolates are: \#61 Cys, \#61 Thi, \#62 unknown, \#71 Arg2 and \#77 Met1 


\section{INTRA VCG INCOMPATIBILITY VS \\ INTER VCG COMPATIBILITY}

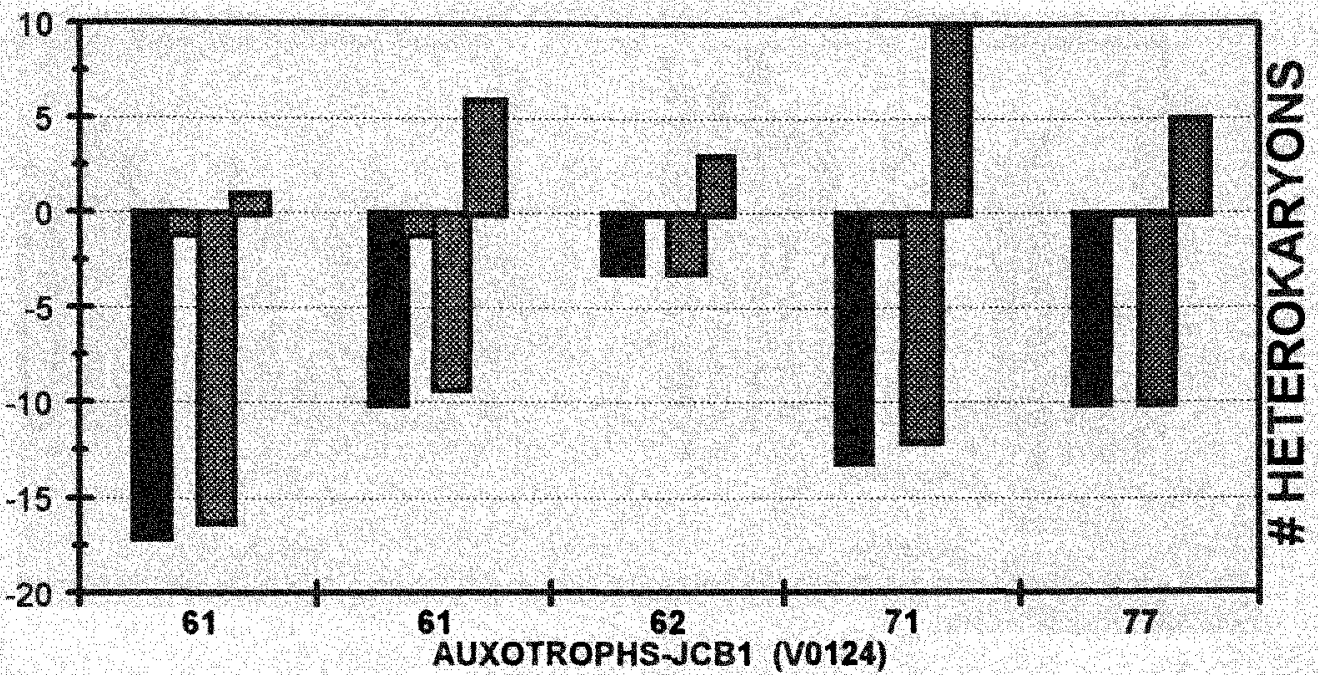

Figure 15 


\section{HET FORMATION-GMB}

\section{V0124}
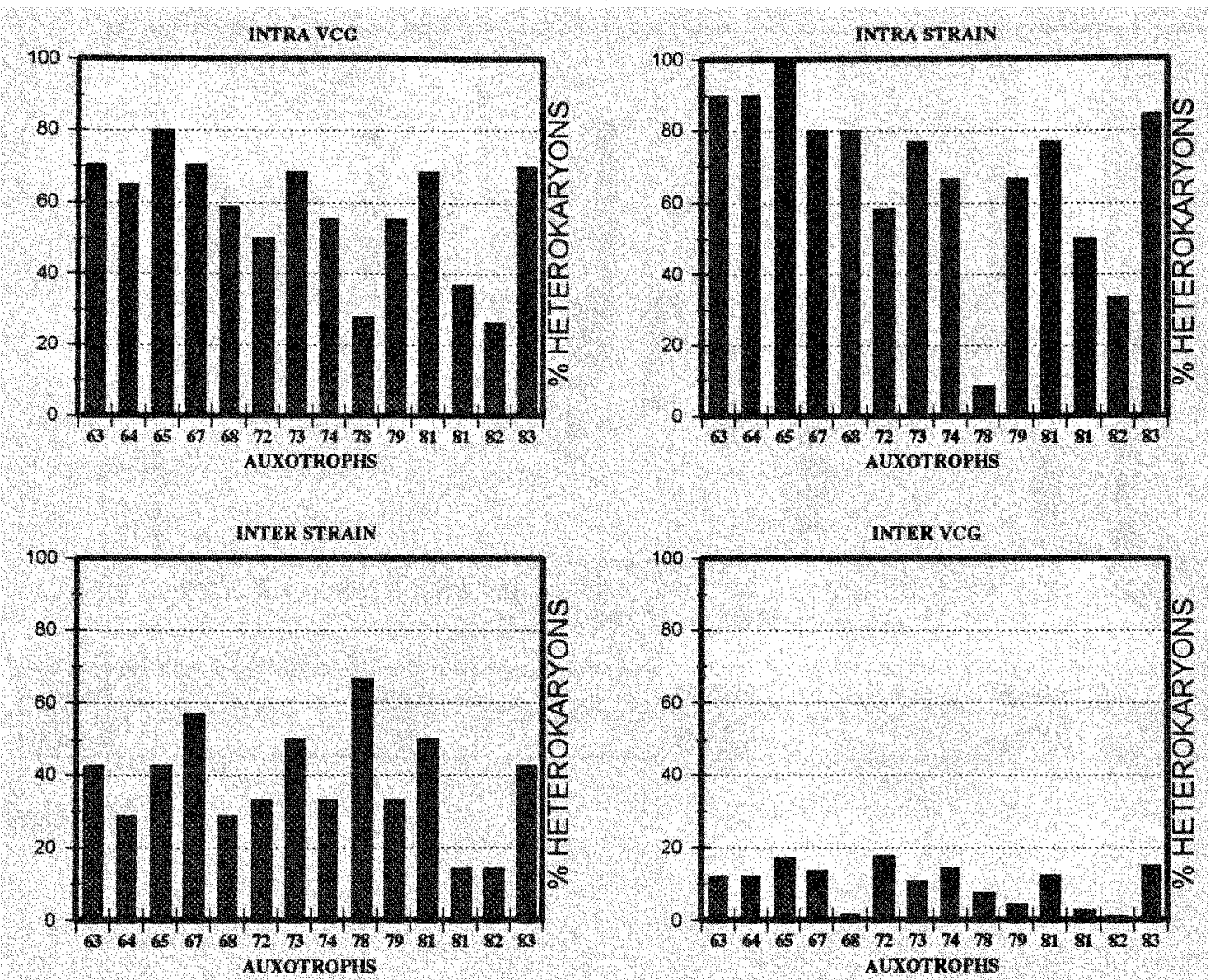

Figure 16 Patterns of heterokaryon formation in strain GMB. The isolates are: \#63 Lys1 B+, \#64 Lys1, \#65 Lys2, \#67 Lys1, \#68 Lys1Arg1, \#72 Arg2, \#73 Arg3, \#74 Arg2, \#78 Met1, \#79 Met1, \#81 Met 4, \#81 Ade1, \#82 Ade1 and \#83 unknown 


\section{INTRA VCG INCOMPATIBILITY VS \\ INTER VCG COMPATIBILITY}

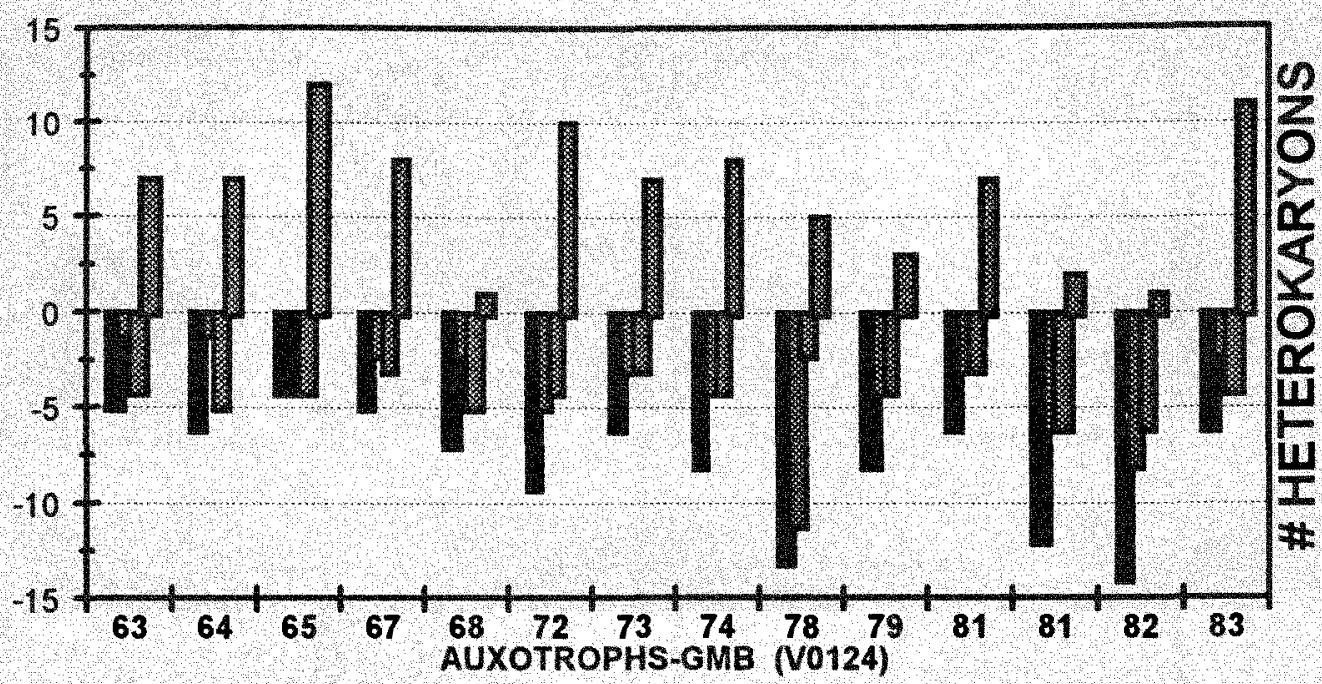

Figure 17 


\section{HET FORMATION-EA25}

\section{$\mathrm{V} 0124 / 5$}


Figure 18 Patterns of heterokaryon formation in strain EA 25. The isolates are: \#59 Pro1 and \# 75 Arg3 


\section{INTRA VCG INCOMPATIBILITY VS INTER VCG COMPATIBILITY}

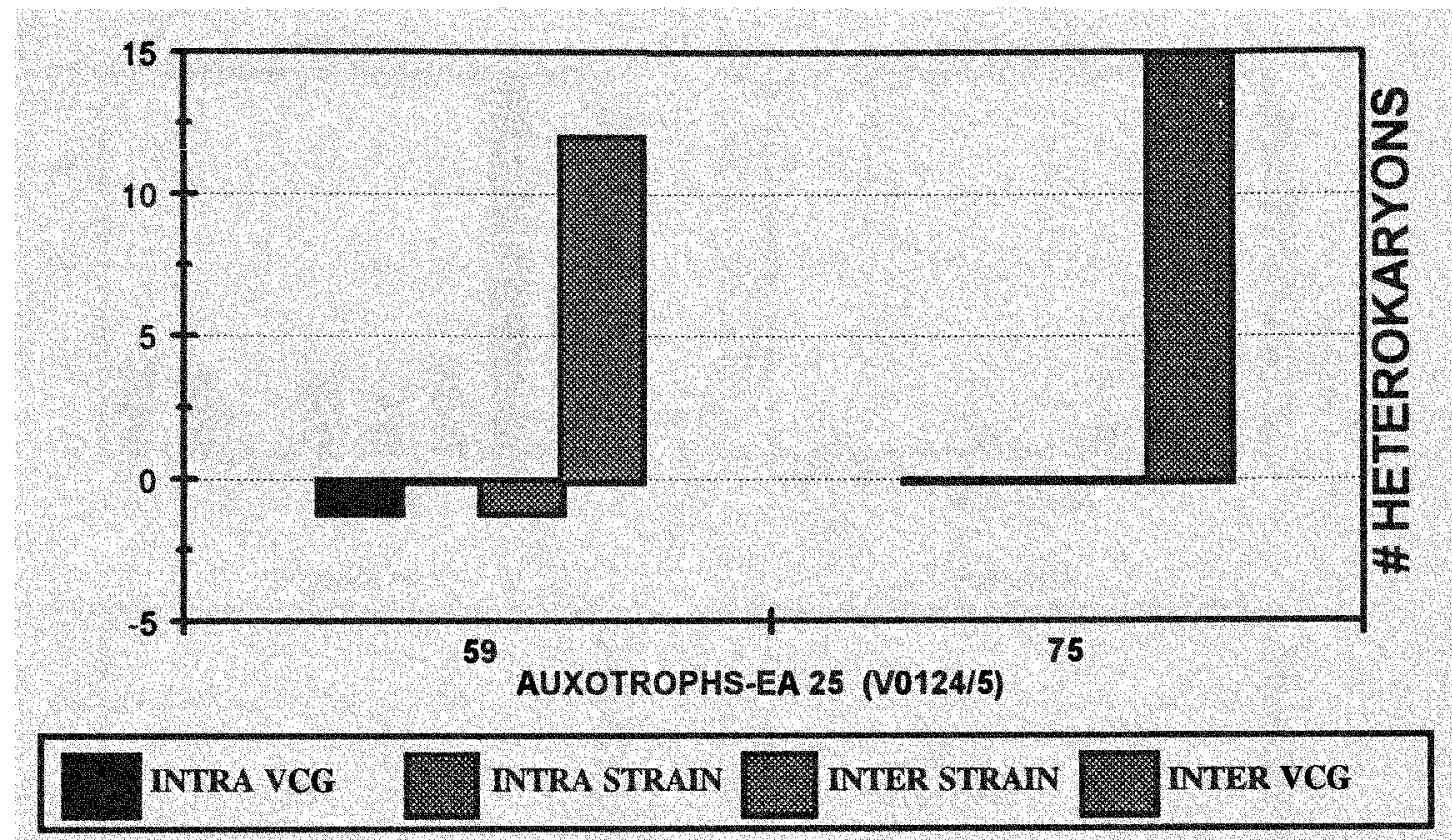

Figure 19 


\section{HET FORMATION-STM3}

V0126
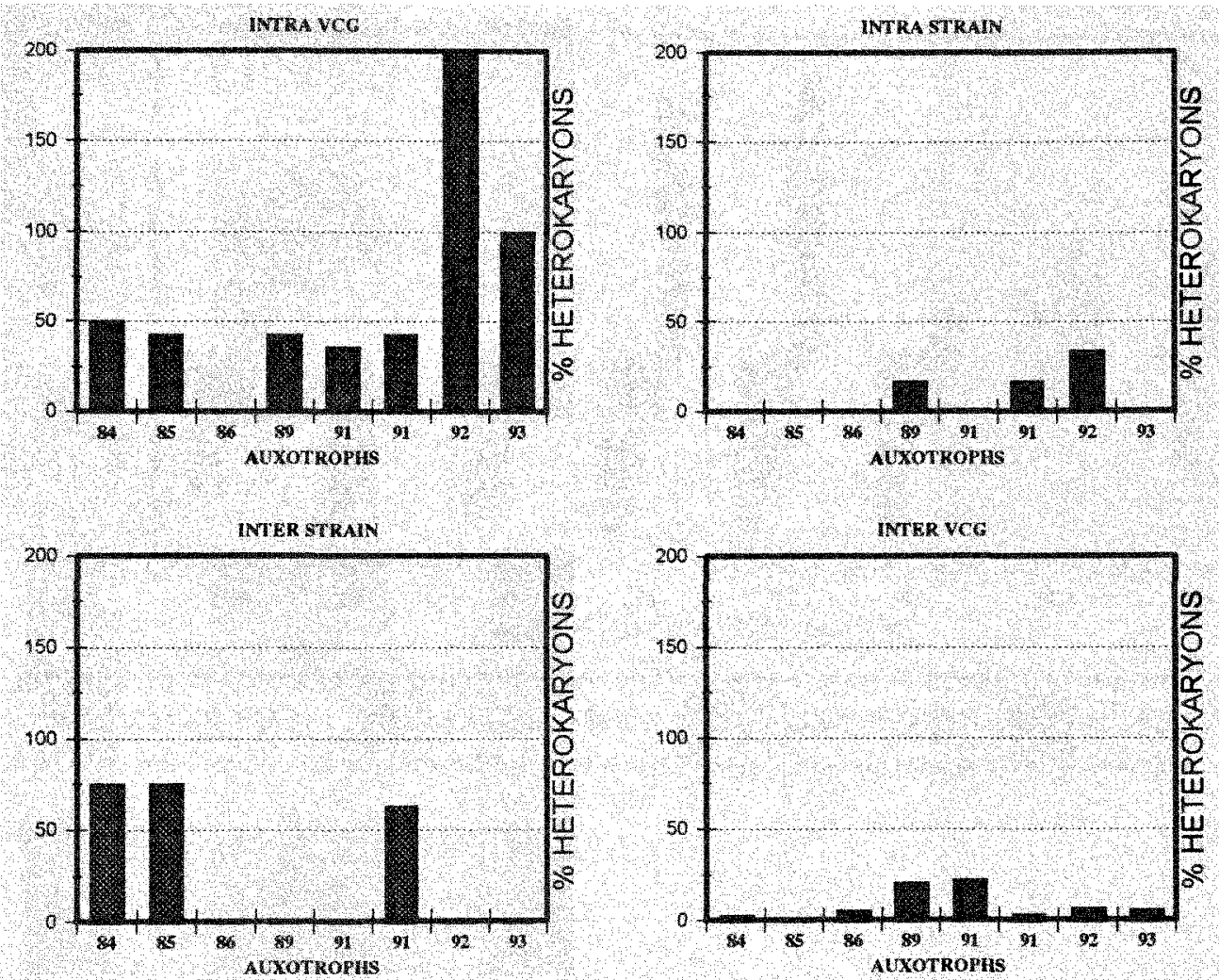

Figure 20 Patterns of heterokaryon formation in strain STM3. The isolates are: \#84 Trp1Met1, \#85 Trp1, \#86 Lys1Ile, \#89 Lys2Arg2, \#91 Lys2Arg2, \#91 Met1Arg3, \#92 Lys1Met1 and \#93 Leu2Lys1 


\section{INTRA VCG INCOMPATIBILITY VS \\ INTER VCG COMPATIBILITY}

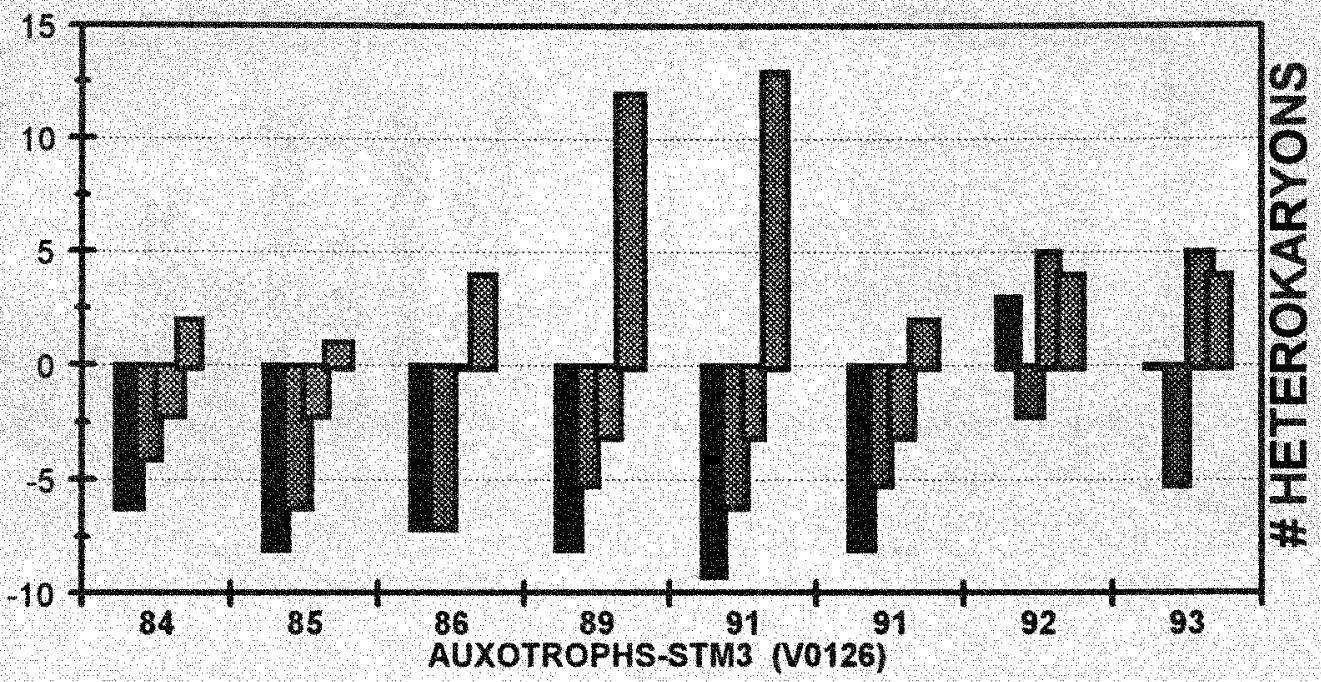

INTRA VCG

INTRA STRAIN

INTER STRAIN

INTER VCG

Figure 21 


\section{HET FORMATION-STB2}

\section{V0126}
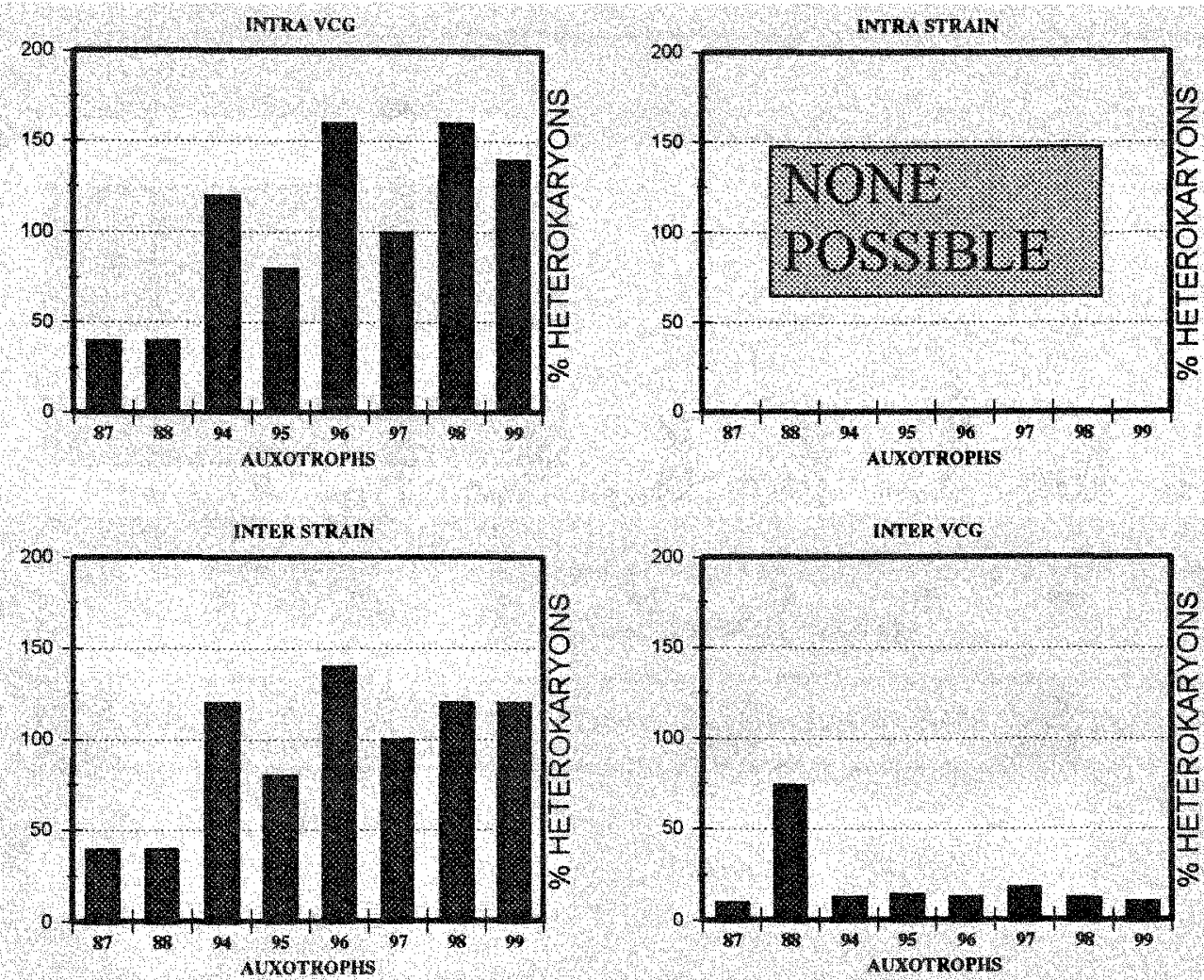

Figure 22 Patterns of heterokaryon formation in strain STB2. The isolates are: \#87 Lys1Tyr1, \#88 Lys1Arg1 B+, \#94 to \#97 Lys1His1, \#98 Lys1 and \#99 Lys1Arg1 


\section{INTRA VCG INCOMPATIBILITY VS \\ INTER VCG COMPATIBILITY}

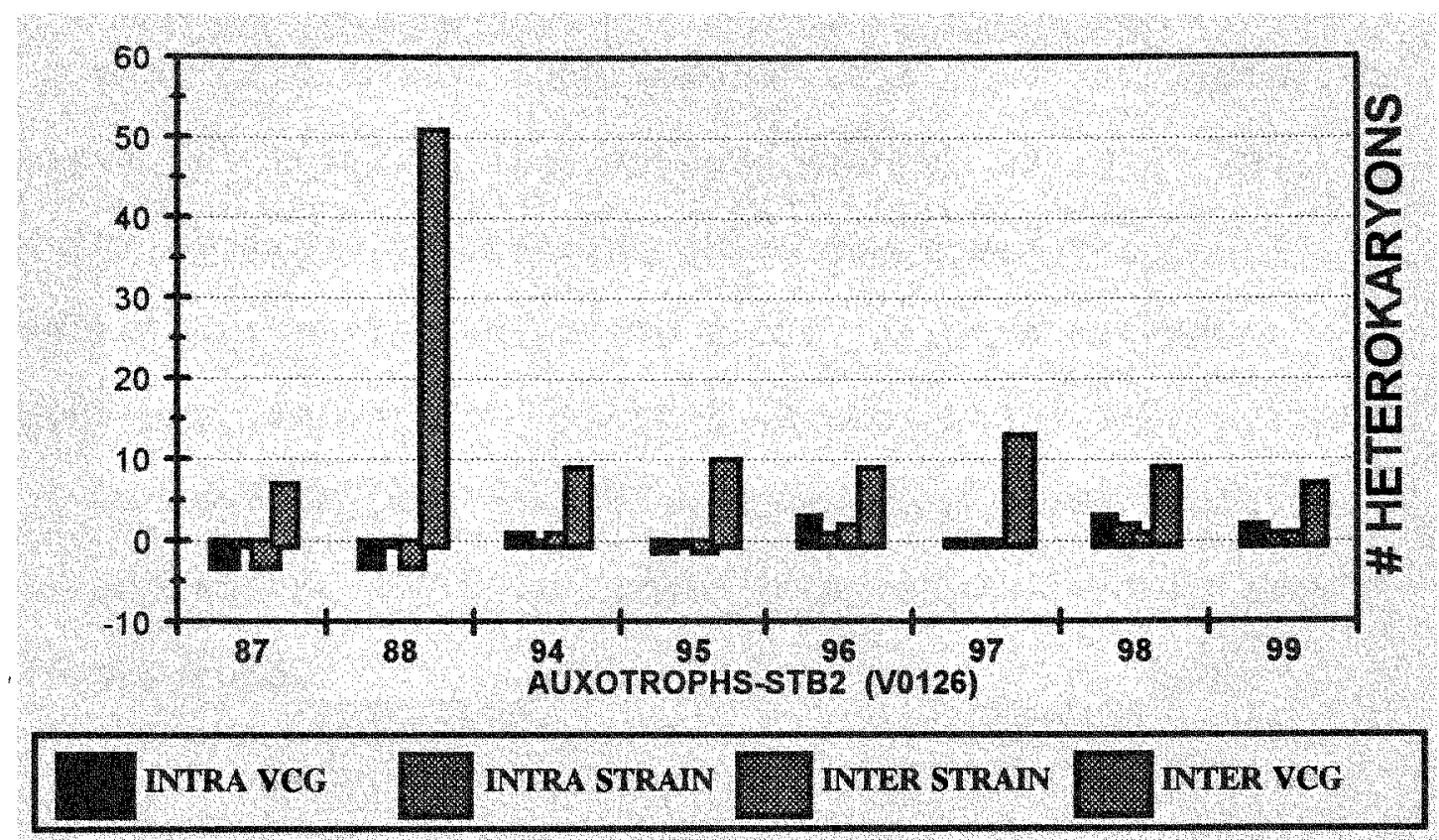

Figure 23 
In strains where the auxotrophs shared an identical nutritional deficiency, pairwise combinations were retested in a mini-grid on MMA supplemented with said deficiency to analyze intra-strain interactions (Tables 13-15). The results obtained in the mini grids confirmed the observations reflected on the first pairings between auxotrophic mutants represented on the Grid. Intra-strain self incompatibility can be observed among some isolates. Poor inbreeders actively formed inter-strain and inter-VCG heterokaryons. 
Table 13 VCG 120: Intra-strain Isolates on MMA + Met

\begin{tabular}{||l|l|l|l|l|l|l||}
\hline AUXOTROPH & NO & 1 & 2 & 3 & 4 & 5 \\
\hline SA8 Met4 Arg1 & 1 & & - & - & + & - \\
\hline SA8 Met4 Arg1 BEN+ & 2 & - & & - & - & - \\
\hline SA8 Met4 Pro & 3 & - & - & & - & - \\
\hline SA8 Uracil & 4 & + & - & - & - & - \\
\hline SA8 Adel & 5 & - & - & - & - & \\
\hline
\end{tabular}

Table 14 VCG 124: Intra-strain Isolates on MMA + Lys

\begin{tabular}{||l|l|l|l|l|l|l|l|l|l|l||}
\hline AUXOTROPH & NO & 1 & 2 & 3 & 4 & 5 & 6 & 7 & 8 & 9 \\
\hline GMB Arg1 & 1 & & + & - & - & + & - & - & - & + \\
\hline GMB Lys1 & 2 & + & & + & + & + & + & + & + & + \\
\hline GMB Met4 & 3 & - & + & & - & + & - & - & + & + \\
\hline GMB Arg2 & 4 & - & + & - & & + & - & - & - & + \\
\hline GMB Lys1BEN+ & 5 & + & + & + & + & & + & + & + & - \\
\hline GMB Ade1 & 6 & - & + & - & - & + & & - & - & - \\
\hline GMB Met4 & 7 & - & + & - & - & + & - & & - & + \\
\hline GMB Lys1 Arg2 & 8 & - & + & + & - & + & - & - & & - \\
\hline GMB Met1 & 9 & + & + & + & + & - & - & + & - & - \\
\hline
\end{tabular}

Table 15 VCG 126: Intra strain Isolates on MMA + Lys

\begin{tabular}{||l|l|l|l|l|l|l||}
\hline AUXOTROPH & NO & 1 & 2 & 3 & 4 & 5 \\
\hline STM3 Lys2 Arg2 & 1 & & - & + & + & + \\
\hline STM3 Lys2 Arg2 & 2 & - & & + & + & - \\
\hline STM3 Met4 Arg3 & 3 & + & + & & + & - \\
\hline STM3 Lys1 Met1 & 4 & + & + & + & & + \\
\hline STM3 Leu2 Lys1 & 5 & + &. &. & + & \\
\hline
\end{tabular}


For organisms genetically identical, we observed great intra VCG variability. If the intra-strain or intra-VCG variability observed is an artifact of UV mutagenesis, it would suggest that het genes are highly mutable and one could anticipate that variability would be seen in the field. Another possibility is that het genes or vic genes are some kind of "hotspot" for UV targeting, which could alter heterokaryon formation, allowing non-self interactions to occur and preventing self interactions from happening. Nonetheless, both, intra-VCG and inter-VCG heterokaryon formation were observed in all tested VCGs (Fig. 24). 


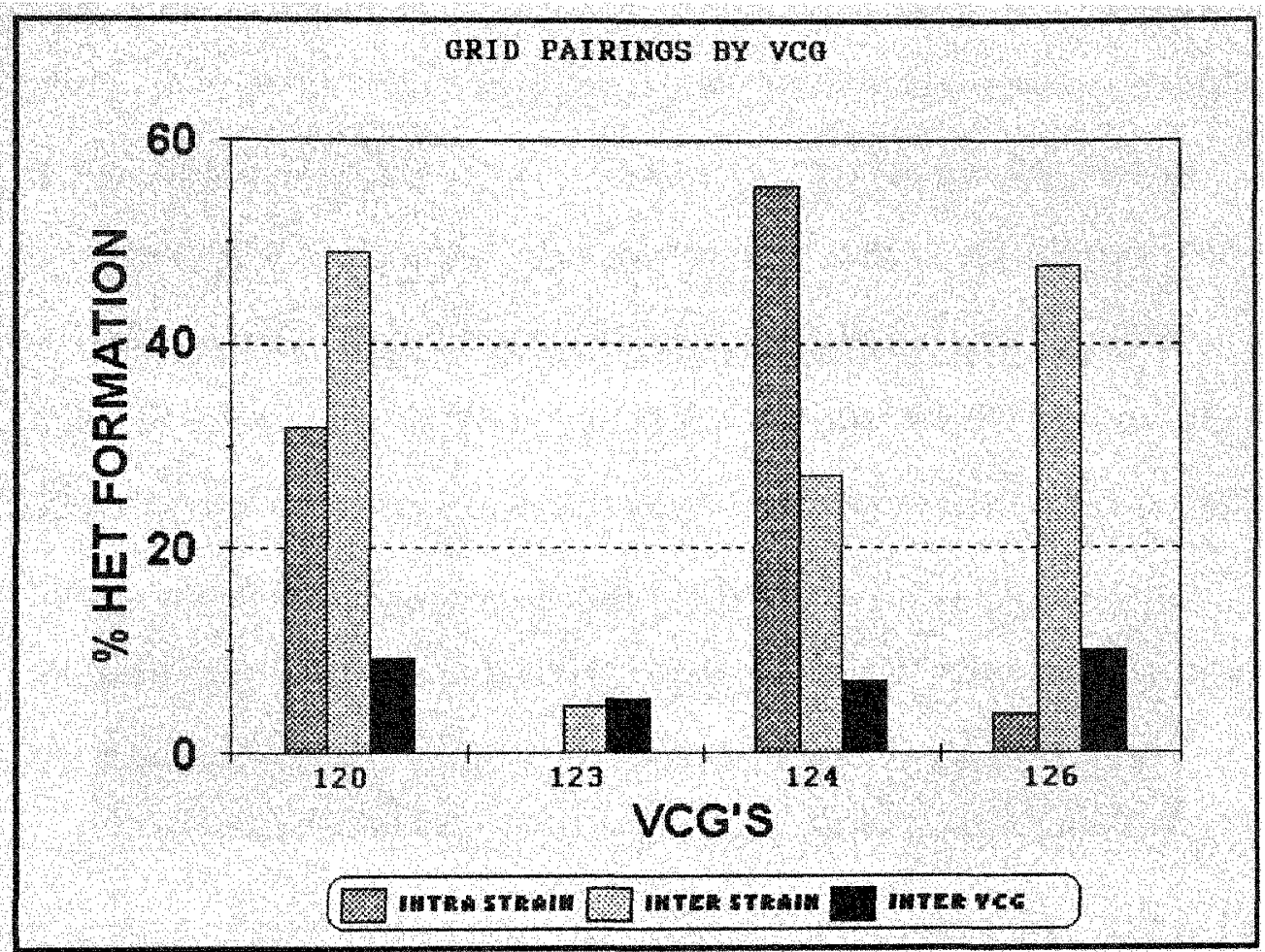

Figure 24 Analysis heterokaryon formation from 10,000 pairings between auxotrophic mutants of Foc. From left to right: VCG 120, 123, 124 and 126 
Although there was no difference in the morphology of heterokaryon formation of intra and inter-VCG pairings, two different heterokaryon morphologies were observed among the pairings: tufty and sparse. Tufty morphology includes aerial mycelia and grows evenly to the edge of the colony. Sparse morphology can be pionnodal or begin tufty and continue sparsely. Radial analysis from both types of heterokaryotic colonies indicated that mycelia continued to grow upon transfer of the plug to MMA.

It has been reported that hyphal tips are multinucleate in Foc (Buxton 1954) but, are vegetative hyphal tips heterokaryotic? To answer this question hyphal tip analysis of heterokaryotic colonies was done. Hyphal tips (Appendix C) were transferred to CMA. Viability of the tips was between 50 and $100 \%$. The emerging colonies were washed for spores and the spore suspension was serially diluted and plated onto CMA. The phenotype of the colonies was determined using the diagnostic test for heterokaryons formed between auxotrophic mutants (Appendix C). Intra-VCG (Table 16) and Inter-VCG (Table 17) heterokaryons were tested. Although the microconidia of some hyphal tips were homogeneous in their phenotype suggestive of a homokaryotic tip (Table 17, Hyphal tips 1, 3 and 5) we found hyphal tips 
whose microconidia were heterogenous(Table 16, hyphal tip 3; Table 17, hyphal tips 2 and 4) suggestive of heterokaryotic tips. We observed two types of putative heterokaryotic tips: one type that grew on diagnostic plates supplemented with the nutritional deficiency of either parent, but incapable of growth on MMA (Table 16, hyphal tips 1, 2, 4 and 5; Table 17, hyphal tips 5) and a second type that grows on all diagnostic plates (Table 16, hyphal tip 3; Table 17, hyphal tips 2 and 4 ). Hyphal tips of the individual parental auxotrophs was homogenous when tested in MMA plates supplemented with their respective deficiencies (Table 16 and Table 17, P1 and P2).

Previously reported radial analysis of heterokaryotic colonies of $F$. moniliforme showed growth on diagnostic plates for both parental phenotypes but reported no growth on MMA (Puhalla 1985), although he reported 100\% homokaryotic tips. 
Table 16 Hyphal Tip Analysis Intra-VCG Het Formation

\begin{tabular}{||l|l|l|l|l||}
\hline HYPHAL TIP & MMA & P1 & P2 & A \\
\hline 1 & $0 / 5$ & $0 / 5$ & $0 / 5$ & $5 / 5$ \\
\hline 2 & $0 / 5$ & $0 / 5$ & $0 / 5$ & $5 / 5$ \\
\hline 3 & $3 / 5$ & $0 / 5$ & $0 / 5$ & $2 / 5$ \\
\hline 4 & $0 / 5$ & $0 / 5$ & $0 / 5$ & $5 / 5$ \\
\hline 5 & $0 / 5$ & $0 / 5$ & $0 / 5$ & $5 / 5$ \\
\hline P1 & $0 / 5$ & $5 / 5$ & $0 / 5$ & $0 / 5$ \\
\hline P2 & $0 / 5$ & $0 / 5$ & $5 / 5$ & $0 / 5$ \\
\hline
\end{tabular}

P1= STM3 Lys2 Met1 P2= STB2 Lys1 His1

Table 17 Hyphal Tip Analysis Inter-VCG Het Formation

\begin{tabular}{||l|l|l|l|l|}
\hline HYPHAL TIP & MMA & P1 & P2 & A \\
\hline 1 & $0 / 5$ & $0 / 5$ & $5 / 5$ & $0 / 5$ \\
\hline 2 & $2 / 5$ & $3 / 5$ & $0 / 5$ & $0 / 5$ \\
\hline 3 & $0 / 5$ & $0 / 5$ & $5 / 5$ & $0 / 5$ \\
\hline 4 & $1 / 5$ & $0 / 5$ & $4 / 5$ & $0 / 5$ \\
\hline 5 & $0 / 5$ & $0 / 5$ & $0 / 5$ & $5 / 5$ \\
\hline P1 & $0 / 5$ & $5 / 5$ & $0 / 5$ & $0 / 5$ \\
\hline P2 & $0 / 5$ & $0 / 5$ & $5 / 5$ & $0 / 5$ \\
\hline
\end{tabular}

P1= STB2 Lys1 His1 Ade P2 = SA8 Met4 Pro1 
To determine if the out crossing phenomena occurred between genetically distant isolates and to assess if interVCG heterokaryon formation was observed using unmutagenized wild type strains, we forced heterokaryon formation using auxotrophic benomyl resistant mutants (Wild Type Testers (WTT)) (Appendix C) .

Benomyl is a powerful fungicide used in the control of fungal plant pathogens including Fusarium oxysporum f.sp. cubense. Benomyl attacks tubulin impairing microtubule formation and preventing mitosis.

Titrations using different concentrations of benomyl in CMA plates were used to test the sensitivity of the wild types (Appendix A). The growth of wild type strains of FoC and the tested formae speciales was inhibited at benomyl concentrations between 2 and $3 \mu \mathrm{g} / \mathrm{ml}$. Two species of Fusarium, Fusarium solani and Fusarium solani pisi showed natural resistance to the fungicide. Growth inhibition for these two species was observed at concentrations higher than $50 \mu \mathrm{g} / \mathrm{ml}$. Our Benomyl resistant mutants are capable of withstanding benomyl concentrations between 10 and 100 $\mu \mathrm{g} / \mathrm{ml}$. 
Pairwise combinations were conducted between the wild type testers and wild types in the 15 known VCGs of Foc. The wild type testers were also paired against four different formae speciales known to be genetically more distant than intra-forma strains (Kistler 1991) (Appendix A). Pairings were made using the double pick method and six wild type testers representing VCGs 0120, two wild type testers representing VCG 0124 and one wild type tester representing VCG 0126 (Table 18).

The results are reported as a ratio. The numerator reflects the number of heterokaryons formed between the wild type tester and the different strains. The denominator reflects the total possible number of heterokaryons formed (number of wild type testers times the number of tested strains). The parental phenotypes were double-picked in the plates against themselves as control. No growth was detected in the area stabbed with either the wild types or the wild type testers. Growth in the pairing areas was detected 7-10 days after the pairings were done. The morphology of the emerging colonies is compact and growth is slower, probably due to the benomyl. Normal growth was resumed upon transfer of the colonies to CMA. Five trials were done per pairwise combination. 
Table 18 Heterokaryon Formation using wild Type Testers

\begin{tabular}{|c|c|c|c|c|}
\hline $\begin{array}{l}\text { WILD TYPES } \\
\text { (VCG) }\end{array}$ & $\begin{array}{l}\text { TESTED } \\
\text { STRAINS } \\
\end{array}$ & $\begin{array}{c}\text { WTT-V0120 } \\
(6)\end{array}$ & $\begin{array}{c}\text { WTT-V0124 } \\
\text { (2) } \\
\end{array}$ & $\begin{array}{c}\text { WTT-V0126 } \\
\text { (1) }\end{array}$ \\
\hline 0120 & 12 & $47 / 72$ & $8 / 24$ & $3 / 12$ \\
\hline 0121 & 2 & $8 / 12$ & $0 / 4$ & $0 / 2$ \\
\hline 0122 & 3 & $12 / 18$ & $2 / 6$ & $1 / 3$ \\
\hline 0123 & 1 & $3 / 6$ & $2 / 2$ & $0 / 1$ \\
\hline 0124 & 9 & $38 / 54$ & $9 / 18$ & $4 / 9$ \\
\hline $0124 / 5$ & 3 & $13 / 18$ & $4 / 6$ & $0 / 3$ \\
\hline 0125 & 2 & $7 / 12$ & $3 / 4$ & $1 / 2$ \\
\hline 0126 & 4 & $10 / 24$ & $4 / 8$ & $2 / 4$ \\
\hline 0128 & 2 & $5 / 12$ & $1 / 4$ & $1 / 2$ \\
\hline 0129 & 2 & $6 / 12$ & $2 / 4$ & $1 / 2$ \\
\hline 01210 & 2 & $6 / 12$ & $1 / 4$ & $1 / 2$ \\
\hline 01211 & 2 & $8 / 12$ & $2 / 4$ & $1 / 2$ \\
\hline 01212 & 2 & $9 / 12$ & $2 / 4$ & $0 / 2$ \\
\hline 01213 & 1 & $4 / 6$ & $1 / 2$ & $0 / 1$ \\
\hline 01214 & 2 & $6 / 12$ & $2 / 4$ & $0 / 2$ \\
\hline \multicolumn{5}{|l|}{$\begin{array}{l}\text { FORMAE } \\
\text { SPECIALES } \\
\end{array}$} \\
\hline LYCOPERSICI & 3 & $7 / 18$ & $3 / 6$ & $2 / 3$ \\
\hline RAPHANI & 1 & $1 / 18$ & $1 / 2$ & $0 / 1$ \\
\hline PISI & 1 & $0 / 18$ & $1 / 2$ & $1 / 1$ \\
\hline CONGLUTINANS & 1 & $0 / 18$ & $1 / 2$ & $0 / 1$ \\
\hline
\end{tabular}


Similarities were observed between the heterokaryons formed using two auxotrophic mutants and those heterokaryons formed between the wild type testers and wild type strains. First of all, not all expected intra-VCG pairings occurred while inter-VCG heterokaryon formation was observed between the wild type testers in the three tested VCGs and all 15 VCGS of FOC.

For example, wild type testers in VCG 0120 paired with 65\% of the strains represented in that VCG while forming heterokaryons with $40 \%$ to $70 \%$ of inter VCG strains. Similar results were obtained for wild type testers in VCG 0124 and 0126. The frequency of heterokaryon formation varied between strains and two of the testers (STH1 and JCB1) paired almost exclusively within their VCG, although some outcrossing was detected.

Due to the genetic distance between the isolates, the inter-forma heterokaryon formation was unexpected. Random analysis of the progeny on diagnostic plates indicated that inter-forma heterokaryons appear to be stable, as several generations of spores gave rise to prototrophic growth on MMB plates (Appendix C). Of particular interest was the pairing between GMB Lys1 Bt and Fusarium oxysporum forma specialis lycopersici as prototrophic growth was detected 
when plating $10^{3}$ spores of the heterokaryon on MMB, suggestive of hybrid formation.

Several observations are worth mentioning from the analysis of pairings presented in the grid and the wild type testers pairings: first, there is great intra-VCG variability among the isolates and we can narrow this variability down to the isolate level; second, isolates showing altered heterokaryon formation were identified in several strains such as 22425, STB2 and GMB.

A skewed number of colonies representing one parental phenotype over the other was always recovered, but the number of spores recovered from each "parent" seemed to be dependent on the pairing interaction and not on the individual isolates been paired.

More important, though, is the fact that inter-VCG and inter-forma heterokaryon formation was confirmed suggesting that the barriers imposed by the genetic distance of Vegetative Compatibility Groups and formae speciales are not stringent enough to prevent heterokaryosis. Isolates capable of heterokaryon formation via hyphal anastomosis with isolates of different VCGs were observed in all tested VCGs .

If we consider the parasexual cycle as a means to generate genetic diversity in the field, greater diversity 
could be achieved by pairing with organisms more distantly related.

Indeed, the prototrophic growth observed on diagnostic analysis of microconidia from heterokaryons between auxotrophic mutants was suggestive of putative hybrid formation. A closer analysis was needed to determine if karyogamy, the second stage of the parasexual cycle occurred in FOC.

\section{Hybrid Formation in Foc}

Although not all heterokaryotic events lead to karyogamy, hybrid formation is dependent on heterokaryosis happening first. Several outcomes are expected from screening microconidia of heterokaryotic cells in asexual organisms: spores representing both parental phenotypes or prototrophic spores suggestive of karyogamy (fusion of the different nuclei), giving rise to diploid formation. A conservative approach was adopted by identifying as hybrids (putative diploids) those propagules from heterokaryons between auxotrophic mutants capable of prototrophic growth on MMA.

From the pairings between auxotrophic mutants, randomly selected heterokaryons were analyzed and their microconidia tested on diagnostic plates to screen for hybrid formation. 
Microconidia analysis is a useful tool to screen for hybrid formation since microconidia are uninucleate. Microconidia of auxotrophs cannot survive on unaugmented media, as the spores try to germinate and die. Therefore, prototrophic growth of microconidia on MMA at low density is suggestive of the nuclear fusion characteristic of diploid formation.

Hybrids were observed both on intra-VCG (Table 19) and inter-VCG (Table 20) pairings. The number of hybrids is presented as the number of hybrids found per number of heterokaryons screened. Each hybrid comes from a single heterokaryotic event. Although expected, the number of putative hybrids was low. 
Table 19 Intra VCG Hybrid Formation

\begin{tabular}{||l|l|l|l|l||}
\hline PAIRINGS & $\begin{array}{l}\text { \# OF } \\
\text { HYBRIDS }\end{array}$ & $\begin{array}{l}\text { NUMBER OF } \\
\text { SECTORS FM } \\
\text { HYBRIDS }\end{array}$ & SECTOR & GENOTYPE \\
\hline K1R1(P1) X & $9 / 100$ & $5 / 9$ & S1 & P1 \\
M1R3 (P2) & & & S2 & P1 \\
& & S3 & P2 \\
& & S4 & A \\
& \multirow{2}{*}{$3 / 50$} & $5 / 3$ & S5 & S1 \\
M1R3(P1) X & & & S2 & P2 \\
& & & S3 & H \\
& & & S4 & S5 \\
\hline
\end{tabular}

LEGEND: $K=$ Lys, $R=$ Arg, M=Met, L=Leu, Ade=Adenosine, $\mathrm{B}+=$ Benomyl resistant, Pyr=Pyridoxine; Genotypes: $\mathrm{Pl}$ =Parent 1. P2=Parent 2, H=Hybrid, A=Altered 
Table 20 Inter VCG Hybrid Formation

\begin{tabular}{|c|c|c|c|c|}
\hline PAIRINGS & $\begin{array}{l}\text { \# OF } \\
\text { HYBRIDS }\end{array}$ & $\begin{array}{l}\text { NUMBER OF } \\
\text { SECTORS FM } \\
\text { HYBRIDS }\end{array}$ & SECTOR & GENOTYPE \\
\hline $\begin{array}{l}\mathrm{K} 1 \mathrm{R} 1 \mathrm{~B}+(\mathrm{P} 1) \mathrm{X} \\
\mathrm{M} 4(\mathrm{P} 2)\end{array}$ & $1 / 50$ & $1 / 1$ & s1 & $\mathrm{H}$ \\
\hline $\begin{array}{l}\text { Pyr1K2(P1) X } \\
\text { K1R1B + (P2) }\end{array}$ & $1 / 50^{*}$ & $8 / 1$ & $\begin{array}{l}\text { S1 } \\
\text { S2 } \\
\text { S3 } \\
\text { S4 } \\
\text { S5 } \\
\text { S6 } \\
\text { S7 } \\
\text { S8 }\end{array}$ & $\begin{array}{l}\mathrm{P} 1 \\
\mathrm{P} 2 \\
\mathrm{P} 2 \\
\mathrm{H} \\
\mathrm{H} \\
\mathrm{H} \\
\mathrm{H} \\
\mathrm{A}\end{array}$ \\
\hline $\begin{array}{l}\text { R3Ade1B + (P1) X } \\
\text { K1R1B + (P2) }\end{array}$ & $1 / 50^{*}$ & $4 / 1$ & $\begin{array}{l}\text { S1 } \\
\text { S2 } \\
\text { S3 } \\
\text { S4 } \\
\end{array}$ & $\begin{array}{l}\mathrm{P} 1 \\
\mathrm{P} 2 \\
\mathrm{P} 2 \\
\mathrm{H}\end{array}$ \\
\hline $\begin{array}{l}\text { K1H1(P1) X } \\
\text { M4R3 (P2) }\end{array}$ & $1 / 50 *$ & $1 / 1$ & S1 & $\mathrm{H}$ \\
\hline
\end{tabular}

Legend: $K=L y s, R=A r g, M=M e t, L=L e u$, Ade=Adenosine, $\mathrm{B}+=$ Benomyl resistant, Pyr=Pyridoxine; Genotype: $\mathrm{P} 1=$ Parent 1, P2=Parent 2, H=Hybrid, A=Altered 
Looking to increase the number of detected hybrids, and following the principle behind auxotroph filter enhancement, we designed a hybrid enhancement technique that increased the number of screened hybrid colonies (Appendix C). Hybrid formation was observed as early as two weeks. The heterokaryotic mycelial plugs were transferred to MMA plates and screened for continued growth. Spores were harvested from the transferred plug and analyzed in diagnostic plates. Recovery of both parental phenotypes confirmed the initial heterokaryotic event.

However, an increased number of prototrophic colonies emerged in the MMA plates. Although several prototrophic colonies came from the pairings on each tested tube, we identified the pool of hybrids coming from a single tube as a single putative hybrid event("*" Table 20). By plating the progeny of heterokaryotic colonies on MMA using dilutions of $10^{3}$ spores or less we are strongly selecting for hybrid progeny. Hybrid enhancement was $33-50 \%$ effective in detecting an increased number of hybrids (Table 21).

Table 21 Hybrid Enhancement

\begin{tabular}{||l|l|l|c|}
\hline $\begin{array}{l}\text { K1H1AdeB+ X } \\
\text { M4PrOB+ }\end{array}$ & $\begin{array}{l}\text { \# INDIVIDUAL } \\
\text { HETEROKARYONS }\end{array}$ & \# HYBRIDS & $\%$ \\
\hline TRIAL 1 & 45 & $15 / 45$ & $33 \%$ \\
\hline TRIAL 2 & 44 & $22 / 44$ & $50 \%$ \\
\hline TRIAL 3 & 50 & $23 / 50$ & $46 \%$ \\
\hline
\end{tabular}


The average number of microconidia from heterokaryons showing a hybrid phenotype also increased (Table 22).

Table 22 Diagnostic Test Enhanced Microconidia

\begin{tabular}{||l|l|l|l|l|l|}
\hline PAIRINGS & P1 & P2 & D & A & CONTROL \\
\hline $\begin{array}{l}\text { K1H1Ade1B + X } \\
\text { M4 Prol B }+\end{array}$ & 259 & 458 & 465 & 33 & 1215 \\
\hline
\end{tabular}

Genotypes: P1=Parent 1, P2=Parent 2, D=Hybrid, A=Altered

The final test of hybrid formation comes from hyphal tip analysis. Prototrophic colonies on MMA were transferred to $1.5 \%$ water agar thinly plated. The water agar allows for very sparse growth facilitating the removal of the hyphal tips (Appendix C). Tips were transferred to MMA and CMA plates for further analysis. From the viable tips, spores were collected, plated on CMA and diagnosed (Table 23). Twenty-five percent of the hyphal tips transferred to MMA grew.

\section{TABLE 23 Hyphal Tip Analysis}

\begin{tabular}{||l|l|l|l|l|l||}
\hline HYBRID & VIABLE & $\mathrm{P} 1$ & $\mathrm{P} 2$ & HYBRID & SECTOR \\
\hline $\begin{array}{c}\text { Lys1Arg1 B+ } \\
\text { Met4 }\end{array}$ & $12 / 15$ & $6 / 12$ & $2 / 12$ & $4 / 12$ & $\begin{array}{r}2 / 4: \mathrm{S} 1 \mathrm{P} 1 \\
\text { S2*A }\end{array}$ \\
\hline $\begin{array}{c}\text { Pyr1 Lys2 } \\
\text { Lys1Arg1 B+ }\end{array}$ & $16 / 20$ & $10 / 16$ & $2 / 16$ & $3 / 16$ & $1 / 3:$ S1 P1 \\
\hline $\begin{array}{c}\text { Arg3Ade1B+ } \\
\text { Lys1Arg1 B+ }\end{array}$ & $9 / 20$ & $2 / 9$ & $1 / 9$ & $6 / 9$ & $1 / 6:$ S1 A \\
\hline
\end{tabular}

*sector 1 had a parent 1 phenotype while sector 2 had parent 1 phenotype and parent 2 mitochondrial type. 


\section{Altered parental phenotypes}

Analysis of sectors from hybrids transferred to unselective media identified sectors showing a recombinant phenotype (Table 22). Some sectors grew only on CMA plates but not on the other diagnostic plates or where the auxotrophs shared a common nutritional deficiency, growth was observed on diagnostic plates for parent 1 and parent 2 genotype but not on MMA. Further analysis of those colonies on MMA plates supplemented with the combined parental deficiencies indicated that the following genotypes were recovered:

\section{Intra-VCG}

Pairing sectors Showing Altered Genotype $\mathrm{K} 1+\mathrm{R} 1 \mathrm{~K}-\mathrm{M}+\mathrm{R}-, \mathrm{K}-\mathrm{M}-\mathrm{R}-$ + M1 R3

\section{Inter-VCG}

Pairing Sectors Showing Altered Genotype Pyr1+ K2B- Pyr-K-R$+\mathrm{R} I \mathrm{~K} 1 \mathrm{~B}+$

Analysis of the progeny of a hybrid formed between two benomyl resistant parents identified some prototrophic colonies (a hybrid genotype) that grew on regular MMA plates but were unable to grow on MMA plates supplemented with benomyl.

Pairing

$\frac{\mathrm{K} 1 \mathrm{H} 1+\mathrm{Ade}+\mathrm{B}+}{++\mathrm{M} 4+\mathrm{P} \mathrm{B}+}$

Nuclear Genotype

$$
\mathrm{K}+\mathrm{H}+\mathrm{M}+\mathrm{Ade}+\mathrm{P}+\mathrm{B}-
$$


of greater significance was the fact that two hyphal tips of hybrids sectored when transferred to CMA. In collaboration with Naomi D'Alessio, single strand Conformation Polymorphism (Walsh 1995) analysis was conducted on the sectors (Table 23, K1R1 b+ x M4). The nuclear phenotype of one of the sectors represented the nuclear markers of parent 1 (although benomyl sensitive) when analyzed using the diagnostic test (Appendix $C$ ), but the mitochondrial type of parent two under the SSCP test (personal communication).

Pairing

$\frac{\mathrm{K} 1+\mathrm{R} 1 \mathrm{~B}+(\mathrm{MT} I V)}{+\mathrm{M} 4+\mathrm{B}-(\mathrm{MT} \mathrm{I})}$
Altered Parental Genotype $\mathrm{K}-\mathrm{R}-\mathrm{B}-\mathrm{MT}$ I

Other sectors (Table 23, R3Ade1 $\mathrm{B}+\mathrm{X} \mathrm{R} 1 \mathrm{~K} 1 \mathrm{~B}+$ ) were also analyzed under standard diagnostic tests (Appendix C). One sector grew on diagnostic plates for both parent 1 and parent 2 but not on MMA plates. When screened on MMA supplemented with combinations of the amino acids representing the parental deficiencies we identified the sector as Arg negative.

Pairing

$\mathrm{R} 3+\mathrm{Ade1} \mathrm{B}+$

\section{Altered Parental Genotype}

R- 
Haploidization, the Final stage of the Parasexual cycle

In addition to the sectors with an altered parental genotype, other sectors were detected growing out of hybrid colonies (Table 24). Diagnostic tests (Appendix C) identified the sectors as having either a parental phenotype or a hybrid phenotype, providing evidence for haploidization, the last stage of the parasexual cycle (Pontecorvo 1953).

We suspect aneuploidy could be responsible for the observed high frequency of a particular auxotrophy generated in FOC (Appendix D), since the effect of the damage caused by UV radiation on an isolate having more than one copy of a chromosome may be masked by the extra copy. Another sign of aneuploidy could be the resistance of some strains to generate auxotrophs via UV mutagenesis such as A2 in VCG 0120, Maca and Bluggoe in VCG 0124, and STM1 in VCG 0126.

Regardless of the strains, pairings between auxotrophic mutants of Foc yield balance heterokaryons and stable hybrid formation when grown on selective media.

No induction of segregants was observed by using haploidizing agents when tested under selective pressure on unsupplemented MMA. However, once the colonies were removed from selective pressure by transferring plugs to CMA, hybrid colonies sectored readily. Analysis of those sectors identified segregants showing a hybrid phenotype, parental 
phenotypes and recombinant phenotypes, consistent with the expectations of hybrid breakdown products (Pontecorvo 1953). 
Table 24 Analysis of sectors

\begin{tabular}{|c|c|c|}
\hline PAIRINGS & SECTOR & GENOTYPE \\
\hline \multicolumn{3}{|l|}{ INTRA-VCG } \\
\hline$\frac{\mathrm{K} 1+\mathrm{R} 1}{+\mathrm{M} 1 \mathrm{R} 3}$ & $\begin{array}{l}\text { S1 } \\
\text { S2 } \\
\text { S3 }\end{array}$ & $\begin{array}{l}\text { P1: K-R- } \\
\text { P1: K-R- } \\
\text { P2: M-R- }\end{array}$ \\
\hline$\frac{\mathrm{M} 1+\mathrm{R} 3+}{+\mathrm{L} 1+\mathrm{K} 2}$ & $\begin{array}{l}\text { S1 } \\
\text { S2 } \\
\text { S3 } \\
\text { S4 } \\
\text { S5 } \\
\end{array}$ & $\begin{array}{l}\text { P1: M-R- } \\
\text { P2: L-K- } \\
\text { H: } M+R+L+K+ \\
H: M+R+L+K+ \\
\text { H: } M+R+L+K+\end{array}$ \\
\hline \multicolumn{3}{|l|}{$\begin{array}{l}\text { INTER-VCG } \\
(120 \times 126) \\
\end{array}$} \\
\hline$\frac{\mathrm{K} 1+\mathrm{R} 1 \mathrm{~B}+}{+\mathrm{M} 4+\mathrm{B}-}$ & S1 & $\mathrm{H}: \mathrm{K}+\mathrm{R}+\mathrm{M}+\mathrm{B}+$ \\
\hline$\frac{\text { Pyrl }+K_{2} B-}{+\mathrm{R} 1 \mathrm{~K} 1 \mathrm{~B}+}$ & $\begin{array}{l}\text { S1 } \\
\text { S2 } \\
\text { S3 } \\
\text { S4 } \\
\text { S5 } \\
\text { S6 } \\
\text { S7 }\end{array}$ & $\begin{array}{l}\text { P1: Pyr-K-B- } \\
\text { P2: R-K-B+ } \\
\text { P2: R-K-B+ } \\
\text { H: } \mathrm{Pyr}+\mathrm{K}+\mathrm{R}+\mathrm{B}+ \\
\mathrm{H}: \mathrm{Pyr}+\mathrm{K}+\mathrm{R}+\mathrm{B}- \\
\mathrm{H}: \mathrm{Pyr}+\mathrm{K}+\mathrm{R}+\mathrm{B}+ \\
\mathrm{H}: \mathrm{Pyr}+\mathrm{K}+\mathrm{R}+\mathrm{B}+\end{array}$ \\
\hline$\frac{\mathrm{R} 3 \mathrm{Ade} 1++\mathrm{B}+}{++\mathrm{K} 1 \mathrm{R} 1 \mathrm{~B}+}$ & $\begin{array}{l}\text { S1 } \\
\text { S2 } \\
\text { S3 } \\
\text { S4 } \\
\end{array}$ & $\begin{array}{l}\text { P1: R-Ade-B+ } \\
\text { P2: K-R-B }+ \\
\text { P2: K-R-B } \\
\text { H: R+Ade+K+B+ }\end{array}$ \\
\hline$\frac{\mathrm{K} 1 \mathrm{H} 1+t}{++\mathrm{M} 4 \mathrm{R} 3}$ & S1 & $\mathrm{H}: \mathbf{K}+\mathrm{H}+\mathrm{M}+\mathrm{R}+$ \\
\hline
\end{tabular}




\section{CONCLUSION}

With the emergence of new pathogenic races among asexual fungal pathogens of important agricultural crops worldwide, the importance of understanding parasexual processes has become apparent. To study this phenomenon we used the asexual phytopathogen Fusarium oxysporum f.sp. cubense as a model system. Not only were we able to ascertain that all stages of the parasexual cycle occurred in Foc but analysis of the process allowed us to postulate some interesting ideas regarding fungal interactions. The first step of the cycle deals with heterokaryon formation. If we consider that genetic identical pathogenic strains of Foc are grouped in different vegetative compatibility groups based on their ability to form heterokaryons via hyphal anastomosis, one should expect to see a high incidence of intra VCG heterokaryon formation and a nonexistent record of inter-VCG het formation. Analysis of 10,000 pairwise combinations between 100 auxotrophic mutants of Foc disclosed a different story.

The most important outcome was the discovery that heterokaryon formation in Foc was not hindered by race, strain, Vegetative compatibility Group or formae speciales, suggesting that the distances imposed by current groupings are not stringent enough to prevent heterokaryon formation via hyphal anastomosis, which antagonizes with the current 
view of Vegetative compatibility Group assignments. We suggest a classification to identify fungal interactions in FOC based on anastomosis groups and not on VCGs.

There is no difference between intra- and inter-VCG heterokaryon formation, nor does the method used to force heterokaryosis affects the process. The frequency and the morphology of the heterokaryons formed seems to be governed by the interactions of the individual isolates being paired. The heterogeneity found on analysis of microconidia of hyphal tips of heterokaryons is suggestive of the multinucleate, heterokaryotic nature of the hyphal tips.

Two different heterokaryon morphologies were identified: tufty and sparse. Contrary to expectations, radial analysis of sparse heterokaryons revealed that both parental phenotypes were present in a cell but were incapable of sustained growth on unsupplemented MMA plates.

A possible explanation could be that both types of nuclei are present in the germ tube separated by the septa, just behind the extension front; close enough to allow cross-feeding to promote growth in plates screening for P1 and P2 phenotypes but not strong enough to allow prototrophic growth on MMA plates.

Another possibility takes us back to the concept of partial heterokaryon incompatibility (Coenen 1994). Perhaps, as seen in Aspergillus, partial-het genes are 
present in Foc preventing healthy development of heterokaryons between incompatible strains but not stringent enough to prevent horizontal transfer of genetic information. Partial-het genes could explain why genetically distant isolates could bypass incompatibility barriers and anastomose under unfavorable conditions. Tufty heterokaryon formation could be evidence of complementary, compatible interactions. The incidence of tufty or sparse seems to be dependent on the individual isolates being paired although there seems to be a higher incidence of sparse heterokaryon formation when isolates of SA8 (Race 4, VCG 0120) are paired against isolates in other races, regardless of VCG.

We have developed two methods to increase the efficiency of forcing heterokaryon formation and to screen hybrid formation.

The following can be stated from the analysis of these prototrophic colonies: heterokaryon formation in Foc seems to be a stable process as no segregation was observed on heterokaryons, even when transferred to unselective media. Hybrids on the other hand, are unstable and sectored readily when removed from selective pressure. Recovery of sectors showing altered parental genotypes (putative somatic recombination) suggests that the parasexual cycle could be a 
tool used by asexual organisms to generate genetic diversity and perhaps new pathogenic races.

Sectors showing a parental phenotype provided evidence of haploidization, the final step of the parasexual cycle. If we consider that microconidia in FOC is uninucleate, the fact that sectors showing both parental genotypes were recovered from hybrid colonies is an indication that both genomes were present in the nucleus.

In summary, all stages of the parasexual cycle occurred in Foc. Heterokaryon formation is not impaired by race or strain interactions. Moreover, the barriers imposed by the concepts of vegetative compatibility or genetic distances imposed by host specificity are not stringent enough to prevent heterokaryon formation in FOC. This suggests that perhaps inter-specific heterokaryon formation is possible, allowing for greater genetic diversity.

Ultimately, understanding parasexual processes can be an important tool in pathogen control management both in agriculture and clinical mycology. Biotechnological processes such as biodegradation or fermentation may also benefit from the parasexual cycle by moving important genes between organisms via natural biological processes. 
Ainsworth, AM and Rayner, ADM (1986) Responses of living hyphae associated with self and non-self fusions in the basidiomycete, Phanerochaete velutina. Journal of General Microbiology, 132: 191-201

Ainsworth, AM and Rayner, ADM (1989) Hyphal and mycelial responses associated with genetic exchange within and between species of the basidiomycete genus stereum. Journal of General Microbiology, 135: 1643-1659

Aker williams, D, Xiang, X, Morris, NR (1995) An alpha tubulin mutation suppresses nuclear migration mutations in Aspergillus nidulans. Genetics 141: 1287-1298

Amoah, BK, Rezanoor, HN, Nicholson, P, MacDonald, MV (1995) Variation in the Fusarium section Liseola pathogenicity and genetic studies of isolates of Fusarium moniliforme Sheldon from different hosts in Ghana. Plant Pathology $44(93): 563-572$

Anwar, MM, croft JH, Dales RBG (1993) Analysis of heterokaryon incompatibility between heterokaryoncompatibility $\mathrm{H}-\mathrm{C}$ groups $\mathrm{R}$ and $\mathrm{GL}$ provides evidence that at least eight het loci control somatic incompatibility in Aspergillus nidulans. Journal of General Microbiology 139(7): 1599-1603

Anderson, JB and Kohn, LM (1995) clonality in soilborne, Plant-Pathogenic fungi. Annual Review of Phytopathology 33: 369-391

Appel, DJ and Gordon TR (1995) Intra specific variation within populations of Fusarium oxysporum based on RFLP analysis of the intergenic spacer region of the CDNA. Experimental Mycology 19:120-128

Arganoza, MT, Ohrnberger J, Min J, Akins, RA (1994) Suppressor mutants of Neurospora crassa that tolerate allelic differences at single or at multiple heterokaryon incompatibility loci. Genetics $137(3)$ : 731-742

Asthana, A and Tuveson RW (1992) Effects of UV and phototoxins on selected fungal pathogens of citrus. Int.J.Plant Sce 153: 442-452 
Aylmore, RC and Todd, NK (1986) Cytology of non-self hyphal fusions and somatic incompatibility in Phanerochate velutina Journal of General Microbiology 132: 581-591

Boehm, EWA, Ploetz, RC, Kistler, HC (1994) Statistical analysis of electrophoretic karyotype variation among vegetative compatibility groups of Fusarium oxysporum f.sp. cubense. MPMI $7(2): 196-207$

Booth, C (1971) The genus Fusarium The Eastern Press Limited, London and Reading

Burnett, JH (1975) Mycogenetics John Wiley and Sons

Buxton, EW (1956) Heterokaryosis and parasexual

recombination in pathogenic strains of Fusarium

oxysporum. Journal of General Microbiology 15: 133-139

Buxton, EW (1962) Parasexual recombination in the bananawilt Fusarium. Trans. Brit. Soc. 45(2): 274-279

Buxton, EW and Ward V (1962) Genetic relationships between pathogenic strains of Fusarium oxysporum, Fusarium solani and an isolate of Nectria haematococca. Trans. Brit. Mycol. Soc. 45(2): 261-273

Carling, DE, Leiner, RH, Kebler KM (1987) Characterization of a new anastomosis group (AG-9) of Rhizoctonia solani. Phytopathology $77(11)$ : 1609-1612

Caten, CE and Jinks, JL (1966) Heterokaryosis: its significance in wild homothallic Ascomycetes and Fungi imperfecti. Transactions of the British Mycological Society 49: 81-93

Chacko, RJ, Weidemann, GJ, TeBeest, DO, Correl1, JC (1994) The use of vegetative compatibility and heterokaryosis to determine potential asexual gene exchange in colletotrichum gloeosporioides Biological Control 4 , $382-389$

Clutterbuck, AJ (1995) Chapter 11 in The growing fungus ed. by Gow, NAR and Gadd, GM, Chapman \& Hall, UK

Correll, JC, Puhalla, JE, Schneider RW (1986)

Identification of Fusarium oxysporum f.sp. apii on the basis of colony size, virulence and vegetative compatibility. Phytopathology 76(4): 396-400 
Correll, JC, Klittich, CJR, Leslie, JF (1987) Nitrate nonutilizing mutants of Fusarium oxysporum and their use in vegetative compatibility tests. Phytopathology 77: 1640-1646

Correll, JC (1991) The relationship between formae speciales, Races, and Vegetative Compatibility Groups in Fusarium oxysporum. Phytopathology 81(9): 1061-1064

Coenen, A, Debets, F, and Hoekstra, R (1994) Additive action of partial heterokaryon incompatibility (partial-het) genes in Aspergillus nidulans. Curr Genet $26: 233-237$

Collins, RA and Saville, BJ (1990) Independent transfer of mitochondrial chromosomes and plasmids during unstable vegetative fusion in Neurospora. Nature 345: 177-179

Cove, DJ (1976) Chlorate toxicity in Aspergillus nidulans: the selection and characterization of chlorate resistant mutants. Heredity 36: 191-203

Crawford MS, Chumley FG, Weaver, CG, Valent B (1986) Characterization of the heterokaryotic and vegetative hybrid phases of Magnaporthe grisea. Genetics 114: 1111-1129

Cubeta, MA, Briones-ortega, R Vilgalys, R (1993) Reassessment of heterokaryon formation in Rhizoctonia solani anastomosis group 4. Mycologia 85: 777-787

Daboussi, MJ, Gerlinger C (1992) Parasexual cycle and genetic analysis following protoplast fusion in Nectria haematococca. Current Genetics 21: 385-392

Debets, F, Yang, X, Griffiths, AJF (1994) Vegetative incompatibility in Neurospora: Its effect on horizontal transfer of mitochondrial plasmids and senescence in natural populations. Current Genetics 26: 114-119

Deleu, C, Clave, C, Begueret, J (1993) A single amino acid difference is sufficient to elicit vegetative incompatibility in the fungus Podospora anserina. Genetics 135: 45-52

Durand, N, Raymond, P, Fevre M (1992) Transmission and modification of transformation markers during an induced parasexual cycle in Penicillium roqueforti. Current Genetics 21: 377-383 
Elias, KS, Schneider, RW (1992) Genetic diversity within and among races and vegetative compatibility groups of Fusarium oxysporum fsp lycopersici as determined by isozyme analysis. Phytopathology 82(12): 1421-1427

Fincham, JRS, Day, PR, Radford, A (1979) Eungal Genetics University of California Press, Berkeley, Ca

Freitg, M, Didghde, N, Sachs, MS (1996) A UV induced mutation in Neurospora that affects translational regulation in response to arginine. Genetics 142: 117127

Gil, C, Pomes, R, Nombela, C (1988) A complementation analysis by parasexual recombination of Candida albicans morphological mutants Microb. 134: 1587-1595

Gokhal, DV (1992) Fungal protoplast fusion: A tool for breeding industrial strains. Journal of Scientific and Industrial Research 51(7): 497-506

Gordon, TR and okamoto, D (1992) Variation within and between populations of Fusarium oxysporum based on vegetative compatibility and mitochondrial DNA. Can J Bot 70: 1211-1217

Graeme-Cook, and Faull, JL Effect of ultraviolet-induced mutants of Trichoderma harzianum with altered antibiotic production on selected pathogens in vitro. Canadian Journal of Microbiology 37: 659-664

Gutz, H (1966) Induction of mitotic segregation with pfluoro-phenylalanine in Schizosaccharomyces pombe. Journal of Bacteriology 92(5): 1567-1568

Glass, NL and Kuldau, GA (1992) Mating type and vegetative incompatibility in filamentous ascomycetes. Annual Review of Phytopathology 30: 201-254

Glass, NL and Lee, L (1992) Isolation of Neurospora crassa $A$ mating type mutants by Repeat Induced Point (RIP) mutation. Genetics 132: 125-133

Hastie, AC (1970) The genetics of asexual phytopathogenic fungi with special reference to Verticillium. in Root Disease and Soil-Borne Pathogens, ed TA Toussoun, RV Bega and PE Nelson pp 55-62 Berkeley: University of California Press. 
Higgins, VJ, Miao V, Hollands J (1987) The use of Benomyl and cycloheximide resistance markers in studies of race development by the leaf mold pathogen Cladosporium fulvum. Canadian Journal of Plant Pathology 9: 14-19

Hocart, MJ, Lucas, JA, Peberdy, JF (1993) Characterization of the parasexual cycle in the eyespot fungus, Pseudocercosporella herpotrichoides. Mycological Research 97(8): 967-976

Hocart, MJ, McNaughton, JE (1994) Interspecific hybridization between Pseudocercosporella herpotrichoides and $P$. anguioides achieved through protoplast fusion. Mycol. Res. 98(1): 47-56

Holliday, $R$ (1956) A new method for the identification of biochemical mutants of microorganisms. Nature, Lond. 17: 987

Jackson, CW, Heale, JB (1987) Parasexual crosses by hyphal anastomosis and protoplast fusion in the entomopathogen Verticillium-lecani J Gen Microbiol 133(2): 3537-3548

Jacobson, DJ, Gordon, TR (1991) Fusarium oxysporum fsp melonis a case study of diversity within a forma specialis. Phytopathology 81(9): 1064-1067

Joaquim, TR and Rowe, RC (1990) Reassessment of Vegetative Compatibility Relationships among strains of Verticillium dahlieae using Nitrate Non-utilizing mutants. Phytopathology 80: 1160-1166

Kafer, E (1960) High frequency of spontaneous and induced somatic segregation in Aspergillus nidulans. Nature 186: $619-620$

Kistler, HC, Momol, EA, Benny, U (1991) Repetitive genomic sequences for determining relatedness among strains of Fusarium oxysporum. Phytopathology 81(3): 331-336

Kistler, HC and Miao, VPW (1992) New modes of genetic change in filamentous fungi. Annual Review of Phytopathology 30: 131-152

Klittich, CJR, Leslie, JF (1988) Nitrate reduction mutants of Fusarium moniliforme (Gibberella fujikuroi).

Genetics 118: 417-423 
L'Ogarro, LW and Clarkson, JM (1992) Variation for pathogenicity on tomato among parasexual recombinants of Verticillium dahliae. Plant Pathology 41: 141-147

Leslie, JF (1990) Genetic exchange within sexual and asexual populations of the genus Fusarium pp 37-48 in Fusarium Wilt of Banana ed. RC Ploetz, APS press, st. Paul, MN

Leslie, JF (1993) Fungal Vegetative Compatibility. Annual Review of Phytopathology 31: 127-150

Lhoas, P (1961) Mitotic haploidization by treatment of Aspergillus niger hybrids with p-fluorophenylalanine. Nature, London 190: 744

Mace, ME, Bell, AA, Beckman, CH (1981) Fungal Wilt Diseases of Plants Academic Press

Molnar, A, Sulyok, I, Hornok, L (1990) Parasexual recombination between vegetatively incompatible strains in Fusarium oxysporum. Mycol. Res. 94(3): 393-398

Nelson, PE, Toussoun TA, and Cook, RJ (1981) Eusarium: Diseases, Biology and Taxonomy The Pennsylvania state University Press, University Park and London

Newhouse Jr, MacDonald WL (1991) The ultrastructure of hyphal anastomoses between vegetatively compatible and incompatible virulent and hypovirulent strains of Cryphonectria-parasitica. Canadian Journal of Bot $69(3): 602-614$

Paccola-Meirelles, LD and Azevedo, JL (1991) Parasexuality in Beauvaria bassiana. Journal of Invertebrate Pathology 57: 172-176

Ploetz, RC (1990) Population biology of Fusarium oxysporum f.sp.cubense pp 63-76 in Fusarium wilt of Banana ed. RC Ploetz, APS press, st. Paul, MN

Ploetz, RC (1990) Variability in Fusarium oxysporum f.sp. cubense. Can. J. Bot 68: 1357-1363

Pontecorvo, GR, Roper, JA, Forbes E (1953) Genetic recombination without sexual reproduction in Aspergillus niger. J. Gen. Microbiology 8: 198-210 
Puhalla, JE (1985) Classification of strains of Fusarium oxysporum on the basis of vegetative compatibility. Can. J. Bot 63: 179-183

Puhalla, JE and Spieth, PT (1985) A comparison of heterokaryosis and vegetative incompatibility among varieties of Gibberella fujikoroi (Fusarium moniliforme). Experimental Mycology 9: 39-47

Puhalla, JE and Spieth, PT (1983) Heterokaryosis in Fusarium moniliforme Experimental Mycology 9: 39-47

Puhalla, JE and Mayfield, JE (1974) The mechanism of heterokaryotic growth in Verticillium dahliae. Genetics 76: $411-422$

Raper, JR (1966) Genetics of sexuality in Higher fungi New York:Ronald Press

Rayner, ADM (1991) The challenge of the individualistic mycelium. Mycologia 83(1): 48-71

Rizwana $R$ and Powell WA (1995) Ultraviolet Light-Induced heterokaryon formation and Parasexuality in cryphonectria parasitica. Experimental Mycology 19: 4860

Roper, JA and Pritchard, RH (1955) The recovery of complementary products of mitotic crossing over. Nature, London 175: 639

Sasaki, T (1992) Induction of ploidy level increments in an asporegenous industrial strain of the yeast Saccharomyces cerevisieae by UV irradiation. Applied and Environmental Microbiology 58(3): 948-952

Snyder, WC and Hansen HN (1954) Variation and speciation in the genus Fusarium Ann. NY Acad. Science 6016-23

Stover, RH (1990) Fusarium wilt of Banana: some history and current status of the disease. Chapter 1, page 7 in Fusarium wilt of Banana ed. RC Ploetz, APS press, st. Paul, MN

Symposium-Recent advances in Fusarium systematics (1990) Presented at the Annual Meeting of the American Phytopathological Society, Grand Rapids, Mi, August 7, 1990 
Volk, TJ (1989) Experimental studies on the morel.I. Heterokaryon formation between monoascosporous strains of Morchella. Mycologia 81: 523-531

Waite, B and Stover, RH (1960) Studies on Fusarium wilt of bananas VI.Variability and the cultivar concept in Fusarium oxysporum f.cubense Can. J. Bot. 38: 985-994

Walsh, TJ, Francesconi, A, Kasai, M and Chanock, SJ (1995) PCR and Single strand Conformational Polymorphism for recognition of medically important opportunistic fungi. Journal of Clinical Microbiology 33(12): 3216-3220

Wheelan, WL and Kwon-Chung KJ (1987) Parasexual genetics of Torulopsis glabrata. Journal of Bacteriology 196(11): 4991-4994

Wood, S and Kafer, E (1969) Effects of Ultraviolet irradiation on heterozygous diploids of Apergillus nidulans $I$. UV-Induced mitotic crossing over. Genetics $62: 507-518$ 
APPENDIXES

101 
Appendix A: Strains of Fusarium Oxysporum

I. Wild type strains of Fusarium oxysporum fsp cubense

\begin{tabular}{|c|c|c|c|}
\hline VCG & STRAIN & RACE & ORIGIN \\
\hline \multirow{5}{*}{0120} & STGM1 & 1 & Costa Rica \\
\hline & STC2 & 1 & Honduras \\
\hline & STH1 & 1 & Honduras \\
\hline & 22425 & 4 & Australia \\
\hline & SA8 & 4 & South Africa \\
\hline \multirow[t]{2}{*}{0121} & T3 & 4 & Taiwan \\
\hline & GM & 4 & Taiwan \\
\hline \multirow[t]{3}{*}{0122} & PH2 & 4 & Philippines \\
\hline & PW5 & 4 & Philippines \\
\hline & $S A B A$ & 2 & Philippines \\
\hline \multirow[t]{2}{*}{0123} & F9129 & 1 & Taiwan \\
\hline & DAVAO & 2 & Philippines \\
\hline \multirow[t]{4}{*}{0124} & MACA & 1 & Brazil \\
\hline & STD1 & 1 & Honduras \\
\hline & GMB & 1 & Brazil \\
\hline & JCB1 & 2 & Florida \\
\hline $0124 / 5$ & EA & ? & East Africa \\
\hline \multirow{2}{*}{0125} & 8610 & 1 & Australia \\
\hline & 8625 & 1 & Australia \\
\hline \multirow[t]{2}{*}{0126} & STB2 & 1 & Honduras \\
\hline & STM3 & 1 & Honduras \\
\hline \multirow[t]{2}{*}{0128} & A 47 & $?$ & Australia \\
\hline & 22994 & ? & Australia \\
\hline \multirow[t]{2}{*}{0129} & $0-1221$ & 1 & Australia \\
\hline & $N-5331$ & 4 & Australia \\
\hline \multirow[t]{2}{*}{1210} & F1 & $?$ & Cuba \\
\hline & $\mathrm{JC} 14$ & 1 & USA \\
\hline \multirow[t]{2}{*}{1211} & SH314222 & $?$ & $?$ \\
\hline & $1372-1$ & ? & Australia \\
\hline \multirow[t]{2}{*}{01212} & STNP-1 & $?$ & Tanzania \\
\hline & STNP-4 & $?$ & Tanzania \\
\hline & STSUM2 & $?$ & Indonesia \\
\hline \multirow{2}{*}{01214} & MW2 & $?$ & Malawi \\
\hline & MW4 1 & ? & Malawi \\
\hline
\end{tabular}

II. Formae speciales of Fusarium oxysporum

$\begin{array}{lll}\text { ISOLATE } & \text { RACE } & \text { FORMA SPECIALIS } \\ 73 & 1 & \text { lyCOpersici } \\ \text { FOLR1 } & 1 & \text { lyCopersici } \\ \text { FOLR3 } & 3 & \text { lyCOpersici } \\ 699 & & \text { raphani } \\ 777 & & \text { Conglutinans } \\ 247 & & \text { pisi }\end{array}$




\section{Appendix B: Media Preparation}

I. Complete media (CMA) (per liter)

Modified Czapeks dox: $20 \mathrm{~g}$ Dextrose, $3 \mathrm{~g}$ Yeast Extract (Difco), 3 g Peptone (Difco), 3 g Casein Hydrosylate (Difco), 2 g Sodium Nitrate $\left(\mathrm{NaNO}_{3}\right), .5 \mathrm{~g}$ Magnesium Sulfate $\left(\mathrm{MgSO}_{4}\right), .5 \mathrm{~g}$ Potassium Chloride (KCl), $20 \mathrm{~g}$ bacto-agar(Difco), $10 \mathrm{ml}$ Potassium Phosphate $\left(\mathrm{K}_{2} \mathrm{HPO}_{4}\right)$ $[.1 \mathrm{~g} / \mathrm{ml}]$ and $1 \mathrm{mI}$ Ferrous Sulfate $\left(\mathrm{FeSO}_{4}\right)[.01 \mathrm{~g} / \mathrm{ml}]$. CM top agar uses the same recipe except half the amount of the bacto-agar.

II. Minimum Media (MMA) (per liter)

$30 \mathrm{~g}$ Sucrose, $2 \mathrm{~g}$ Sodium Nitrate $\left(\mathrm{NaNO}_{3}\right), .5 \mathrm{~g}$ Magnesium Sulfate $\left(\mathrm{MgSO}_{4}\right), .5 \mathrm{~g}$ Potassium Chloride (KCl), 15 g bacto-agar (Difco), $10 \mathrm{ml}$ Potassium Phosphate [.1g/ml], 1 ml Ferrous Sulfate $\left(\mathrm{FeSO}_{4}\right)[.01 \mathrm{~g} / \mathrm{ml}], 100 \mu \mathrm{l}$ trace elements. MM top agar uses the same recipe except half the amount of the bacto-agar.

Liquid MM is as above without the addition of the bacto-agar.

III. Potato Dextrose Broth (PDB) (per liter)

$24 \mathrm{~g}$ Potato Dextrose Broth (Difco) in 1L distilled water.

IV. Benomyl stock solution (MMB/CMB) 
A stock solution was prepared by diluting $500 \mathrm{mg}$ of $50 \%$ benolate/benomyl (Dupont) in $100 \mathrm{ml}$ of chloroform for a final concentration of $5 \mathrm{mg} / \mathrm{ml}$. 


\section{Appendix C: Protocols}

\section{UV Mutagenesis}

\section{A. Generation of auxotrophic mutants}

Generation of auxotrophic mutants was achieved by inoculating $100 \mathrm{ml}$ of Potato Dextrose Broth (PDB) with a 2 $\mathrm{mm}^{2}$ square of filter stock of the wild type isolate we wanted to mutagenize. Growth was allowed for four days in a shaker at $180 \mathrm{rpm}$. The PDB solution was filtered into a sterile centrifuge tube using miracloth (Calbiochem) and a sterile funnel. The solution was then centrifuged for 10 minutes at $2000 \mathrm{rpm}$ and the supernatant was discarded. The remaining spore pellet was washed in $10 \mathrm{ml}$ sterile distilled water. The procedure was repeated twice. The final pellet was then resuspended in $10 \mathrm{ml}$ distilled water.

A spore count was done, using $10 u 1$ of the microconidia suspension (asexual spores) using the hemocytometer (Fisher Scientific). One million spores/ml, freshly harvested, were placed into a sterile $9 \mathrm{~cm}$ petri dish. $10 \mathrm{ml}$ distilled water was added to form a monolayer. The uncovered plate was then placed in a UV crosslinker (Fisher Scientific) and the microconidia were mutagenized at 20,000 micro joules/cm2 to achieve $90-95 \% \mathrm{kill}$.

Following the ultraviolet treatment, the spores were serially diluted and plated using $100 \mu \mathrm{l}$ of the desired 
dilution in $3 \mathrm{ml}$ CMA top agar to get 100 colonies/plate. Plates were stored in the dark to prevent photoreactivation. After 48 hours 1000 colonies were picked into a 50 square grid on a $9 \mathrm{~cm}$ MMA and CMA plate.

Auxotrophs grew on CMA but not on MMA. Putative auxotrophs were isolated and purified through a process of three single sporings.

The final stage on the isolation of mutants involves the preparation of a filter stock for preservation and storage. A Whatman filter No. 1 is placed on CMA and with a sterile toothpick, the purified auxotroph is picked on the the Whatman filter. Within a week, colony growth covers the filter. To insure even coverage, the filters are not lifted until mycelial growth is observed passed the filter paper. At that time, with sterile forceps, the filter is lifted and set to dry in a sterile, empty $9 \mathrm{~cm}$ petri dish. Once the filter was dried, with sterile scissors the filter was cut and stored in sterile containers in the refrigerator a $4^{\circ} \mathrm{C}$. To determine the nutritional deficiencies of the auxotroph, an auxanography test was performed using the Holliday method (Holliday 1956).

Double auxotrophs were made by collecting spores from single mutants grown in PDB and aerated in a shaker at $180 \mathrm{rpm}$ for four days. Microconidia suspension $\left(10^{6}\right.$ spores/ml) are re-mutagenized using the Fisher UV 
Crosslinker in the same way as with single mutants, to achieve 90-95\% kill. Serial dilutions of the spores after mutagenesis are plated on CMA. The emerging colonies are then tested on MMA supplemented with the auxotroph's first nutritional deficiency and CMA. Purification and storage of double auxotrophs are carried out in the same way single auxotrophs are.

Reversion tests were done on single and double auxotrophs. Cultures were grown in $200 \mathrm{ml}$ PDB shaking @ 180 rpm for four days. The spores are harvested by filtering through sterile miracloth (Calbiochem) to remove mycelial growth. The filtrate is centrifuged at $2000 \mathrm{rpm}$ for five minutes and the resulting pellet is washed with $10 \mathrm{ml}$ sterile distilled water. This procedure is repeated twice. The final spore pellet is resuspended in $1 \mathrm{ml}$ sterile distilled water and the number of spores determined using a hemocytometer (Fisher Scientific). The entire spore suspension is plated in MM top agar on a MMA plate. Kill curves were done to determine the optimum UV dose to achieve 95\% kill when searching for auxotrophs.

\section{B. The wild type testers: benomyl resistant mutants}

One million UV mutagenized spores/ml were plated on benomyl Complete Medium (CMB) plates at concentrations of 10, 50 and $100 \mu \mathrm{g} / \mathrm{ml}$ and allowed to grow $24 \mathrm{hrs}$ in the dark to prevent photoreactivation. Resistant colonies emerged 
approximately 1 week after plating and were transferred to fresh CMB plates to verify tolerance. The benomyl resistant auxotrophs were stored in filter paper (Whatman No.1) for further use.

II. Auxanography: The Holliday Test (Holliday 1956)

Analysis of auxotrophic mutants was made using a modified Holliday Test for bacterial auxotrophs. The composition of Media plates $1-5$ is listed vertically in the table. The composition of Media plates $6-10$ is listed horizontally. Medium 11 is an assortment of compounds not included in the others and is detailed horizontally at the end of the table.

1

6 adenosine

7 histidine

8 phenylalanine tyrosine

9 glutamine

10 thymine asparagine uracil serine

glutamate

DAP

4 5

11 pyridoxin, nicotinic acid, biotin, pantothenate, alanine 
Notes:

1. Thymine needs to be heated to $-65^{\circ} \mathrm{C}$ before adding it to the mixture.

2. Guanosine needs to be heated to $-85^{\circ} \mathrm{C}$ before adding it to the mixture.

3. Some purine mutants grow on adenosine or guanosine; they will grow on pools $1,2 \& 6$.

4. Some purine mutants require adenosine + thymine; they will grow only on pool 6 .

5. PyrA mutants require uracil + arginine; they grow on pool 9 .

6. Mutants requiring Isoleucine + valine will grow only on pool 7 .

7. Mutants with early blocks in the aromatic pathway will only grow on pool 8 .

8. Early blocks in the lysine pathway grow only on pool 4 .

9. Use salts of glutamic and aspartic acids

10. Keep solutions containing trp in the dark. 
Nutritional supplements

stocks solutions are such that $5 \mathrm{ml}$ of the solution added to

1 liter of medium will result in the appropriate

concentration $(200 \mathrm{x})$

Nutrient

Adenosine

Alanine

Arginine

Asparagine

Aspartate-K

Biotin

Citrulline

Cysteine

Cystathionine

DAP

Glutamate-Na

Glutamine

Glycine

Guanosine

Histidine

Homoserine

Isoleucine

Leucine

Lysine

Methionine

Nicotinic acid

ornithine

Pantothenate-Ca

Phenylalanine

Proline

Pyridoxine-HCl

serine

Thiamine

Threonine

Thymine

Tryptophan

Tyrosine

Uracil

Valine stock sol.

2.67

0.84

2.53

0.84

1.00

0.49

2.00

0.73

0.80

0.38

5.00

14.6

0.20

1.70

0.31

0.06

0.79

0.79

1.10

0.90

0.25

2.00

0.48

0.99

4.60

0.41

8.40

0.337

0.71

0.81

0.41

0.36

0.224

0.70
(\%) Sterilization Remarks

$A$
$A$
$A$
$F$
$F$
$A$
$A$
$F$

$\mathrm{A}$ in $1 \mathrm{~N} \mathrm{HCl}$

A

F

A

A

A

A

A

A

A

A

A

A

A

A

A

A

A

A

A

A

A

A

A

A

A 
Due to the high incidence of arginine and methionine auxotrophic mutants, further analysis was done to identify complementation groups within the pool of Arg and Met auxotrophic mutants (Finchmann 1979, Burnett 1975). Metabolic Pathway - Methionine

\begin{tabular}{||l|l|l|l|l|}
\hline GROUP & CYSTEINE & CYSTATHIONINE & HOMOSERINE & METHIONINE \\
\hline Met1 & - & - & - & + \\
\hline Met2 & - & - & + & + \\
\hline Met3 & - & + & + & + \\
\hline Met4 & + & + & + & + \\
\hline
\end{tabular}

Metabolic Pathway - Arginine

\begin{tabular}{||l|l|l|l|l||}
\hline GROUP & ORNITHINE & CITRULLINE & PROLINE & ARGININE \\
\hline Arg1 & + & + & - & + \\
\hline Arg2 & - & + & - & + \\
\hline Arg3 & - & - & - & + \\
\hline Arg4 & + & + & + & + \\
\hline
\end{tabular}


IV.

\section{Heterokaryon Formation}

\section{A. Pairings}

\section{The Double Pick Method}

In the double-pick method inoculum of one parent was transferred to a MMA plate by picking with a sterile toothpick at the edge of the colony. With a different sterile toothpick the second parent was picked in the same fashion and co-inoculated into the same hole as parent one. Both the controls and the pairings are picked in the same plate. Heterokaryon formation is seen in 72 hours. To determine the number of spores present in a single pick I washed the toothpick in $1 \mathrm{ml}$ sterile distilled water. With a hemocytometer (Fisher scientific) it was determined that there were $10^{3}-10^{5}$ spores $/ \mathrm{ml}$. Serial dilutions of the spore suspension were plated on CMA. The cfus recovered were representative of the number of spores plated.

\section{Block}

Two by two $\mathrm{mm}^{2}$ blocks are cut from the edge of growing colonies of each parental phenotype on CMA. Each block is then placed face-to-face on MMA. As a control, blocks of each "parent" are paired with themselves on MMA. Growth onto the MMA is further analyzed for heterokaryon formation.

\section{Liquid Minimal Media}


Equal number of spores from each "parent" is co-inoculated in $1 \mathrm{ml}$ of liquid MM in a $13 \mathrm{x} 100 \mathrm{~mm}$ test tube. The test tubes are placed at an angle to insure proper aeration in a shaker at $180 \mathrm{rpm}$ at room temperature for four days. Control tubes contain spores from the single parents. Serial dilutions of this suspension are then plated in MM top agar onto MMA. Colonies that grow on MMA are further analyzed for het formation.

\section{B. Cellophane Test}

Auxotrophs might be able to complement each other on MMA by cross feeding rather than hyphal anastomosis. To rule out cross feeding in our pairings we performed each method under conditions that would allow cross feeding but prevented anastomosis, modifying the technique used by Correll (1986).

\section{Double-pick Method}

A strip of sterile cellophane was held vertically in a petri dish by MMA. Each parental strain was picked on opposite sides of the cellophane strip. As control, we double-picked the parents in the same hole without the cellophane. No growth was observed when cellophane separated the picks. 


\section{Block}

A strip of sterile cellophane was placed between two blocks of mycelia paired on MMA, representing both parental phenotypes. No growth was detected in the area between the blocks.

\section{Liquid Minimal Media}

A dialysis bag containing a spore suspension in LMM of one parent was submerged in a sterile $50 \mathrm{ml}$ centrifuge tube (Corning) in which spores from the second parent were suspended in liquid MM. As control, a microconidia suspension representing both "parents" was mixed together in another tube. No growth was detected in the tube containing the dialysis bag. A mycelial plug formed in the control tube.

v. Diagnostic Test to screen microconidia

To determine the phenotype of a putative heterokaryon the microconidia are washed with $10 \mathrm{ml}$ sterile liquid MM and loosened with a sterile spreader, collected in a sterile 50 mI centrifuge tube (Corning) and counted with a hemocytometer (Fisher) by placing $10 \mu l$ of a dilution in each end of the hemocytometer. A grid in the hemocytometer consists of 25 squares separated by triple lines. Spores are counted on the four corner squares and in the middle one. The number of spores is then multiplied by $50 \times 10^{3} \mathrm{x}$ 
dilution. Using the spore count as a reference, serial dilutions of the spore suspension are plated, looking for a dilution that would yield around 100 colonies/plate. Serial dilutions are made as follows: from the spore stock, $100 \mu 1$ of the spores are diluted in $900 \mu l$ of sterile distilled water for a total volume of $1 \mathrm{ml}$. $100 \mu \mathrm{l}$ of the diluted spores are further transferred to $900 \mu l$ of sterile distilled water. The procedure is repeated until the desired dilution is achieved.

An aliquot of $100 \mu \mathrm{l}$ from the last dilution tube is then transferred to a sterile test tube. The spores are then overlaid with $3 \mathrm{ml} \mathrm{CM}$ Top Agar and are plated in a $9 \mathrm{~cm}$ CMA petri dish to form a monolayer, thus ensuring even distribution of the spores on the plate. The emerging colony forming units (cfus) are then tested in diagnostic plates. With a sterile toothpick, the edge of the colony is picked and transferred to each of four plates, MMA, MMA supplemented with the amino acid deficiencies of either parental phenotype and CMA as control. The growth of the cfus is scored in each plate and the phenotype of the tested colony is assessed. 
A. Expected Outcome: Heterokaryon Formation Using Complementary Auxotrophic Mutants

\begin{tabular}{||l|l|}
\hline $\begin{array}{l}\text { PHENOTYPES: } \\
\text { (auXotrophs) }\end{array}$ & $\begin{array}{l}\text { MINIMAL } \\
\text { MEDIA (MMA) }\end{array}$ \\
\hline PARENT 1 (P1) & - \\
\hline PARENT 2 (P2) & - \\
\hline HETEROKARYON & + \\
\hline
\end{tabular}

\section{B. Analysis of Microconidia from Complementary Heterokaryons}

\begin{tabular}{|l|l|l|l|l|}
\hline $\begin{array}{l}\text { PHENOTYPES: } \\
\text { (auxotrophs) }\end{array}$ & (MMA) & $\begin{array}{l}\text { MMA + P1 } \\
\text { SUPPLEMENT }\end{array}$ & $\begin{array}{l}\text { MMA + P2 } \\
\text { SUPPLEMENT }\end{array}$ & (CMA) \\
\hline PARENT 1 (P1) & - & + & - & + \\
\hline PARENT 2 (P2) & - & - & + & + \\
\hline HET/Hybrid & + & + & + & + \\
\hline
\end{tabular}

c. Expected Outcome: Heterokaryon Formation Using wild Type Testers

\begin{tabular}{||l|l|}
\hline PHENOTYPES: & $\begin{array}{l}\text { MINIMAL } \\
\text { MEDIA + BEN } \\
\text { (MMB) }\end{array}$ \\
\hline $\begin{array}{l}\text { WILD TYPE TESTER } \\
\text { (WTT) }\end{array}$ & - \\
\hline WILD TYPE & - \\
\hline HETEROKARYON & + \\
\hline
\end{tabular}

D. Analysis of Microconidia from Heterokaryons Using Wild Type Testers

\begin{tabular}{|l|l|l|l|l|}
\hline & $\begin{array}{l}\text { MINIMAL } \\
\text { MEDIA(MMA) }\end{array}$ & $\begin{array}{l}\text { MMA + } \\
\text { BENOMYL }\end{array}$ & $\begin{array}{l}\text { COMPLETE } \\
\text { MEDIA(CMA) }\end{array}$ & $\begin{array}{l}\text { CMA + } \\
\text { BENOMY }\end{array}$ \\
\hline WILD TYPE (WT) & + & - & + & - \\
\hline $\begin{array}{l}\text { WILD TYPE TESTER } \\
\text { (WTT) }\end{array}$ & - & - & + & + \\
\hline $\begin{array}{l}\text { HETEROKARYON } \\
\text { (WT X WTT) }\end{array}$ & + & + & + & + \\
\hline
\end{tabular}


VI. Hybrid Enhancement

Equal number of freshly harvested spores from each auxotrophic parent are mixed together in $5 \mathrm{ml}$ Liquid Minimal Medium (LMM) in a sterile $13 \times 100 \mathrm{~mm}$ capped test tube. The tubes are placed in a shaker at $180 \mathrm{rpm} \times 2$ weeks. As control, equal number of spores from each parental phenotype are individually inoculated into $5 \mathrm{ml}$ Liquid MM and placed in a shaker at $180 \mathrm{rpm} \times 2$ weeks.

At the end of two weeks any mycelial growth present is filtered out and the filtered spores are plated at high and low density in MMA and at low density in CMA as control. Prototrophic growth in MMA at low density is indicative of putative hybrid formation. The putative hybrids are transferred to fresh MMA plates and screened for continued growth. After several successful transfers the hybrid is stored by placing a whatman No. 1 filter in MMA and inoculating the filter by picking from the edge of a hybrid into the filter.

The original mycelial plug is transferred to MMA for further analysis (See diagnostic test). Spores from colonies showing hybrid phenotypes are collected and plated at low density on CMA plates. Individual colonies are screened using the diagnostic test to determine the phenotype. As control, spores from each individual parent were placed in the shaker under similar conditions. Hybrids 
are never removed from selective pressure unless screening for haploidization and/or segregation, at which time they are grown on CMA.

VII. Hyphal Tipping

Plugs from putative hybrids are transferred to $1.5 \%$ water agar plates poured thinly ( $4 \mathrm{ml} / \mathrm{plate})$ to allow for sparse growth. Using platinum needles under a dissecting microscope, the hyphal tips of growing mycelia are removed and transferred to either complete media or minimal media plates. Spores are collected from viable tips and are screened on diagnostic plates to assess their phenotype. 
Appendix D: Auxotrophic Mutants of FOC 


\begin{tabular}{|c|c|c|c|c|c|c|}
\hline $\begin{array}{l}\text { STRAIN } \\
120204\end{array}$ & $\begin{array}{l}\text { VCG } \\
120\end{array}$ & $\begin{array}{l}\text { RACE } \\
4\end{array}$ & $\begin{array}{l}\text { MUTANT } \\
\text { SA8 } 697\end{array}$ & $\begin{array}{l}\text { AUX1 } \\
A D E\end{array}$ & AUX2 & $A \cup \times 3$ \\
\hline 124303 & 124 & 1 & GMB 604 & $A D E$ & & \\
\hline 124306 & 124 & 1 & GMB 258 & ADE & & \\
\hline 120504 & 120 & 4 & 22425335 & ARG & & \\
\hline 120313 & 120 & 1 & STC2 241 & ARG-1 & & \\
\hline 120401 & 120 & 1 & STGM1 & ARG-2 & & \\
\hline 120405 & 120 & 1 & STGM1 4OR & ARG-2 & & \\
\hline 120411 & 120 & 1 & STGM1 501 & ARG-2 & & \\
\hline 120412 & 120 & 1 & STGM1 505 & ARG-2 & & \\
\hline 120413 & 120 & 1 & STGM1 506 & ARG-2 & & \\
\hline 120414 & 120 & 1 & STGM1 507 & ARG-2 & & \\
\hline 120415 & 120 & 1 & STGM1 508 & ARG-2 & & \\
\hline 120416 & 120 & 1 & STGM1 509 & ARG-2 & & \\
\hline 120417 & 120 & 1 & STGM1 512 & ARG-2 & & \\
\hline 120418 & 120 & 1 & STGM1 514 & ARG-2 & & \\
\hline 120419 & 120 & 1 & STGM1 515 & ARG-2 & & \\
\hline 120420 & 120 & 1 & STGM1 516 & ARG-2 & & \\
\hline 120421 & 120 & 1 & STGM1 518 & ARG-2 & & \\
\hline 120422 & 120 & 1 & STGM1 519 & ARG-2 & & \\
\hline 120423 & 120 & 1 & STGM1 520 & ARG-2 & & \\
\hline 120424 & 120 & 1 & STGM1 521 & ARG-2 & & \\
\hline 120425 & 120 & 1 & STGM1 522 & ARG-2 & & \\
\hline 120426 & 120 & 1 & STGM1 502 & ARG-2 & & \\
\hline 120432 & 120 & 1 & STGM1 18 & ARG-2 & & \\
\hline 123201 & 123 & 1 & F9129-332 & ARG-2 & & \\
\hline 124202 & 124 & & MACA & ARG-2 & & \\
\hline 124313 & 124 & 1 & GMB FE 580 & ARG-2 & & \\
\hline 124503 & 124 & & JCB1 139 & ARG-2 & & \\
\hline 126106 & 126 & 1 & STM3 107 & ARG-2 & & \\
\hline 120430 & 120 & 1 & STGM1P & ARG-2 & ADE & \\
\hline 120431 & 120 & 1 & STGM1 22 & ARG-2 & ADE & \\
\hline 120402 & 120 & 1 & STGM1 124 & ARG-2 & $A D E-1$ & \\
\hline 120427 & 120 & 1 & STGM1 39 & ARG-2 & MET & \\
\hline 120428 & 120 & 1 & STGM1 470 & ARG-2 & MET-4 & \\
\hline 120429 & 120 & 1 & STGM1 32 & ARG-2 & MET-4 & \\
\hline 120303 & 120 & 1 & STC2 349 & ARG-3 & & \\
\hline 120305 & 120 & 1 & STC2 228 & ARG-3 & & \\
\hline 120503 & 120 & 4 & 22425330 & ARG-3 & & \\
\hline 120601 & 120 & 1 & STH1 ARG-3 & & & \\
\hline 124312 & 124 & 1 & GMB FE 701 & ARG-3 & & \\
\hline 124601 & 124 & 1 & STD1 ARG-3 & & & \\
\hline 124521002 & 124 & & EA25 105 & ARG-3 & & \\
\hline 120320 & 120 & 1 & STC2 609 & ARG-3 & $A D E$ & \\
\hline 120602 & 120 & 1 & STH1 401 & ARG-3 & $A D E-2$ & \\
\hline 120603 & 120 & 1 & STH1 401B & ARG-3 & ADE-2 & $B E N+$ \\
\hline 120507 & 120 & 4 & $22425330 \mathrm{~B}$ & ARG-3 & $\mathrm{BEN}+$ & \\
\hline 120513 & 120 & 4 & $22425-86$ & ARG-3 & BEN+ & LYS \\
\hline 120511 & 120 & 4 & 22425330 & ARG-3 & ILE & \\
\hline 124602 & 124 & 1 & STD1 114 & ARG-3 & MET-4 & \\
\hline 124519001 & 124 & & EA23 & $\mathrm{BEN}+$ & & \\
\hline 120410 & 120 & 1 & STGM1 & $\mathrm{BEN}+$ & & \\
\hline
\end{tabular}




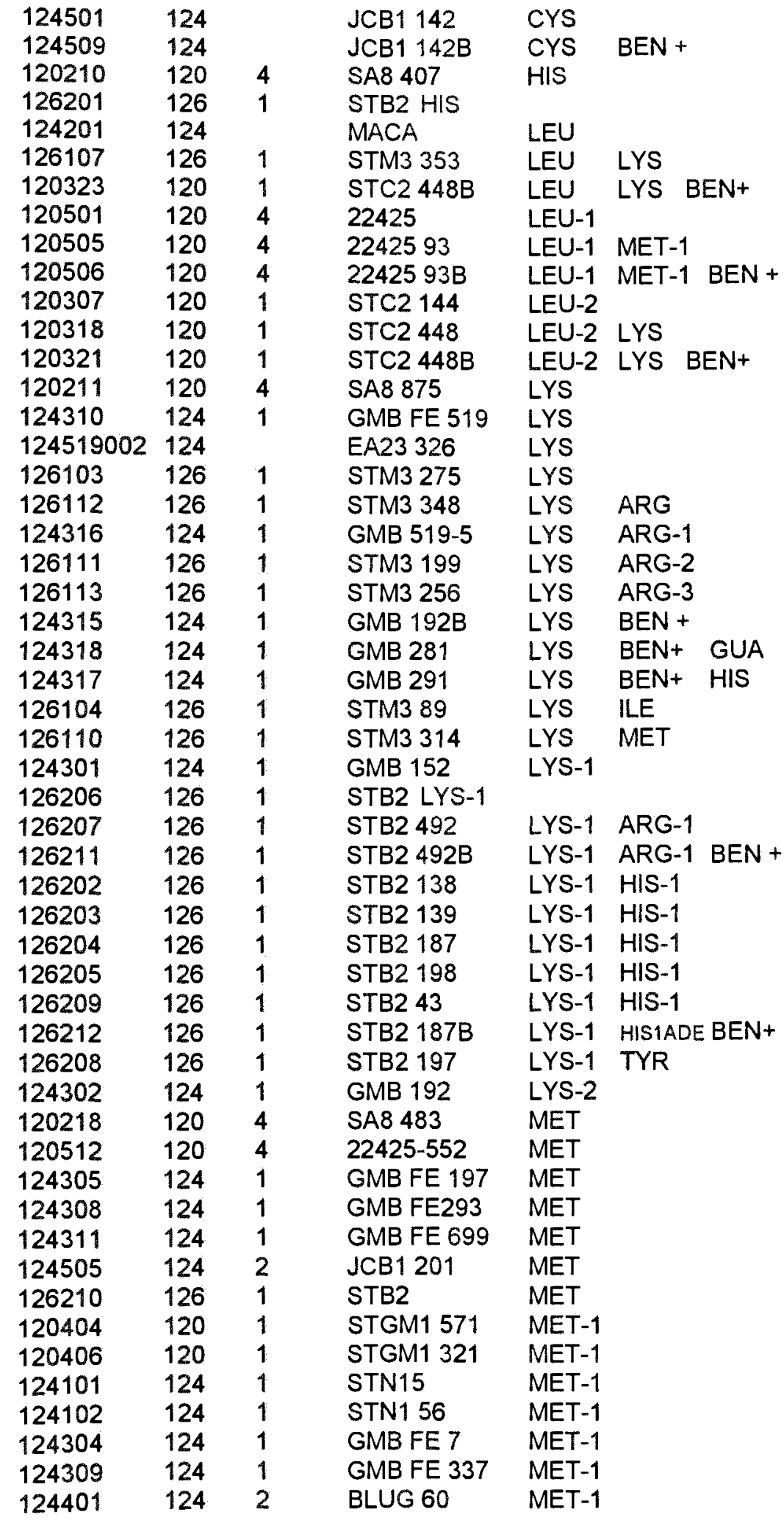




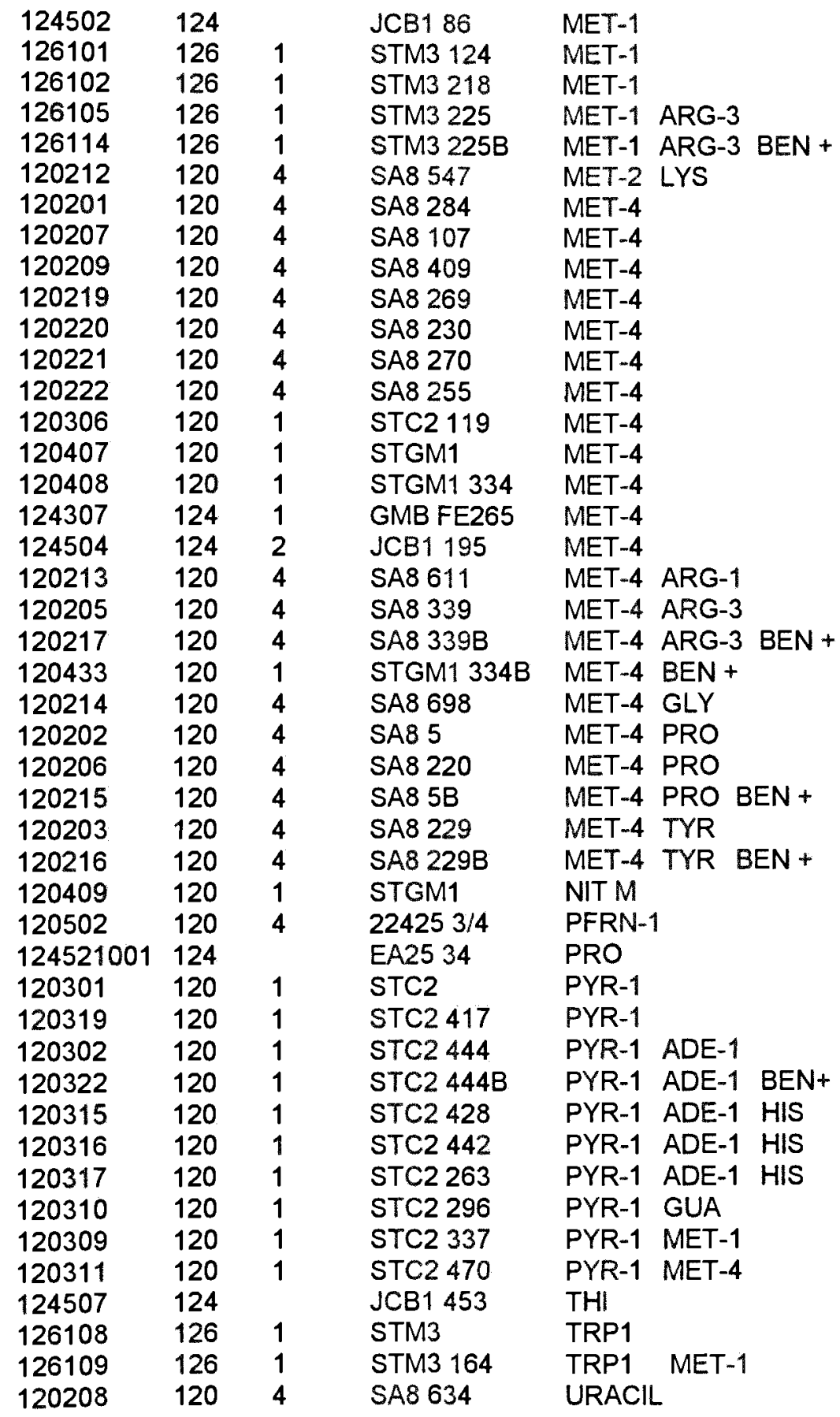


II. Distribution of auxotrophic mutants by VCG

\begin{tabular}{|c|c|c|c|c|}
\hline VCG & RACE & STRAIN & $\mathrm{AMT}$ & AUXOTROPHY \\
\hline \multirow[t]{5}{*}{0120} & 1 & STGM1 & 25 & $\operatorname{Arg} 2(20), \operatorname{Arg} 2 \operatorname{Ade} 1$ (1) Met1 (2), Met4 (2) \\
\hline & 1 & STH1 & 2 & Arg3 (1), Arg3 Ade1 (1) \\
\hline & 1 & STC2 & 14 & $\begin{array}{l}\text { Arg3 (2), Leu (1), Met4 (1), Pyr1 (2), Pyr Ade1 (1), } \\
\text { Pyr1 Ade His (3), Pyr1 Gua (1), Pyr1 Lys (1), Pyr1 } \\
\text { Met-1 (1), Pyr1 Met4 (1) }\end{array}$ \\
\hline & 4 & 22425 & 4 & $\operatorname{Arg} 3(2), \operatorname{Arg} 3 \operatorname{ILE}(1)$, Leu (1), Leu Met1 (1) \\
\hline & & SA8 & 14 & $\begin{array}{l}\text { Ade1 (1), His (1), Lys (1), Met2 Lys (1), Met4 (3), } \\
\text { Met4 Arg1 (1), Met4 Arg3 (1) Met4 Gly (1), Met4 Pro } \\
\text { (2), Met4 Tyr (1), Uracil (1) }\end{array}$ \\
\hline 0123 & 1 & F9129 & 1 & $\operatorname{Arg2}(1)$ \\
\hline \multirow[t]{6}{*}{0124} & 1 & GMB & 14 & $\begin{array}{l}\text { Ade1 (1), Ade2 (1), Arg2 (1), Arg3 (1), Lys (3), Lys } \\
\text { Arg 1 (1), Met (3) Met1 (2), Met4 (1) }\end{array}$ \\
\hline & 1 & MACA & 2 & $\operatorname{Arg} 2(1)$, Leu $(1)$ \\
\hline & 1 & STD1 & 2 & $\operatorname{Arg} 3(1), \operatorname{Arg} 3 \operatorname{Met} 4(1)$ \\
\hline & 2 & BLUG & 1 & Met1 (1) \\
\hline & 2 & JCB1 & 6 & $\operatorname{Arg} 2(1), \operatorname{Cys}(1), \operatorname{Met} 4(2), \operatorname{Met} 1(1), \operatorname{Thi}(1)$ \\
\hline & 2 & STN1 & 2 & Met1 (2) \\
\hline \multirow[t]{2}{*}{$0124 / 5$} & & EA23 & 1 & Lys (1) \\
\hline & & EA25 & 2 & $\operatorname{Arg} 3(1), \operatorname{Pro}(1)$ \\
\hline \multirow[t]{2}{*}{0126} & 1 & STB2 & 10 & $\begin{array}{l}\text { His (1), Lys1 (1), Lys1 Arg1 (1), Lys1 His (5), Met } \\
\text { (1) Lys Tyr (1) }\end{array}$ \\
\hline & 1 & STM3 & 11 & $\begin{array}{l}\text { Arg2 (1), Leu Lys (1), Lys Arg3 (3), Lys Ile (1), Lys } \\
\text { Met (1), Met1 (1), Met1 Arg-3 (1), Trp (1), TRP Met } \\
\text { (1) }\end{array}$ \\
\hline
\end{tabular}




\section{DISTRIBUTION OF AUXOTROPHIC MUTANTS BY VCG}

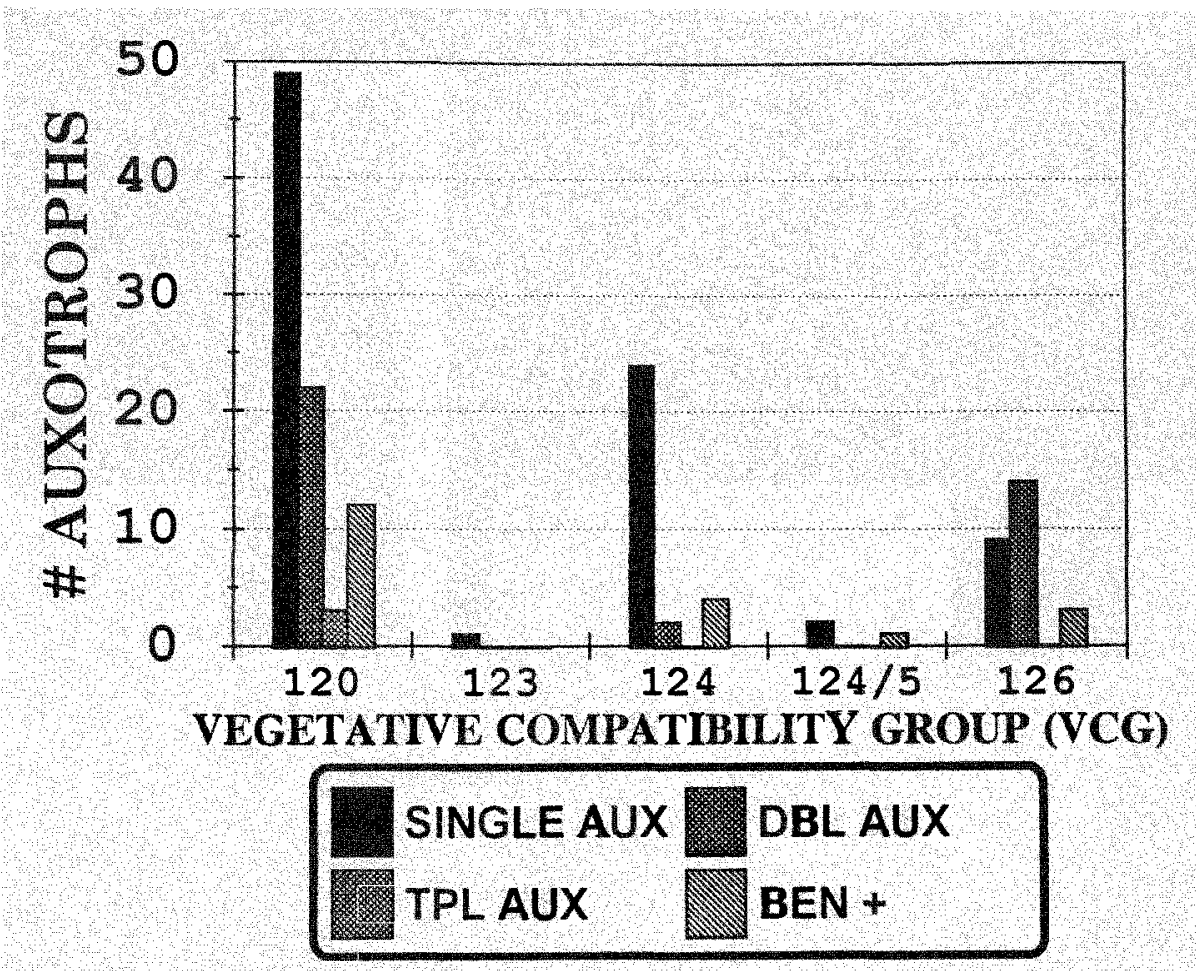

Figure 25 Distribution of auxotrophic mutants by VCG. The first bar represents single auxotrophs, the second bar, double auxotrophs, the third bar, triple auxotrophs and the last bar represents benomyl-resistant wild type testers. 
Appendix E: Pairings Between Auxotrophic Mutants of FoC (The Grid) 







